
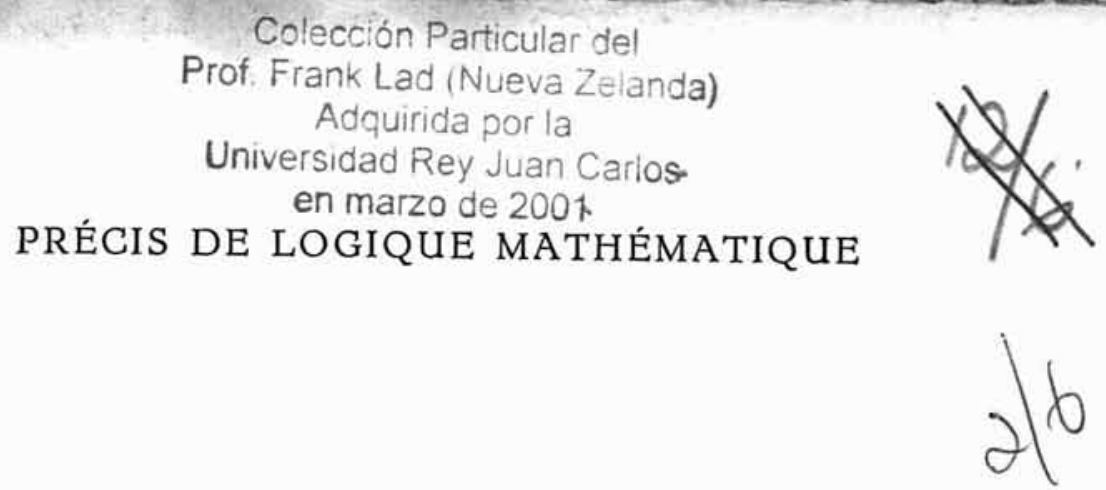

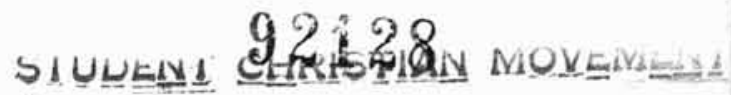

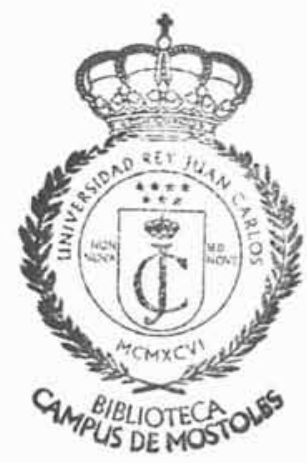




\section{P. Runmill. itt champion St. St. Albans COLLECTION SYNTHESE Chrintehuret. 2}

\section{PRÉCIS DE LOGIQUE MATHÉMATIQUE}

PAR

I. M. BOCHEŃSKI O.P.

Professeur à l'Université de Fribourg

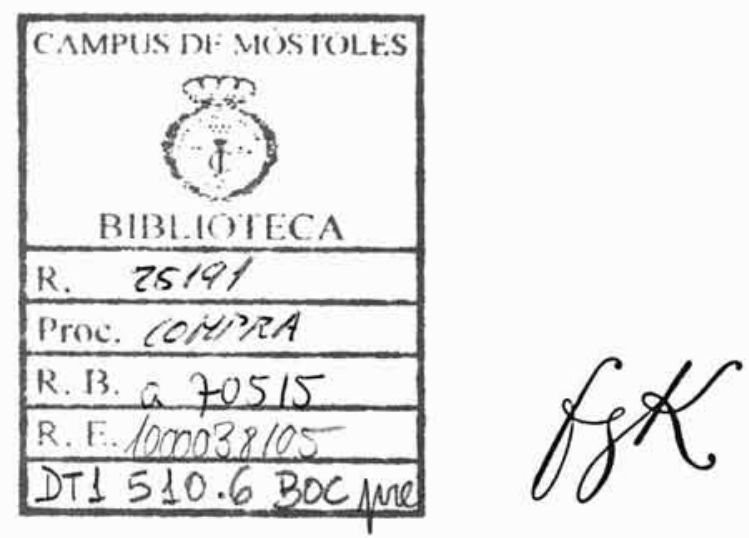

F. G. KROONDER/BUSSUM/PAYS-BAS 


\section{PRÉFACE}

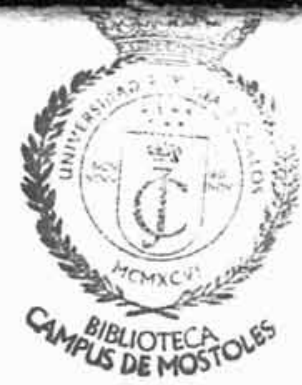

On se propose dans ce précis un double but: (1) fournir un texte qui pourrait servir de base à l'enseignement oral élémentaire, (2) résumer les lois les plus utiles aux applications non-mathématiques de la logique.

Un développement relativement considérable a été donné à la théorie des propositions, et ceci non seulement à cause de l'importance fondamentale de ce traité, mais aussi pour faciliter au lecteur, sur un exemple concret, la compréhension des méthodes les plus rigoureuses de la logique contemporaine. Les autres traités ont été, par contre, exposés d'une manière plus sommaire et moins rigoureuse. Le contenu du précis correspond à peu près à celui de 1 ',Abriss der Logistik" de Rudolf Carnap; il comprend cependant un nombre plus considérable de lois (plusieurs centaines); il donne à côté du symbolisme péanorussellien celui de Łukasiewicz et contient aussi certains résultats des recherches postérieures à la publication du travail mentionné.

Le précis n'est pas une traduction, ni même une refonte des „Nove lezioni di logica simbolica" publiées en 1938. Les thèses centrales en sont les mêmes; l'auteur a cependant changé d'opinion sur certains points secondaires; il donne aussi dans le présent ouvrage une matière beaucoup plus ample qu'auparavant.

Tout problème philosophique a été délibérément écarté; on a évité même d'entrer dans les discussions proprement logiques, concernant par exemple le principe des indiscernables ou la théorie des types.

L'auteur tient à remercier M E. W. BETH, professeur à l'Université d'Amsterdam, qui a bien voulu lire le manuscrit; il lui doit, en dehors de plusieurs suggestions et corrections, l'encouragement à publier ce précis. Il remercie aussi le Comité de Rédaction de la Collection Synthese et l'Editeur, M F. G. KROONDER, qui ont rendu la publication possible.

Ce petit manuel doit cependant le plus aux logiciens polonais: il est conçu dans l'esprit qu'ils ont créé et il utilise en partie leurs résultats. Il est dédié à la mémoire de ceux d'entre eux qui trouvèrent la mort de mains barbares dans les camps de concentration, au ghetto de Varsovie et pendant l'insurrection de 1944. 


\section{GÉNÉRALITÉS}

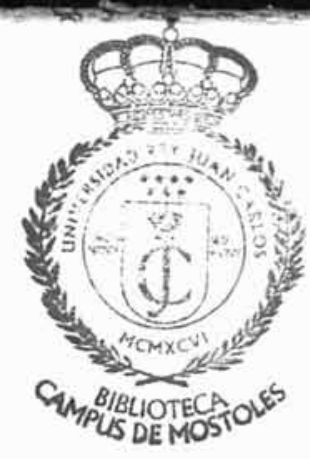

$\S 0$. Introduction. 0.1. Notion et histoire. La logique mathématique, dite aussi "logistique", ,logique symbolique”, „logique algébrique” et, plus récemment, „logique formelle" tout court, est l'ensemble des théories logiques élaborées au cours du dernier siècle à l'aide d'un symbolisme conventionnel et d'une méthode déductive rigoureuse. Leibniz (1646-1716) est généralement reconnu comme premier logicien mathématique; mais ce furent George Boole (1815-1864) et Augustus de Morgan (18061871) qui les premiers donnèrent (1847) des systèmes pareils dans leur forme à ceux d'aujourd'hui. Leurs travaux furent repris et approfondis par Gottlob Frege (1848-1925) et Giuseppe Peano (18581932), puis par Alfred North Whitehead et Bertrand Russell dans leur oeuvre monumentale „Principia Mathematica" (1910-1913). Depuis, des écoles actives de logique mathématique ont surgi dans de nombreux pays, surtout en Amérique, en Allemagne et en Pologne; le progrès fut très rapide; il continue encore aujourd'hui.

0.2. Logique et mathématiques. La logique mathématique est dite "mathématique" à cause de son origine, car elle a été développée surtout dans le but d'examiner les fondements de cette science. Il y a d'ailleurs une certaine ressemblance extérieure entre l'aspect de ses formules et celui des formules mathématiques. Certains logiciens prétendent aussi que les mathématiques ne sont qu'une partie de la logique; mais cette opinion est loin d'être généralement reçue. Hormis cela, il n'y a rien de mathématique dans la logique mathématique: elle ne traite ni des nombres ni des quantités, mais d'objets quelconques.

0.3. Applications. La logique mathématique a été appliquée avec succès non seulement aux mathématiques, mais aussi à la physique (R. Carnap, A. Dittrich, B. Russell, C. E. Shannon, A. N. Whitehead, H. Reichenbach, P. Destouches), à la biologie (J. H. Woodger et A. Tarski), à la psychologie (F. B. Fitsch), aux questions pratiques (E. C. Berkeley, E. Stamm), au droit et à la morale (K. Menger), à la métaphysique elle-même (J. Salamucha, H. Scholz et l'auteur). Très 
fécondes ont été les applications à l'histoire de la logique (J. Łukasiewicz, H. Scholz, A. Becker, J. Salamucha, K. Dürr, Z. Jordan, P. Boehner et l'auteur); en particulier J. Łukasiewicz, J. Salamucha et d'autres ont montré à l'aide des méthodes mathématico-logiques que toute l'époque moderne méconnaissait le vrai sens de nombreux textes d'Aristote, de presque toute la logique stoïcienne, d'à peu près toute celle des Scolastiques. Il existe enfin des applications à la théologie catholique (F. Drewnowski, J. Salamucha). Cependant il semble qu'on n'est qu'au commencement. Il paraît certain que, d'une part, même la logique déjà élaborée n'a été utilisée qu'en petite partie et que, d'autre part, un développement très considérable des théories existantes est possible et est en train de s'accomplir.

Histolre: L'histoire de la logique formelle est une science récente: J. Eukasiewicz (1921) et H. Scholz (1931) en sont les principaux novateurs. - Les discussions sur les relations entre la logique et les mathématiques datent de Leibniz, qui conçut l'idée d'une „mathesis universalis"; le problème fut posé dans toute son acuité par Peano. - Les applications et les discussions philosophiques de la logique moderne datent aussi toutes du XXe siècle.

LitTÉRATURE: Pour l'histoire de la logique: Eukasiewicz, Zur Geschichte; Scholz, Geschichte; Beth, Geschiedenis. (b) Logique et mathématiques: Gonseth, Les entretiens (donne un aperçu sur l'état actuel des problèmes); PM; Russell, Introduction (pour la déducibilité); Heyting, Die intuitionistische Mathematik, (contre). (c) Logique mathématique et logique traditionnelle: Lewis, A survey (pour l'opposition); Greenwood, Les fondements (contre). (d) Introductions: Carnap, Abriss; Hilbert und Ackermann, Grundzüge; A. Tarski, Introduction; (tous les trois excellents). Il n'y a pas d'introduction satisfaisante en français: Boll, Manuel, représente un niveau déjà dépassé, (e) Traités: PM (dépassé en de nombreux points, mais reste toujours le répertoire le plus complet); Hilbert und Berneys, Grundlagen der Math.; Quine, Mathematical Logic; Feys, Logistiek, I. (les deux derniers à recommander).

BIBLtogRAPHIE: Church, A Bibliography (complète pour la période de 1666-1935 inclusivement; elle se continue dans le JSL). Beth, Mathematische Logik (excellente bibliographie méthodiquement sélectionée). Cf. les travaux de Quine et Feys précites.

$\S$ 1. EXPRESSIONS ET OPÉRATIONS

Le but de ce chapitre est d'énumérer FONDAMENTALES.

les noms des expressions fondamentales de la logique mathématique, d'expliquer leur sens, sans les définir d'une manière stricte, et de décrire quelques opérations fondamentales pour la logique. Toute la théorie présente porte sur les noms des expressions, non pas sur les expressions elles-mêmes (cf. 2.1). C'est donc une théorie métalogique (cf. 2.16). 


\subsection{Expression, constante, variable.}

1.11. „Expression" - ,signe graphique ou groupe de signes graphiques".

1.12. "Expression du système $S "$ — ,expression formée conformément aux règles du système $S^{\prime \prime}$.

1.13. "Constante du système $S$ ” , „expression qui est censée avoir une signification déterminée quant au contenu dans le système $S^{\prime \prime}$.

Exemples: „Pierre”, „Napoléon I”, „Paris”, „ce livre” etc.

Explication: en définissant la constante, il est nécessaire d'ajouter ,du système $S^{\prime \prime}$, car une expression qui est une constante dans un système donné (p. ex. dans la langue française) pourrait ne pas l'être dans un autre, toutes les expressions humaines ayant un sens arbitraire. La même remarque s'applique aux expressions „variable" (1.14), „nom" (1.33), „opérateur” (1.34), „variable nominale” (1.42) etc. Pour simplifier, nous avons omis cette clause dans la plupart des définitions de ce paragraphe - il faut cependant constamment s'en rendre compte.

1.14. „Variable” — „expression qui n'a pas de sens déterminé quant au contenu mais qui sert exclusivement à indiquer un vide où une constante peut être placée".

1.15. "Isomorphe" - deux expressions sont dites ,isomorphes", quand elles ont la même forme graphique, c. à d. quand dans le langage courant on dit que "c'est la même expression".

Exemple: „la pipe" et "la pipe", „x" et „x".

\subsection{Substitution, catégorie sémantique.}

1.21. "Substituer $b$ à a dans $c$ " ou ,,a par $b$ dans $c$ " signifie: ",former une expression $d$ isomorphe à $c$ en tout, sauf qu'à la place correspondante à a dans $c$, il y a dans $d$ une expression isomorphe à $b$ ".

Exemple: „Substituer 'Paul' à 'Pierre' dans 'Pierre fume la pipe'” signifie: "former la proposition: 'Paul fume la pipe'".

1.22. "Catégorie sémantique du système $S$ " — ,ensemble des expressions qui peuvent être substituées les unes aux autres dans toute expression du système $S$ de sorte que l'expression formée par cette substitution est une expression du système $S^{\prime \prime}$.

Exemple: „Pierre” et „Paul” appartiennent à la même catégorie sémantique de la langue française, car en substituant "Pierre” à „Paul” ou réciproquement dans une expression quelconque de la langue française, on obtient une nouvelle expression de la langue française. Ceci n'est, par contre, pas le cas pour "Pierre" et "dort", car en substituant „dort" à "Pierre" dans "Pierre fume la pipe”, on obtient "dort fume la pipe", donc une expression qui n'est pas une expression de la langue française. 
1.23. "Substitution correcte des variables" - une substitution des variables dans une expression est correcte quand toutes les variables isomorphes de cette expression sont substituées par des expressions (1) isomorphes entre elles et qui (2) appartiennent à la même catégorie sémantique que la variable.

Exemple: la substitution de , $x$ " par „Pierre” dans l'expression,$x=x "$ est correcte, si l'on substitue les deux , $x$ " par deux "Pierre”, en formant l'expression „Pierre = Pierre"; par contre si l'on substituait le premier „ $x$ " seulement, en formant "Pierre $=x^{\prime \prime}$ la substitution ne serait pas correcte.

\subsection{Proposition, nom, opérateur.}

1.31. "Proposition du système $S^{\prime \prime}$ — ,expression qui peut être posée séparément (affirmée) dans le système $S^{\prime \prime}$.

1.32. "Fonction propositionnelle du système $S "$ — ,expression contenant des variables qui devient une proposition du système $S$ quand toutes ses variables sont substituées par des constantes".

Exemples: „Pierre fume la pipe” est une proposition; mais „x fume la pipe” n'est pas une proposition et n'est ni vraie ni fausse; elle devient une proposition quand , $x "$ est substitué par une constante.

1.33. "Nom" - ,expression qui signifie une chose (substance)".

Exemples: „Pierre", „Paris”, „ce crayon".

1.34. „Opérateur" — ,expression qui sert à déterminer une autre expression". 1

Exemples: „beau”, „ccurt”, „aime”, „il n'est pas vrai que”.

1.35. "Argument de l'opérateur F" — ,expression déterminée par l'opérateur $F^{\prime \prime}$.

Exemples: „ciel” est l'argument de „beau” dans „,beau ciel”, „Pierre” de „court” dans „Pierre court”, .,Jean” et „pipe” sont les arguments de „aime” dans: „Pierre aime la pipe"; ..Jean dort" est l'argument de ,il n'est pas vrai que" dans la proposition: "Il n'est pas vrai que Jean dort".

\subsection{Division des variables et des opérateurs.}

1 Il est donc évident que nous employons le mot "opérateur" dans un sens différent de celui qu'il a chez les mathématiciens. Au lieu d',opérateur" on pourrait dire aussi „foncteur" ou, tout simplement, „prédicat” (Quine); par contre l'expression "opération", souvent usitée en français, n'est pas recommandable, car elle peut faire confondre une opération de la raison (acte psychique) avec un symbole écrit d'une réalité extramentale. 
1.41. "Variable propositionnelle” — „variable qui ne peut être substituée que par une proposition ou une fonction propositionnelle".

Exemple: „p" dans: „si $p$, alors Eve fume la pipe” est une variable propositionnelle.

1.42. „Variable nominale" — ,variable qui ne peut être substituée que par un nom individuel".

Exemple:,$\ldots x^{\prime \prime}$ dans $„ x$ fume la pipe" est une variable nominale.

1.43. „Opérateur propositionnel" — ,opérateur dont les arguments ne peuvent être que des propositions ou des fonctions propositionnelles".

Exemple: „si... alors...” est un opérateur propositionnel.

1.44. "Opérateur nominal" — „opérateur dont les arguments ne peuvent être que des noms ou des variables nominales".

Exemples: „boit", „fume", „déteste”.

1.45. "Opérateur n-adique" où $n$ est substitué par un nombre positif entier - opérateur qui détermine $n$ arguments.

Exemples: opérateurs monadiques: „court”, „est méchant”; dyadiques: „aime”, "fume" (,Jean aime la pipe", "Jacques fume la pipe"); triadiques: „donne" (.,Isidore donne la pipe à Boniface"); tétradiques: „est situé entre” (, la Hollande est située entre l'Allemagne, la Belgique et la mer").

\subsection{Définition.}

1.51. "x pour $y "-, x "$ est une abréviation de , $y "$.

1.52. "définition" - ,expression formée par la substitution des variables dans ' $x$ pour $y$ '".

HISTOIRE: L'idée de la catégorie sémantique de Husserl. Les autres idées et l'élaboration systématique de toutes sont dues aux travaux des métalogiciens contemporains (Carnap, Gödel, Leśniewski, Tarski).

LITTÉRATURE: A. Tarski, Der Wahrheitsbegriff in den Formalisierten Sprachen, Studia Philosophica I, 1935. R. Carnap, Introduction to Semantics. Bon résumé dans Quine, Mathematical Logic.

§ 2. RÈGLES D'ÉCRITURE Ayant affaire à des concepts prodigieusement abstraits et complexes, la logique nécessite non seulement l'usage de symboles conventionnels - puisque les mots manquent pour les concepts dont elle traite et ils ne se prêtent guère, même là oủ ils existent, à être maniés avec sûreté - mais encore elle exige d'attacher une 
grande importance aux règles de l'écriture. Ce chapitre donne deux groupes de règles de ce genre: le premier se rapporte à la distinction des deux suppositions, le deuxième à la technique de l'écriture.

\subsection{Suppositions.}

2.11. "L'expression $X$ est prise en supposition formelle" pour: ,l'expression $X$ est considérée comme symbolisant quelque chose qui diffère de $X$ et des expressions isomorphes à $X^{\prime \prime}$.

Exemples: presque tous les mots du langage courant sont pris en supposition formelle; ainsi quand on dit "Pierre dort" on considère le mot "Pierre" en tant que symbolisant l'homme Pierre.

2.12. "L'expression $X$ est prise en supposition matérielle" pour: , $X$ est considéré comme symbole de l'expression $X$ et de tous les isomorphes à $X^{\prime \prime}$.

Exemple: dans „le 'chat' est un substantif", le mot 'chat' est pris en supposition matérielle, car il ne signifie pas l'animal chat, mais le mot 'chat'.

2.13. Règle. Les expressions prises en supposition matérielle doivent être écrites entre guillemets, les expressions prises en supposition formelle sans guillemets.

Exemple: l'expression „le chat boit du lait" est correcte, mais l'expression „le 'chat' boit du lait" est incorrecte, car dans cette dernière le 'chat' étant entre parenthèses, on affirme que le mot 'chat' boit du lait; de même l'expression „le chat est un substantif" est incorrecte, tandis que "le "chat' est un substantif" est correcte.

W. v. O. Quine a proposé d'employer, en dehors des guillemets, des corners de la forme de ' 1 , applicables à des expressions prises en supposition matérielle, mais contenant des variables à substituer, telles que 2.12. Il donne comme raison que, puisque les guillemets changent une expression en un nom d'une expression qui doit être considéré comme un tout (les guillemets inclus), la substitution d'une telle expression n'est pas admissible.

2.14. Règle. Il est désirable que les expressions prises en supposition matérielle soient symbolisées par des expressions non-isomorphes aux expressions elles-mêmes.

2.15. „Métathéorie de T" pour: ,théorie qui traite des expressions de la théorie T"'.

2.16. „Métalogique" pour: „Métathéorie de la logique”.

Exemple: l'ensemble des propositions du $\S 1$ appartient à la métalogique. 


\subsection{Disposition des opérateurs.}

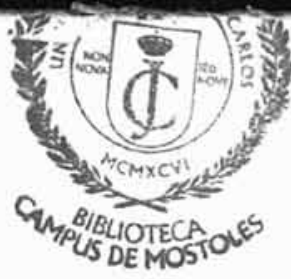

2.21. Règle du système Eukasiewicz: Tous les opérateurs sont placés immédiatement avant (à gauche de) leurs arguments.

Exemples: „aime: Pierre, pipe”, „située entre: Hollande; Belgique, Allemagne, mer".

2.22. Dans le système Łukasiewicz aucune parenthèse n'est nécessaire.

Exemple: l'expression mathématique,$a+a=2 a "$ s'écrit selon $2.21: ~ „=+a a 2 a "$. L'opérateur „.+" étant dyadique, il a comme arguments les deux premiers „a"; on a donc: "+ $+a a=2 a "$; mais l'opérateur $"=$ " étant aussi dyadique, il a comme premier argument "+aa" et comme second „2a"; on obtient ainsi „= +aa $2 a "$.

2.23. Règle du système péano-russellien: Les opérateurs dyadiques sont placés entre leurs arguments; dans les expressions complexes, des parenthèses ou des points sont ajoutés afin d'éviter toute équivoque.

Exemple: dans l'expression „2+2 $2 \times 3$ " il est nécessaire de poser une paire de parenthèses, car sans elle l'expression pourrait avoir deux sens: , $(2+3) \times 2$ " et . $2+(3 \times 2) "$.

\subsection{Parenthèses.}

2.31. "Parenthèse du premier degré" pour „(., et ")".

2.32. "Parenthèse du deuxième degré" pour "[, et "]".

2.33. "Parenthèse du troisième degré" pour , $\{$,. et " $\}$ ".

2.34. "Parenthèse convexe" pour ,., ou "[, ou " $\{$ ".

2.36. "Parenthèse concave" pour .,)., ou "],, ou " $\}$ ".

2.36. Règle: Un opérateur placé avant une parenthèse convexe a comme argument la partie de l'expression qui va de cette parenthèse jusqu'à une parenthèse concave du même degré; un opérateur placé après une parenthèse concave a comme argument la partie de l'expression qui va d'une parenthèse convexe du même degré jusqu'à cette parenthèse.

\subsection{Points.}

2.41. Règle: Une parenthèse du n-ème degré peut être remplacée par un groupe de $n$ points. Deux expressions juxtaposées sont considérées comme séparées par un groupe de 0 points.

2.42. Règle: On ne place des points que près des opérateurs et des quantificateurs $(\S 11.18-19)$, non pas au commencement ni à la fin de l'expression. 
Exemple: l'expression " $(2+3) \times 2$ " s'écrit non pas, $.2+3 . \times 2$ " mais "2+3.X2". Pour des raisons de symétrie on peut, par contre, écrire: "2+3.X.2".

2.43. „Groupe de points de la première classe" pour: ,groupe de points qui tient lieu de l'opérateur de conjonction $(\S 3.6)$ ".

2.44. "Groupe de points de la deuxième classe" pour: ,,groupe de points placé à droite d'un quantificateur" (§11.18-19).

2.45. "Groupe de points de la troisième classe" pour: ,.groupe de points placé à droite ou à gauche d'un opérateur autre que celui de la conjonction".

2.46. Règle: Un opérateur précédé ou suivi d'un groupe de $n$ points de la classe $m$ se rapporte à la partie de l'expression qui va de ce groupe jusqu'au lieu où se trouve (1) un groupe égal de points de la même classe $m$ ou d'une classe supérieure, ou (2) un groupe de plus de $n$ points d'une classe inférieure.

2.47. Règle: Des conventions qui subdivisent les classes de points (2.43-5) peuvent être établies au besoin.

Histoire: La théorie de la supposition est fort ancienne. Le reste du chapitre est une acquisition de la fin du XIXe et du XXe siècle. Les points ont été substitués aux parenthèses par Peano; le système de Eukasiewicz est plus récent encoree, car il date de 1920.

LITTÉRATURE: 2.1, voir le § précédent. 2.21: L'exposé original de Łukasiewicz étant difficilement accessible, on peut consulter: Feys, Logistiek; Bochenski, Nove Lezioni; Syllogism. 2.4: PM p. 9 ss.; toutes les introductions et les traités. Développements: Curry, On the use; Turing, The use. 


\section{II \\ PROPOSITIONS}

\section{§3. OPÉRATEURS DE VÉRITÉ Ce chapitre contient la théorie des liaisons}

entre les propositions non-expliquées par des opérateurs semblables aux mots français: „non”, „ou”, „,si...alors”, „et" etc. Ces opérateurs sont nommés „opérateurs de vérité”, car la vérité d'une proposition formée avec eux dépend exclusivement de la vérité et non pas du sens de leurs arguments.

\subsection{Valeurs.}

3.11. "Valeur" pour: „1" ou " $0 "$.

Explication: On interprète généralement „1" par „vérité" et „, 0 " par „fausseté”; d'où la définition courante de la valeur: „la valeur d'une proposition est sa vérité ou sa fausseté" (Frege).

Contrairement à ce qui arrive dans les interprétations, nous considérons les valeurs comme des symboles (en supposition matérielle, 2.12).

3.12.,$p=x "$ pour: ,la valeur de , $p "$ est , $x "$.

Exemple:,$p=1$ " se lit: „la valeur de 'p' est vérité".

3.13. „F est un opérateur de vérité" pour: „la valeur de toute expression formée de $F$ et des arguments de $F$ dépend exclusivement de la valeur de ces arguments".

Exemple: „exclut" est un opérateur de vérité, car la valeur de la proposition „p exclut $q "$ dépend seulement de la valeur de „., et de ", $q "$; notamment, quand "p" et " $q$ " ont tous les deux la valeur " 1 ", la proposition ..p exclut $q$ " est fausse - dans tous les autres cas elle est vraie, indépendamment du sens de " $p "$ et " $q "$.

\subsection{Négation.}

3.21. , $\{x, y\} "$, où , , $x "$ et , $y "$ sont substitués par des valeurs, pour: ,opérateur monadique tel que $\{x, y\} 1=x$ et $\{x, y\} 0=y "$.

3.22. Il y a quatre opérateurs monadiques: , $\{1,1\} ",,\{1,0\} "$, , $\{0,1\}$ " et , $\{0,0\} " ;$ en général il y a $2^{2 n}$ opérateurs $n$-adiques.

3.23. $" \sim p "$ (ou $\bar{p})$ ou , $N p$ " pour ,,$\{0,1\} p "$. Cet opérateur porte le nom de "négation".

Explication: la négation est un opérateur tel que, placé devant une proposition vraie, il forme une proposition fausse et placé devant une proposition fausse, il forme une proposition vraie. La négation d'une proposition vraie est fausse; la 
négation d'une proposition fausse est vraie. On représente cela par le tableau suivant:

.3.24.

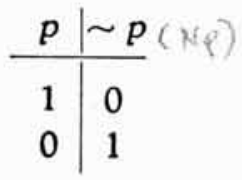

\subsection{Opérateurs dyadiques.}

3.31. , $\{x, y, z, t\} "$ où , , $x ",, y ",,, z ",,, t "$, sont substitués par des valeurs, pour: „opérateur dyadique tel que

$$
\begin{array}{lll}
\text { si } & p=1 \text { et } q=1, & \{x, y, z, t\} p q=x \\
" & p=1 \text { et } q=0, & \{x, y, z, t\} p q=y \\
" & p=0 \text { et } q=1, & \{x, y, z, t\} p q=z \\
" p=0 \text { et } q=0, & \{x, y, z, t\} p q=t^{\prime \prime} .
\end{array}
$$

en tableau:

\begin{tabular}{cc|c}
$p$ & $q$ & $\{x, y, z, t\} p q$ \\
\hline 1 & 1 & $x$ \\
1 & 0 & $y$ \\
0 & 1 & $z$ \\
0 & 0 & $t$
\end{tabular}

\begin{tabular}{|c|c|c|c|c|c|c|c|c|c|}
\hline$p$ & $q$ & $\stackrel{1}{V}$ & $\stackrel{2}{A}$ & $\stackrel{3}{B}^{3}$ & $\stackrel{4}{C}$ & $\stackrel{5}{D}$ & $\stackrel{6}{E}$ & $\stackrel{7}{F}$ & $\stackrel{8}{G}$ \\
\hline 1 & 1 & 1 & 1 & 1 & 1 & 0 & 1 & 0 & 0 \\
\hline 1 & 0 & 1 & 1 & 1 & 0 & 1 & 0 & 0 & 1 \\
\hline 0 & 1 & 1 & 1 & 0 & 1 & 1 & 0 & 1 & 0 \\
\hline 0 & 0 & 1 & 0 & 1 & 1 & 1 & 1 & 1 & 1 \\
\hline 1 & 1 & 0 & 0 & 0 & 0 & 1 & 0 & 1 & 1 \\
\hline 1 & 0 & 0 & 0 & 0 & 1 & 0 & 1 & 1 & 0 \\
\hline 0 & 1 & 0 & 0 & 1 & 0 & 0 & 1 & 0 & 1 \\
\hline 0 & 0 & 0 & 1 & 0 & 0 & 0 & 0 & 0 & 0 \\
\hline$p$ & $q$ & 0 & $X$ & $M$ & $L$ & $K$ & $J$ & $I$ & $H$ \\
\hline & & 16 & 15 & 14 & 13 & 12 & 11 & 10 & 9 \\
\hline
\end{tabular}

ou, en abrégeant:

\begin{tabular}{c|cc}
$\{x, y, z, t\} p q$ & 1 & 0 \\
\hline 1 & $x$ & $y$ \\
0 & $z$ & $t$
\end{tabular}

3.32. Il y a $2^{2^{2}}=16$ opérateurs dyadiques de vérité: 


\subsection{Alternative ou somme logique.}

3.41. "p $p q "$ ou , Apq pour: , $\{1,1,1,0\} p q "$.

Explication: l'alternative correspond au "ou" du langage courant, pris au sens non-exclusif, p. ex. „,'est un prêtre ou un religieux". Une telle proposition est vraie, en effet, quand un de ses arguments est vrai, fausse seulement quand tous les deux sont faux.

3.42 .

\begin{tabular}{|c|c|c|c|}
\hline$p q$ & $p \vee q$ & $v$ & 10 \\
\hline 11 & 1 & 1 & 11 \\
\hline 10 & 1 & 0 & 10 \\
\hline 01 & 1 & & \\
\hline 00 & 0 & & \\
\hline
\end{tabular}

Explication: 3.42 ressemble au tableau de l'addition arithmétique:

$$
\begin{aligned}
& 1+1=2 \\
& 1+0=1 \\
& 0+1=1 \\
& 0+0=0
\end{aligned}
$$

sauf pour la première ligne, où 3.42 donne „l" (car il n'y a pas de valeur supérieure à "1" dans notre système). Pour cette raison on donne le nom de "somme logique" aux expressions du type "p $\vee q$ " resp. ..Apq".

\subsection{Implication matérielle.}

\begin{tabular}{|c|c|c|c|}
\hline$p q$ & $p \supset q$ & כ & 10 \\
\hline 11 & 1 & 1 & 10 \\
\hline 10 & 0 & 0 & $\begin{array}{ll}1 & 1\end{array}$ \\
\hline 01 & 1 & & \\
\hline 00 & 1 & & \\
\hline
\end{tabular}

3.51. ,p $\supset q^{\prime \prime}$ (ou , $p \rightarrow q "$ ) ou (Cpq" pour: ,„\{1,0,1,1\}pq".

Explication: cet opérateur correspond à peu près au „si" français.

3.52 .

\begin{tabular}{|c|c|c|c|}
\hline$p q$ & $|p| q$ & 1 & 10 \\
\hline 11 & 0 & 1 & 01 \\
\hline 10 & 1 & 0 & $\begin{array}{ll}1 & 1\end{array}$ \\
\hline 01 & 1 & & \\
\hline 00 & 1 & & \\
\hline
\end{tabular}

\subsection{Disjonction.}

3.61. ", $p \mid q$ " ou , $D p q "$ pour: , $\{0,1,1,1\} p q "$.

3.62 .

Explication: il n'y a pas de mot correspondant à l'opérateur ,|" resp. „D" en langue française, mais l'idée est souvent exprimée par "ou".

Exemple: „on est un Suisse ou on est un Suédois" c. àd. qu'on ne peut pas 
être les deux à la fois, mais on pourrait être ni l'un ni l'autre, p. ex. si l'on était un Norvégien.

\subsection{Conjonction ou produit logique.}

3.71. „p.q" (ou , ,p \& q") ou , ,Kpq" pour: , $\{1,0,0,0\} p q "$.

\begin{tabular}{cc|c}
$p$ & $q$ & $p \cdot q$ \\
\hline 1 & 1 & 1 \\
1 & 0 & 0 \\
0 & 1 & 0 \\
0 & 0 & 0
\end{tabular}

\begin{tabular}{l|ll}
$\cdot$ & 1 & 0 \\
\hline 1 & 1 & 0 \\
0 & 0 & 0
\end{tabular}

Explication: „.." resp. " $K$ " correspond à ,et". Le tableau 3.72 resemble à celui de la multiplication:

$$
\begin{aligned}
& 1 \times 1=1 \\
& 1 \times 0=0 \\
& 0 \times 1=0 \\
& 0 \times 0=0
\end{aligned}
$$

d'où le nom de "produit logique" donné aux expressions du type „p.q".

3.73. Règle: Le point-opérateur est substitué dans l'écriture avec des points (2.41) par le plus nombreux des groupes de points qui devraient le précéder ou le suivre.

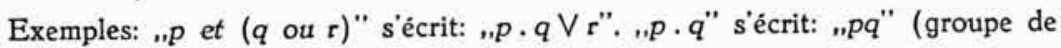
zéro points).

\begin{tabular}{|c|c|c|c|}
\hline$p q$ & $p \equiv q$ & $\equiv$ & 10 \\
\hline $\begin{array}{ll}11 \\
\end{array}$ & 1 & 1 & 10 \\
\hline 10 & 0 & 0 & $\begin{array}{ll}0 & 1\end{array}$ \\
\hline 01 & 0 & & \\
\hline 00 & 1 & & \\
\hline
\end{tabular}

\subsection{Equivalence.}

3.81. ,p $\equiv q$ " (ou , $p \sim q "$ ) ou ,Epq" pour: ,, $\{1,0,0,1\} p q "$.

3.82 .

3.9. Représentation graphique de Gonseth; terminologie.

3.91. En dessinant un carré qui correspond aux tableaux abrégés $3.4-7$

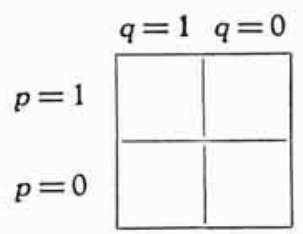


et en remplissant le petit carré qui correspond à " 1 " dans les tableaux cités, on obtient la représentation graphique suivante:

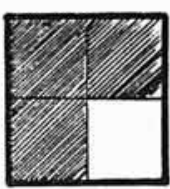

$p \vee q$

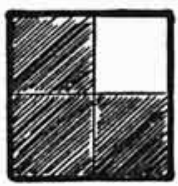

$p \supset q$

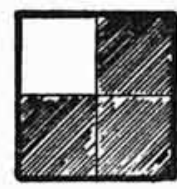

$p / q$

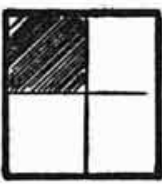

$p \cdot q$

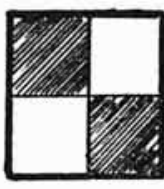

$p \equiv q$

3.92. Terminologie traditionnelle. Si $A p q(p \vee q)$, ,.p" et ", $q$ " sont dites ,subcontraires"; si Cpq ( $p \supset q)$, ,,subalternes"; si Jpq $(p \equiv \sim q)$, ,"contradictoires"; si $D p q(p \mid q)$, ,contraires". On obtient, en particulier, le carré logique suivant:

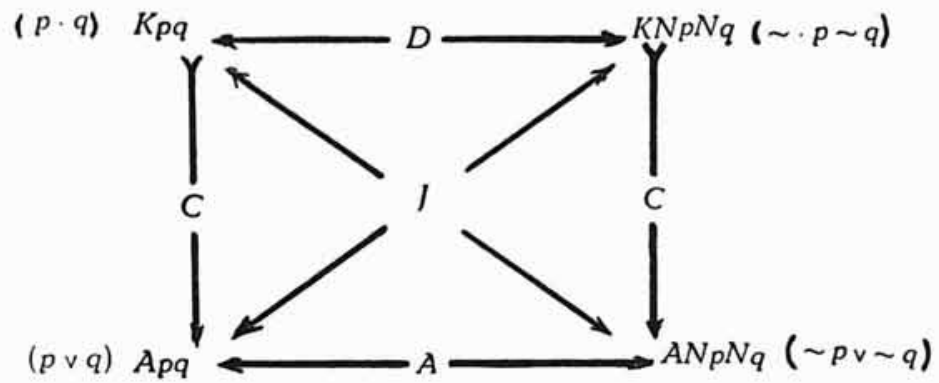

3.93. Tableau comparatif des symbolismes

\begin{tabular}{|c|c|c|c|}
\hline \multirow{2}{*}{ Définition } & \multicolumn{3}{|c|}{ écrit u re } \\
\cline { 2 - 4 } & $\begin{array}{c}\text { péano- } \\
\text { russellienne }\end{array}$ & $\begin{array}{c}\text { de } \\
\text { J. Eukasiewicz }\end{array}$ & $\begin{array}{c}\text { de } \\
\text { D. Hilbert }\end{array}$ \\
\hline 3.23 & $\sim p$ & $N p$ & $\bar{p}$ \\
3.41 & $p \vee q$ & $A p q$ & \\
3.51 & $p \supset q$ & $C p q$ & $p \rightarrow q$ \\
3.61 & $p \mid q$ & $D p q$ & $p \mid q$ \\
3.71 & $p \cdot q$ & $K p q$ & $p \& q$ \\
3.81 & $p \equiv q$ & $E p q$ & $p \sim q$ \\
\hline
\end{tabular}

Exemples de l'écriture:

écriture

péano-russellienne

$p \cdot p \vee r$

$p \vee q \cdot q \supset r$ écriture

de Eukasiewicz

KpApr

KApqCqr traduction verbale:

$p$ et: $p$ ou $r$

$p$ ou $q$, et: si $q, r$ 


$$
\begin{array}{lll}
p \supset q \cdot \supset \cdot \sim q \supset \sim p & C C p q C N q N p & \text { si }(\text { si } p, \text { alors } q) \text {, alors: } \\
& & (\text { si non- } q \text {, alors non- } p) \\
p \supset q: \supset: q \supset r \cdot \supset \cdot p \supset r & C C p q C C q r C p r & \text { si: }(\text { si } p \text {, alors } q) \text { alors: } \\
& \text { si }(\text { si } q, \text { alors } r) \text { alors: } \\
& (\text { si } p, \text { alors } r) .
\end{array}
$$

Ce dernier exemple montre la nécessité d'employer un symbolisme artificiel.

HISTOIRE: La théorie fut déjà connue des Stoïciens, qui donnèrent, entre autres, 3.52; au XIXe siècle elle fut réinventée et élaborée par Peirce, suivi par Wittgenstein, Post et Łukasiewicz au XXme. La méthode exposée ici est due à Feys, la représentation graphique à Gonseth.

LITTÉRATURE: La majorité des introductions et des traités, en particulier:

Feys, Logistiek; Wittgenstein, Tractatus; Lukasiewicz-Tarski, Untersuchungen Łukasiewicz, Die Logik. - § 3.8: Gonseth, Qu'est-ce que la logique? - Pour l'histoire: Łukasiewicz, Zur Geschichte; Bochenski, Consequentiae.

\section{§ 4. Evaluation Le problème discuté dans le présent chapitre est de savoir} comment s'assurer qu'une expression est une loi logique, c. àd. que quelles que soient les substitutions correctes de ses variables, elle devient une proposition vraie. Le problème reçoit plusieurs solutions. Est exposée ici la plus perfectionnée et la plus commode, la méthode de l'évaluation par substitution.

\subsection{Définitions.}

4.11. „Loi logique” pour: „,fonction propositionnelle qui devient une proposition vraie quand ses variables ont été toutes substituées d'une manière correcte (1.23) quelconque par des constantes".

4.12. „Evaluet" pour: "montrer qu'une expression est une loi ou ne l'est pas".

4.13. „Expression élémentaire” pour: ,.expression composée de

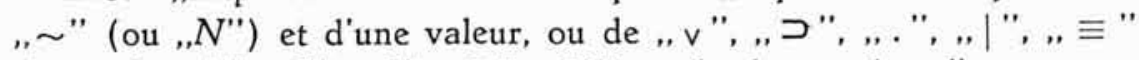
(resp. de ",A", ,C", ,,K", ,D", ,E") et de deux valeurs".

\subsection{Technique de l'évaluation.}

4.21. Règle de l'évaluation: (a) déterminer toutes les combinaisons possibles des valeurs, (b) substituer les valeurs de la première combinaison aux variables de l'expression évaluée, (c) substituer aux expressions élémentaires ainsi formées leurs valeurs d'après les définitions du $\S 3$, (d) répéter cette opération jusqu'au moment où un seul chiffre reste, (e) si ce chiffre est ' $O$ ' l'expression n'est pas une loi, (f) s'il est ' 1 ' substituer les autres combinaisons de la même manière, 
(g) si toutes les combinaisons donnent comme résultats ' 1 ' l'expression est une loi.

4.22. Les combinaisons des valeurs sont $2^{n}$ pour $n$ variables nonisomorphes. Elles sont les suivantes:

pour toutes les variables isomorphes: (1) $p=1$, (2) $p=0$

pour deux variables non-isomorphes: (1) $p=1, q=1$

(2) $p=1, q=0$

(3) $p=0, q=1$

(4) $p=0, q=0$

pour trois variables non-isomorphes elles sont 8: (1) $p=1, q=1$, $r=1 ;$ (2) $p=1, q=1, r=0$ etc.

4.23. Valeurs des expressions élémentaires. Le tableau suivant facilite la substitution des valeurs aux expressions élémentaires:

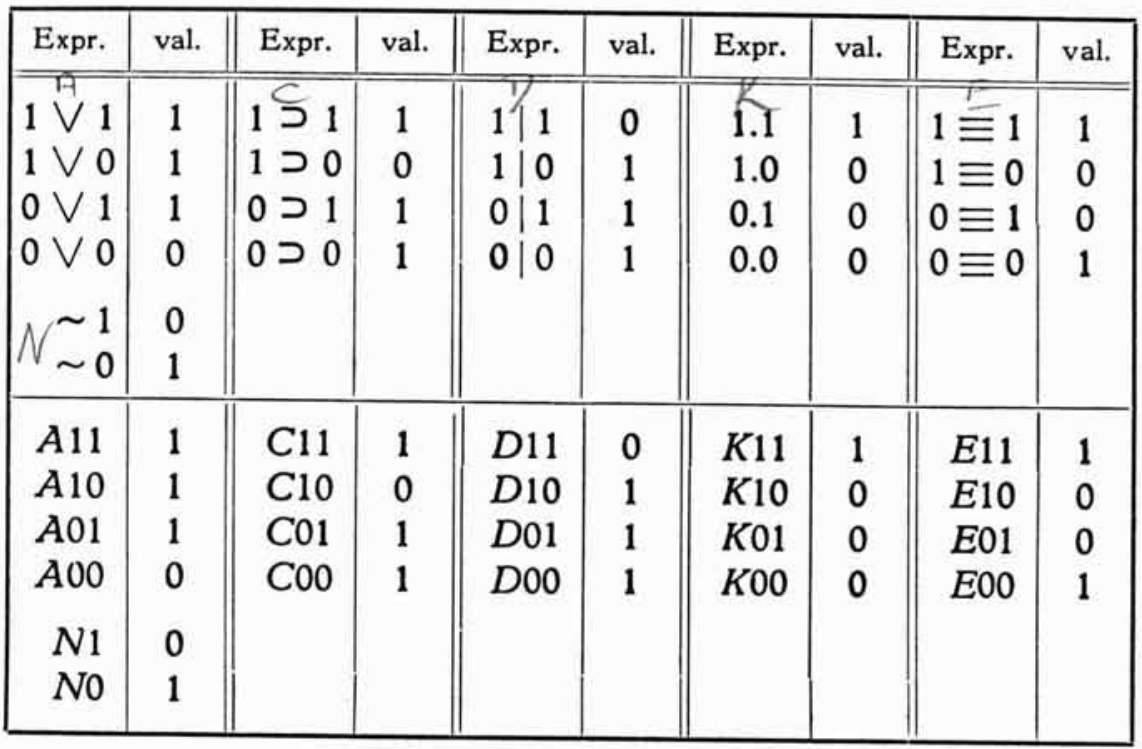

Exemple: soit à évaluer l'expression "CCpqCNqNp". Il y a deux variables non-isomorphes "p" et " $q$ "; donc, 4 substitutions:
(a) $p=1, q=1$
(b) $p=1, q=0$
(c) $p=0, q=1$
(d) $p=0, q=0$

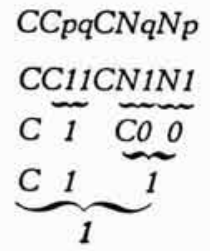

$C \mathrm{Cp} q \mathrm{CN} q \mathrm{~Np}$

$\mathrm{CC}_{p q C N} \mathrm{CN} p$

$\mathrm{CC}_{p q C N} \mathrm{CN} \mathrm{N}$

CC11CN1N1

CC1OCNON1

CCOICNINO

C $\widetilde{1}$ CO 1

C 0 C10

$\underbrace{C 0}_{1} \quad$

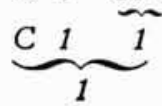

CCOOCNONO

C 1 C1 1

$\underbrace{C 1}_{1}$ 
La méthode est la même quand on emploie l'écriture péano-russellienne - mais alors il est nécessaire d'omettre les points et les parenthèses au fur et à mesure que les substitutions sont faites; pour cette raison il est plus commode d'opérer l'évaluation sur des expressions de l'écriture Lukasiewicz.

HISTOIRE: cf. $\S 3$.

LITTÉRATURE: Les exposés les plus lucides sont: Hilbert-Ackermann, Grundzüge; Quine, Mathematical Logic, et Feys, Logistiek.

\section{§ 5. EQUIVALENCES Ce chapitre contient l'énumération des lois logiques en \\ forme d'équivalences les plus simples et les plus utiles.}

Elles sont données en double écriture, d'abord en péano-russellienne, ensuite dans celle de Łukasiewicz. 5.1 contient les lois qui ne comprennent pas de variables non-isomorphes, $5.2-6$, les lois à deux et à trois variables non-isomorphes, groupées d'après l'opérateur du premier argument.

\subsection{Lois avec toutes les variables isomorphes.}

5.11. $p \equiv p$

5.12. $\sim \sim p \equiv p$

5.13. $\sim \sim \sim p \equiv \sim p$

5.14. $\sim p \equiv p \mid p$

5.15. $p \vee p \cdot \equiv \cdot p$

5.16. $p p \equiv p$
Epp

ENNPp

$E N N N p N p$

$E N p D p p$

EAppp

EKppp
Principe d'identité.

Principe de la double négation.

Principe de la triple négation.

1ère loi de la tautologie.

2ème loi de la tautologie.

\subsection{Lois de la somme (Alternative).}

5.211. $p \vee q \cdot \equiv \cdot \sim p \supset q$

5.212. $p \vee q \cdot \equiv \cdot \sim p \mid \sim q$

5.213. $p \vee q \cdot \equiv \sim \sim \sim p \sim q$

5.214. $p \vee q: \equiv: p \supset q \cdot \supset q$

5.22. $p \vee q \cdot \equiv \cdot q \vee p$

5.23. $p \cdot \vee \cdot q \vee r: \equiv: p \vee q \cdot \vee \cdot$

5.24. $p \cdot \vee \cdot q r: \equiv: p \vee q \cdot p \vee \tau$

5.25. $p \cdot \vee \cdot p \vee q: \equiv: p \vee q$

5.26. $p \cdot \vee \cdot p q: \equiv: p$

5.27. $\sim p \vee q \cdot \equiv \cdot \sim p \cdot \sim q$
$E A p q C N p q$

$E A p q D N p N q$

EApqNKNpNq 3ème loi de Morgan.

EApqCCpqq

EApqAqp

EApAqrAApqr

$E A p K q r K A p q A p r$

$E A p A p q A p q$

$E A p K p q p$

ENApqKNpNq
Loi de la permutation de la somme.

Loi associative de la somme.

Loi distributive de la somme.

lère loi de la simplification de la somme. 2ème loi de la simplification de la somme. lère loi de Morgan. 


\subsection{Lois de l'implication.}

5.311. $p \supset q \cdot \equiv \cdot \sim p \vee q$

5.312. $p \supset q \cdot \equiv \cdot p \mid \sim q$

5.313. $p \supset q \cdot \equiv \cdot \sim p \sim q$

5.314. $p \supset q \cdot \equiv \cdot p \equiv p q$

5.315. $p \supset q: \equiv: q \cdot \equiv \cdot p \vee q$

5.32. $p \supset q \cdot \equiv \sim q \supset \sim p$

5.321. $p \supset \sim q \cdot \equiv \cdot q \supset \sim p$

5.322. $\sim p \supset q \cdot \equiv \cdot \sim q \supset p$

5.33. $p \cdot \supset \cdot q \supset r: \equiv: q \cdot \supset \cdot p \supset r$

5.34. $p q \supset r: \equiv: p \cdot \supset \cdot q \supset r$

5.35. $p q \supset r: \equiv: q \cdot \supset \cdot p \supset r$

5.36. $p \cdot \supset \cdot p \supset q: \equiv: p \supset q$

5.37. $p q \supset r . \equiv \cdot \sim r q \supset \sim p$

5.38. $p q \supset r . \equiv \cdot p \sim r \supset \sim q$

5.39. $\sim \cdot p \supset q \cdot \equiv \cdot p \sim q$
$E C p q A N p q$

$E C p q D p N q$

$E C p q N K p N q$

ECpqEpKpq

$E C p q E q A p q$

ECpqCNqNp

${ } C p N q C q N p$

$E C N p q C N q p$

$\mathrm{EC}_{p} \mathrm{Cqr} \mathrm{CqCpr}$

ECKpqrCpCqr

ECKpqrCqCpr

$E C p C p q C p q$

ECKpqrCKN $r q N p$

$\mathrm{ECKpqrCKpNrNq}$ ENCpqKpNq
Loi de la contraposition simple.

2ème loi de la contraposition simple.

3ème loi de la contraposition simple.

Loi de la commutation simple.

1 ère loi del'exportation. 2ème loi de l'exportation. 1ère loi de la contraposition syllogistique. 2ème loi de la contraposition syllogistique.

\subsection{Lois de la disjonction.}

5.41. $p \mid q \cdot \equiv \cdot \sim p \vee \sim q$

5.42. $p \mid q \cdot \equiv \cdot p \supset \sim q$

5.43. $p \mid q \cdot \equiv \cdot \sim p q$

5.44. $p|q \cdot \equiv \cdot q| p$

5.45. $p \mid p \cdot \equiv \cdot \sim p$

5.46. $\sim \cdot p \mid q \cdot \equiv \cdot p q$

\subsection{Lois du produit.}

5.511. $p q \cdot \equiv \cdot \sim \cdot p \vee \sim q$

5.512. $p q \cdot \equiv \cdot \sim p \supset \sim q$

5.513. $p q \cdot \equiv \cdot \sim \cdot p \mid q$

5.52. $p q \equiv q p$

5.53. $p \cdot q r \cdot \equiv \cdot p q \cdot \tau$
$E D p q A N p N q$

$E D p q C p N q$

EDpqNKpq

$E D p q D q p$

$E D p p N p$

$E N D p q K p q$
EKpqNANpNq 4ème loi de Morgan. $E K p q N C p N q$

$E K p q N D p q$

$E K p q K q p$

$E K p K q r K K p q r$
Loi de la permutation du produit.

Loi associative du produit. 
5.54. $p \cdot q \vee r . \equiv \cdot p q \vee p r$

5.55. $p \cdot p \vee q \cdot \equiv \cdot p$

5.56. $p \cdot p q \cdot \equiv \cdot p q$

5.57. $\sim \cdot p q \cdot \equiv \cdot \sim p \vee \sim q$

5.58. $\sim \cdot p q \cdot \equiv \cdot p \mid q$
$E K p A q r A K p q K p \tau \quad$ Loi distributive du

$E K p A p q p$

$E K p K p q K p q$

$E N K p q A N p N q$

ENKpqDpq

1ère loi de la simplification du produit. 2ème loi de la simplification du produit. 2ème loi de Morgan.

produit.

5.6. Lois de l'équivalence.

5.611. $p \equiv q \cdot \equiv \cdot p q \vee \sim p \sim q$

5.612. $p \equiv q \cdot \equiv \cdot p \supset q \cdot q \supset p$

$E E p q A K p q K N p N q$

5.62. $p \equiv q \cdot \equiv \cdot q \equiv p$ EEpqKCpqCqp

EEpqEqp

Loidela permutation de l'équivalence.

5.63. $p \cdot \equiv \cdot q \equiv r: \equiv: p \equiv q \cdot \equiv \cdot r \quad E E_{p} E q r E E p q r$

5.64. $p \equiv q \cdot \equiv \cdot \sim p \equiv \sim q$ $E E p q E N p N q$

5.65. $p \equiv q \cdot \equiv \cdot \sim q \equiv \sim p \quad E E p q E N q N p$

5.66. $\sim p \equiv q \cdot \equiv \cdot \sim q \equiv p \quad E E N p q E N q p$

5.67. $p \equiv \sim q \cdot \equiv \cdot q \equiv \sim p \quad E E p N q E q N p$

Loi associative de

l'équivalence.

\subsection{Règles de transformation.}

5.71. „Opérateur principal de $X$ " pour (a) ,premier ,„”" de $X "$ si $X$ est une des lois $5.1-5.5$ (b) ,troisième ," $\equiv "$ de $X$ " si $X$ est 5.63 (c) ,deuxième "三" de $X^{\prime \prime}$ si $X$ est une des lois 5.6, excepté 5.63.

5.72. Règle de l'inversion: $\mathrm{Si} X$ est une des lois du $\S 5$, colonne gauche, l'expression formée par la substitution de la partie de $X$ qui suit l'opérateur principal de $X$ à celle qui le précède et réciproquement, est une loi.

Exemple: puisque (5.16) ,pp $\equiv p "$ est une loi, ,p $\equiv p p "$ est aussi une loi.

5.73. Règle de la substitution de l'implication: $\mathrm{Si} X$ est une loi du $\S 5$., colonne gauche, l'expression formée par substitution de son opérateur principal par, , ” est une loi; si $X$ est une loi du $\S 5$, colonne droite, l'expression formée par la substitution dé son premier „E" par "C" est une loi.

LITTÉRATURE: Une énumération presque complète des lois utilisées en pratique dans les PM ${ }^{\star} 2-{ }^{\star} 5$ et dans les traités, en particulier chez Feys. 
§6. „PREMIERS PRINCIPES” ET IMPLICATIONS

Les lois énumérées ici sont les plus importantes à côté des équivalences. 6.1. donne trois lois connues (avec 5.11.) comme „premiers principes" de la logique traditionnelle, dans leur forme propositionnelle. L'arrangement est le même qu'au $§ 5$.

\section{1. „Premiers principes".}

$6.11 \sim \cdot p \sim p \quad N K p N p \quad$ Principe de non-contradiction.

6.12. $p \mid \sim p \quad D p N p$

6.13. $p \vee \sim p \quad A p N p \quad$ Principe du tiers exclu.

6.2. Lois caractéristiques de l'implication.

6.21. $p \cdot \supset \cdot q \supset p \quad C p C q p \quad$ 1ère loi paradoxale (,verum sequitur ad quodlibet").

6.22. $\sim p \cdot \supset \cdot p \supset q \quad C N p C p q$ 2ème loi paradoxale (,,ex falso sequitur quodlibet").

6.23. $p \supset \sim p . \supset . \sim p C C p N p N p$ 1ère loi de la réduction à

6.24. $p \cdot \supset . \sim p \supset q \quad C p C N p q$

l'absurde.

6.25. $p \sim p \supset \sim p \quad C K p N p N p$ 2ème loi de la réduction à l'absurde.

6.26. $p \cdot \supset \cdot p \vee q \quad C p A p q \quad$ Loi du nouveau facteur.

6.27. $p q \supset p \quad C K p q p \quad$ lère loi de l'a fortiori.

6.271. $p q \supset q \quad C K p q q \quad$ 2ème loi de l'a fortiori.

6.281. $p q \cdot \supset \cdot p \supset q \quad C K p q C p q$

6.282. $p \equiv q \cdot \supset \cdot p \supset q \quad C E p q C p q$

\subsection{Lois du syllogisme.}

6.31. $q \supset r: \supset: p \supset q \cdot \supset \cdot p \supset r$

$\mathrm{CC} q \mathrm{rCC} p q \mathrm{Cpr}$

6.32. $p \supset q: \supset: q \supset r \cdot \supset \cdot p \supset r$

$\mathrm{CC}_{p q C C q r C p r}$

6.33. $p \supset q: \supset: q \supset r: \supset: r \supset s \cdot \supset \cdot p \supset s \quad$ CCpqCCqrCCrsCps

6.34. $p \supset q:: \supset:: q \supset r: \supset: \cdot r \supset s: \supset: s \supset t \cdot \supset \cdot p \supset t$ CCpqCCqrCCrsCCstCpt

6.35. $p \supset q \cdot q \supset \tau \cdot \supset \cdot p \supset \tau$

$C K C p q C q r C p r$

6.36. $p \supset q \cdot q \supset r \cdot r \supset s \cdot \supset \cdot p \supset s$

$\mathrm{CKKCpqCqrCrsCps}$

6.37. $p \supset q \cdot q \supset r \cdot r \supset s \cdot s \supset t \cdot \supset \cdot p \supset t$

CKKKCpqCqrCrsCstCpt

6.38. $p \supset q: \supset: \tau \vee p \cdot \supset \cdot r \vee q$

CCpqCArpArq

\subsection{Modes du syllogisme conditionnel.}

6.41. $p: \supset: p \supset q \cdot \supset \cdot q$

$\mathrm{CpCC}_{p} q q$

Modus ponendo

ponens $1^{\circ}$. 
6.42. $p \supset q \cdot p \cdot \supset \cdot q$

CKCpqpq

Modus ponendo

ponens $2^{\circ}$.

6.421. $p \supset \sim q \cdot p \cdot \supset \cdot \sim q$

$C K C p N q p N q$

6.422. $\sim p \supset q \cdot \sim p \cdot \supset \cdot q$

CKCNpqNpq

6.423. $\sim p \supset \sim q \cdot \sim p \cdot \supset . \sim q$

$C K C N p N q N p N q$

6.43. $\sim q: \supset: p \supset q \cdot \supset \cdot \sim p$

$C N q C C p q N p$

Modus tollendo tollens

$1^{\circ}$.

6.44. $p \supset q \cdot \sim q \cdot \supset \cdot \sim p$

$C N q C C p q N p \quad$ Modus tollendo tollens $2^{\circ}$.
6.441. $p \supset \sim q \cdot q \cdot \supset \cdot \sim p$
$C K C p N q q N p$
6.442. $\sim p \supset q \cdot \sim q \cdot \supset \cdot p$
CKCNpqNqp
6.443. $\sim p \supset \sim q \cdot q \cdot \supset \cdot p$
CKCNpNqqp

6.5. Modes du syllogisme disjonctif et copulatif.

6.51. $\sim p: \supset: p \vee q \cdot \supset \cdot q$

6.511. $\sim q: \supset: p \vee q \cdot \supset \cdot p$

6.52. $p \vee q \cdot \sim p \cdot \supset \cdot q$

6.521. $p \vee q \cdot \sim q \cdot \supset \cdot p$

6.523. $\sim p \vee q \cdot p \cdot \supset \cdot q$

6.524. $\sim p \vee \sim q \cdot p \cdot \supset \cdot \sim q$

6.53. $p: \supset: p \mid q \cdot \supset \cdot \sim q$

6.531. $q: \supset: p \mid q \cdot \supset \cdot \sim p$

6.532. $p: \supset: \sim \cdot p q \cdot \supset \cdot \sim q$

6.54. $p \mid q \cdot p \cdot \supset \cdot \sim q$

6.541. $p \mid \sim q \cdot p \cdot \supset \cdot q$

6.542. $\sim p \mid q \cdot \sim p \cdot \supset . \sim q$

6.543. $\sim p \mid \sim q \cdot \sim p \cdot \supset \cdot q$
6.522. $p \vee \sim q \cdot \sim p . \supset . \sim q$
$C N p C A p q q$

CNqCApqp

CKApqNpq

CKApqNqp

CKApNqNpNq

CKANpqpq

CKANpNqpNq

$\mathrm{CpCDpqNq}$

$C q C D p q N p$

CpCNKpqNq

CKDpqpNq

CKDpNqpq

$C K D N p q N p N q$

$C K D N p N q N p q$
Modus tollendo ponens $1^{\circ}$.

Modus tollendo ponens $2^{\circ}$.

Modus ponendo tollens $1^{\circ}$.

\subsection{Lois de la Composition et Dilemmes.}

6.61. $p \supset q \cdot p \supset r \cdot \supset \cdot p \supset q r \quad C K C p q C p r C p K q r$

Loi de la multiplication du conséquent.

6.62. $p \supset q \cdot r \supset s: \supset: p r \supset q s \quad C K C p q C r s C K p r K q s \quad$ Loi de la multiplication des deux côtés. 
6.63. $p \supset q \cdot r \supset s: \supset: p \vee r \cdot \supset . q \vee s \quad C K C p q C r s C A p r A p s \quad$ Loi de l'addition des deux côtés.

6.64. $p \supset r \cdot q \supset r: \supset: p \vee q \cdot \supset$. $r \quad C K C p r C q r C A p q r$ ler dilemme constructif.

6.65. $p \supset r \cdot \sim p \supset r \cdot \supset \cdot r \quad C K C p r C N p r t$

2ème dilemme constructif.

6.67. $\sim q \sim r: \supset: \cdot p \cdot \supset \cdot q \vee r: \supset: \sim p \quad C K N q N r C C p A q r N p$

6.671. $p . \supset \cdot q r: \sim q \sim \tau: \supset: \sim p \quad C K C p K q r K N q N r N p$

6.68. $p \supset q \cdot p \supset \sim q \cdot \supset . \sim p \quad C K C p q C p N q N p$ destructif.

3ème loi de réduction à l'absurde.

LITTÉRATURE: cf. $§ 5$.

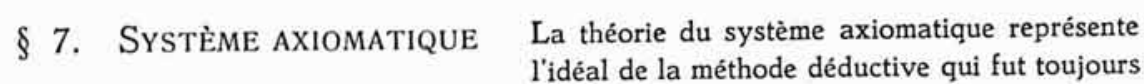
enseignée par la logique. Cette méthode a été d'abord appliquée dans sa pleine rigueur à la logique elle-même; actuellement elle est déjà tellement perfectionnée qu'on peut l'appliquer dans des domaines nombreux. Le chapitre présente la théorie d'une manière succinte, en sacrifiant la rigueur à la clarté; un développement parfaitement rigoureux demanderait de très longs exposés.

\subsection{Définitions.}

7.11. „Système axiomatique” pour: ,,ensemble d'expressions divisé en deux classes de telle manière que les éléments de la deuxième sont formés par l'application des règles explicitement formulées aux éléments de la première".

7.12. Le système axiomatique comprend des termes, des expressions et des lois, des règles de la définition des termes, de la formation des expressions et de la déduction des lois.

\subsection{Termes et définitions.}

7.21. „Terme du système $S$ " pour: ,expression du système $S$ dont ancune partie n'est une expression du système $S^{\prime \prime}$.

7.22. "Définir $X$ par $Y$ " pour: ,former une expression qui indique que $Y$ peut être substitué par $X^{\prime \prime}$.

7.23. "Terme primitif du système $S$ " pour: ,terme du système $S$ qui n'est pas défini dans le système $S^{\prime \prime}$ (cf. 1.31).

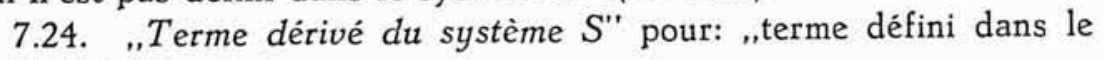
système $S^{\prime \prime}$.

7.25. "Règle de la définition du système $S$ " pour: „règle qui indique le mode correct de définir les termes dérivés du système $S^{\prime \prime}$. 
7.26. Règle: Tous les termes primitifs et les règles de définition du système axiomatique doivent être explicitement donnés, tous les termes qui ne sont pas des termes primitifs doivent être explicitement définis.

\subsection{Expressions et règles de formation.}

7.31. „Règle de formation du système $S$ " pour: „,règle qui indique comment les termes du système $S$ peuvent être groupés en expressions du système $S^{\prime \prime}$.

Dans beaucoup de systèmes toutes les expressions sont des propositions.

Exemple: une des règles du système Łukasiewicz est „un groupe de termes composé de ,C' et de deux variables est une proposition".

7.32. Règle générale de formation: Toutes les expressions du système $S$ doivent être exclusivement formées de termes de $S$ et d'une manière indiquée dans les règles de formation de $S$.

\subsection{Lois et déduction.}

7.41. „Loi du système $S "$ pour: „proposition affirmée dans le système $S^{\prime \prime}$.

7.42. "Déduire $Y$ de $X$ dans le système $S$ " pour: „montrer que si $X$ est une loi de $S$, les règles de $S$ permettent d'affirmer $Y$ ".

7.43. „Axiome du système $S$ " pour: „loi du système $S$ qui n'est pas déduite dans le système $S^{\prime \prime}$.

7.44. "Thèse du système $S$ " pour: „loi du système $S$ déduite des axiomes de $S$ par l'application des règles de $S^{\prime \prime}$.

7.45. "Règle de la déduction du système $S$ " pour: ,,règle qui indique le mode correct de déduire dans le système $S^{\prime \prime}$.

7.46. Règle: Tous les axiomes et toutes les règles de la déduction du système axiomatique doivent être explicitement données; toutes les autres propositions affirmées doivent être explicitement déduites.

7.47. Règle: L'application des lois et des définitions dans la déduction d'une thèse doit être explicitement formulée dans une expression spéciale dite „,verset probatif".

Exemples: le $\S 8$ contient plusieurs exemples de versets probatifs avec explications.

7.48. "X implique inférentiellement $Y$ dans le système $S$ " pour: ,les règles de la déduction du système $S$ permettent de déduire $Y$ de $X^{\prime \prime}$. 
Explication: il ne faut pas confondre l'implication matérielle avec l'implication inférentielle. La première se vérifie, par exemple, entre toutes les propositions vraies, ce qui n'est pas le cas pour la deuxième. Le „si" du langage courant se rapproche plus de l'implication inférentielle que de l'implication matérielle.

\subsection{Formalisme.}

7.51. „Système formalisé" pour: „système axiomatique dont les règles concernent exclusivement la forme graphique des expressions et dont tous les axiomes et les règles sont explicitement formulées".

7.52. Règle: Le système formalisé doit être développé, une fois ses axiomes et règles établies, sans aucune référence au sens des expressions utilisées, en vertu des règles seules.

7.53. Le système axiomatique formalisé est privé comme tel de tout sens et peut recevoir diverses interprétations.

Exemple: le système exposé dans les $\S \S 4-6$ doit être considéré comme un ensemble de lettres qui ne symbolisent rien; il ne faut, par exemple, pas prendre "C" pour le symbole de l'implication au sens du ,si" courant, mais exclusivement pour un opérateur tel que le défini du tableau 3.52. Aux $\S \S 9,14$ et 16 nous donnerons trois interprétations différentes de celle qui a été admise, pour faciliter la compréhension, au $\S 3$.

\subsection{Consistance.}

7.61. „Système non-contradictoire" pour: „système axiomatique dont les règles de la déduction ne permettent pas de déduire de ses axiomes une proposition avec la négation de cette proposition".

7.62. Chaque proposition peut être prouvée dans chaque système complet qui est contradictoire.

Explication: en vertu de 6.24 une proposition affirmée en même temps que sa négation permet de déduire " $q$ "; en substituant, on obtient chaque proposition voulue. Par là, la distinction entre les propositions vraies et fausses s'évanouit et la science n'est plus possible. C'est la raison qui a fait dire à Aristote que le principe de non-contradiction (6.11) est le premier principe de la logique.

\subsection{Système complet et indépendant.}

7.71. „Système complet au sens large" pour: „,système axiomatique qui comprend toutes les propositions vraies du domaine donné".

7.72. "Système complet au sens strict" pour: „système axiomatique tel que toute proposition qui n'est pas une de ses lois est la négation d'une de ses lois".

7.73. „Système à axiomes indépendants" pour: ,,système axiomatique dont aucun axiome ne peut être déduit, en vertu des règles du système, des autres axiomes du système". 


\subsection{Règles.}

7.81. Règle: Tout système axiomatique doit être formalisé et noncontradictoire.

7.82. Règle: Il faut tâcher d'établir des systèmes complets au sens strict, et aux axiomes indépendants.

HISTOIRE: Le système axiomatique, cultivé surtout par les mathématiciens (système d'Euclide), reçut son élaboration formelle et rigoureuse des métalogiciens contemporains (cf. § 25 ).

LitTérature: Exposés élémentaires: Carnap, Abriss; Tarski, Introduction; Dieudonné, Les méthodes, - Elaborations rigoureuses métalogiques cf. § 24; cf. aussi: Bernays, A System; Schröter, Ein allg. Kalkülbegriff; Axiomatisierung; Woodger, The Technique; Carnap, Formalization. Une ample littérature existe sur les méthodes pour prouver la consistance, le caractère complet et l'indépendence des axiomes; l'oeuvre la plus notable dans ce domaine est Gödel, Ueber
formal...

\section{§ 8. Système de la logique DES PROPOSITIONS}

parmi les connues. Seuls les fondeméthode employée est la plus rigoureuse quelques démonstrations initiales sont données.

\subsection{Termes primitifs, règles de définition et de formation.}

8.11. Termes primitifs: "D" - opérateur binaire; , $p ",,, q ", \ldots,{ }^{\prime \prime}$, "s" - variables propositionnelles.

8.12. Règle de la définition: On peut introduire dans le système un nouveau terme en formulant un groupe de termes, dit ",définition" et composé successivement: (1) d'une expression qui comprend le terme nouveau, tous les autres étant déjà des termes du système, (2) de ,=", (3) d'une expression composée exclusivement de termes primitifs ou déjà définis.

8.13. Règles de la formation: (1) une variable est une proposition, (2) un groupe de termes composé de , $N^{\prime \prime}$ et d'une proposition est une proposition, (3) un groupe de termes composé de „, $A$ ", , $C$ ", , ,D", , $E$ " ou , $K$ " et de deux propositions est une proposition.

\subsection{Définitions:}

8.21. $N p=D_{p p}$ (cf. 5.14)

8.22. $A p q=D N p N q$ (cf. 5.213)

8.23. $C p q=A N p q$ (cf. 5.311)

8.24. $K p q=N A N p N q$ (cf. 5.511)

8.25. $E p q=K C p q C q p$ (cf. 5.612) 


\subsection{Règles de la déduction.}

8.31. Règle de la substitution: Une variable peut être substituée par une proposition.

8.32. Règle de la substitution par définition: Une expression peut être substituée dans une proposition par l'expression qui la définit et réciproquement, sans substituer les autres expressions isomorphes de la même proposition.

8.33. Règle de la dérivation: $\mathrm{Si}$ une proposition composée de „C" et de deux propositions est une loi du système et si une proposition isomorphe à la première de ces propositions est une loi du système, une proposition isomorphe à la deuxième peut être posée comme loi du système.

\subsection{Axiomes:}

8.41. CAppp (cf. 5.15)

8.42. $C p A p q$ (cf. 6.26)

8.43. CApqAqp (cf. 5.22)

8.44. CCpqCArpArq (cf. 6.38)

\subsection{Déduction:}

$$
8.44 r / N r \times 8.23 p / r, q / p \times 8.23 p / r=8.51
$$

\subsection{1. $\mathrm{CC} p q \mathrm{CCrpCrq}$ (cf. 6.38)}

Explication: le verset probatif de la thèse 8.51 se lit: „prendre l'axiome 8.44; substituer , $r$ " par " $N r^{\prime \prime}$; appliquer la définition 8.23 , dans laquelle on a opéré la substitution de , $p$ " par $" q "$ et de,$q "$ par,${ }^{\prime \prime}{ }^{\prime \prime}$; appliquer la même définition 8.23 avcc la substitution de ".p" par " $r "$ en deuxième lieu; on obtiendra ainsi la thèse à démontrer $8.51 . "$

\section{$8.51 p / A p p, q / p, r / p=C 8.41-C 8.42 q / p-8.52$ 8.52. $C_{p p}$}

Explication: après avoir accompli les substitutions indiquées au commencement, on obtient l'expression:

\section{$C C A p p p C C p A p p C p p$}

qui est composée de (1) „C", (2) de „CAppp” c. à d. d'une expression isomorphe à 8.41 , (3) de "C", (4) de "C $C A p p$ " qui est isomorphe à 8.42 après avoir substitué dans cette "q" par "p", (5) de la thèse "Cpp" qui est déduite ici en vertu d'une double application de la règle de dérivation (8.33).

8.53. $A N_{p p}$

$$
8.52 \times 8.23 q / p=8.53
$$

$$
8.43 p / N p, q / p=C 8.53-8.54
$$


8.54. $A p N p$
$8.54 p / N p \times 8.23 q / N N p=8.55$

8.55. $C p N N p$ $8.44 p / N p, q \mid N N N p, \tau / p=C 8.55 p / N p,-C 8.54-8.56$

8.56. ApNNNp

$$
8.43 q / N N N p \times 8.23 p / N N p, q / p=C 8.56-8.57
$$

8.57. CNNpp
$8.44 q / N N p, r / N q=C 8.55-8.58$

8.58. CANqpANqNNp

$8.51 p / A N q N N p, q \mid A N N p N q, \tau / A N q p=C 8.43 p / N q$.

$$
q / N N p-C 8.58-8.59
$$

8.59. CANqpANNpNq

$8.59 p / q, q / p \times 8.23 \times 8.23 p / N q, q / N p=8.60$

8.60. $C C_{p q C N q N p}$

$8.41 p / N p \times 8.23 q / N p=8.61$

8.61. $C C p N p N p$

$8.51 p / A p q, q \mid A q p, \tau / p=C 8.43-C 8.42-8.62$

8.62. $C p A q p$

$8.62 q / N q \times 8.23 p / q, q / p=8.63$

8.63. $C p C q p$

$8.63 q / N p=8.64$

8.64. $C_{p} C N p p$

$8.44 p / r, q \mid A p r, r / q=C 8.62 p / r, q / p-8.65$

8.65. CAqrAqApr

$8.44 p / A q r, q \mid A q A p r, r / p=C 8.65-8.66$

8.66. $C A p A q r A p A q A p r$

$8.51 p / A p A q A p r, q|A A q A p r p, r / A p A q r=C 8.43 q| A q A p r$ $-C 8.66-8.67$

8.67. CApAqrAAqAprp

$8.51 \mathrm{p} / \mathrm{Apr}, q \mid A q A p r, r / p=C 8.62 p / A p r-C 8.42 q / r-8.68$

8.68. $C p A q A p r$

$8.44 q / A q A p r, r / A q A p r=C 8.68-8.69$

8.69. CAAqAprpAAqAprAqApr

$8.51 p / A A q A p r A q A p r, q \mid A q A p r, r_{i}^{\prime} A A q A p r p=C 8.41$

$p / A q A p r-C 8.69-8.70$

8.70. CAAqAprpAqApr

$8.51 p / A A q A p r p, q \mid A q A p r, r / A p A q r=C 8.70-C 8.67-8.71$

8.71. CApAqrAqApr $8.44 p /$ Aqr, $q \mid$ Arq, $r / p=C 8.43 p / q, q / r=8.72$ 
8.72. CApAqrApArq

$8.51 p / A p A r q, q / A r A p q, r / A p A q r=C 8.71 q / r, r / q-$

C8.72-8.73

8.73. CApAqrArApq

$8.51 p / A r A p q, q|A A p q r, r| A p A q r=C 8.43 p / r, q \mid A p q-$

C8.73-8.74

8.74. CApAqrAApqr

$8.51 p / A q A p r, q \mid A q A r p, r / A p A q r=C 8.72 p / q, q / p-$

C8.71-8.75

8.75. CApAqrAqArp

$8.51 p / A r A p q, q \mid A r A q p, r / A p A q r=C 8.72 p / r, q / p, r / q-$

C8.73-8.76

\subsection{CApAqrArAqp}

HiSTOIRE: L'axiomatisation de la logique des propositions fut entreprise par Peano et Frege et perfectionnée par les PM, qui emploient cinq axiomes. Ce nombre fut réduit à quatre par Hilbert, à trois par Łukasiewicz, à un seul par Nicod; ce dernier axiome fut notablement abrégé par Łukasiewicz et Sobocinski.

LitTÉrature: Le système exposé dans ce § est celui de Hilbert-Ackermann, Grundzüge, mais la méthode, qui n'est pas très rigoureuse chez les auteurs, fut remplacée par celle de Łukasiewicz. Les définitions sont basées sur une invention de Sheffer. Pour des systèmes développés voir les traités $(\S 0)$; l'application des méthodes vraiment rigoureuses est encore rare dans la littérature de ce genre.

§ 9. SYSTÈME DE RÈGLES DE LA DÉDUCTION

La théorie exposée dans ce chapitre permet de traduire les lois logiques en règles métalogiques. En pratique les règles, qui indiquent comment on peut procéder dans la déduction, sont de beaucoup plus importantes que les lois, qui énoncent non pas ce qui peut être fait, mais ce qui est. Par exemple, le modus ponendo ponens (6.42) énonce que si $p \supset q$ et $p$, alors $q$, mais ne permet nullement de passer de l'affirmation de , $p \supset q^{\prime \prime}$ et de ,,$p^{\prime \prime}$ à l'affirmation de ., $q "$. Cependant, chaque loi peut être traduite en règle, en vertu de quelques principes peu nombreux. Ces principes seront donnés ici sans justification, qui est du reste très facile.

\subsection{Définitions.}

9.11. „Système 8" pour: „,système exposé au $\S 8$ et dont quelques thèses sont données aux $\S \S 5$ et $6 "$.

9.12. "Loi 8 " pour: ,loi du système 8 ".

9.13. "Règle 9" pour: ,règle obtenue par l'application des principes du $\S 9$ aux lois $8 "$. 


\subsection{Noms des expressions 8:}

9.21. "Négation de $X$ " ou ,non- $X$ " pour: ,groupe composé de , $N$ ' et de $X^{\prime \prime}$.

9.22. "Alternative $X-Y$ " ou ,alternative de $X$ et $Y$ " pour: ..groupe composé de "A', $X$ et $Y^{\prime \prime}$.

9.23. "Implication $X-Y^{\prime \prime}$ ou ,implication de $Y$ par $X "$ pour: ,"groupe composé de ,C', $X$ et $Y^{\prime \prime}$.

9.24. "Disjonction $X-Y$ " ou ,disjonction de $X$ et $Y$ " pour: ,.groupe composé de , $D^{\prime}, X$ et $Y^{\prime \prime}$.

9.25. "Equivalence $X-Y$ " ou ,équivalence de $X$ et $Y$ " pour: ..groupe composé de "E', $X$ et $Y^{\prime \prime}$.

9.26. "Conjonction $X-Y$ " ou ,"conjonction de $X$ et $Y^{\prime}$ pour: ..groupe composé de , $K^{\prime}, X$ et $Y^{\prime \prime}$.

Remarque: les lettres , $X^{\prime \prime}$ et " $Y^{\prime \prime}$ sont des variables auxquelles ne peuvent être substituées que des noms des expressions 8.

\subsection{Règles de traduction.}

9.31. Si $X$ est une loi 8, l'expression composée (1) du nom de $X$, (2) de „peut être affirmé" est une règle 9.

9.32. Si l'équivalence de $X$ et $Y$ est une loi 8, l'expression composée successivement (1) du nom de $X,(2)$ de ,peut être substitué à". (3) du nom de $Y,(4)$ de ,et réciproquement" - est une règle 9.

9.33. Si l'implication $X-Y$ est une loi 8 , l'expression composée successivement (1) de „si", (2) du nom de $X$, (3) de ,.est affirmé". du nom de $Y,(4)$ de „peut être affirmé" - est une règle 9.

9.34. Si l'implication de l'implication $Y-Z$ par $X$ est une loi 8 , l'expression composée successivement (1) de ,si", du nom de $X$, (3) de ,est affirmé et", (4) du nom de $Y$, (5) de ,est affirmé”, (6) du nom de $Z,(7)$ de .,peut être affirmé" - est une règle 9.

9.35. Si l'implication de $Z$ par la conjonction $X-Y$ est une loi 8 , l'expression composée (1) de „si", (2) du nom de $X$, (3) de ,,est affirmé et", (4) du nom de $Y$, (5) de ,est affirmé”, (6) du nom de $Z$, (7) de ,peut être affirmê" - est une règle 9.

\subsection{Exemples de règles 9.}

9.41. La négation de la négation de $X$ peut être substituée par $X(5.12)$. 
9.42. L'alternative $X-Y$ peut être substituée par l'alternative $Y-X(5.22)$.

9.43. La négation de l'alternative $X-Y$ peut être substituée par la conjonction de non- $X$ et non- $Y$ (5.27).

9.44. L'implication $X-Y$ peut être substituée par l'implication non-Y - non-X (5.32).

9.45. L'implication de l'implication $Y-Z$ par $X$ peut être substituée par l'implication de l'implication $X-Z$ par $Y$ (5.33).

9.46. Si $X$ est affirmé, l'alternative $X-Y$ peut être affirmée (6.26).

9.47. Si l'implication $X-Y$ est affirmée et $X$ est affirmé, $Y$ peut être affirmé (6.42).

9.48. Si l'implication $X-Y$ est affirmée et la négation de $Y$ est affirmée, la négation de $X$ peut être affirmée (6.44).

\section{5. Écriture schématique et méthode de Gentzen.}

9.51. Les règles 9 peuvent être représentées schématiquement en traduisant les expressions utilisées en 9.2 et 9.3 comme il suit:

a) "- $X$ " pour: ,,non- $X$ "

, $X+Y$ " pour: ,,alternative $X-Y$ "

",X $X \rightarrow Y$ " pour: ,implication $X-Y$ "

" $X=Y$ " pour: ,équivalence $X-Y$ "

, $X / Y$ " pour: ,disjonction $X-Y$ "

"X×Y" pour: ,conjonction $X-Y$ "

b) ,„म $X$ " pour: , $X$ est affirmé" ou

, $X \propto Y$ " pour: , $X$ peut être substitué à $Y "$

,$\vdash X \vdash Y$ ". pour: ,X est affirmé et $Y$ est affirmé"

.$\stackrel{\vdash X}{\vdash Y}$ pour: „si $X$ est affirmé, $Y$ peut être affirmé”.

9.52. $X+Y \cdot \infty \cdot Y+X$

9.53. $-\cdot X+Y \cdot \infty \cdot-X \times-Y$

9.56. $\frac{X}{X+Y}$

9.57. $\frac{\vdash X \rightarrow Y \vdash X}{\vdash X}$

9.58. $\frac{\vdash X \rightarrow Y \vdash-Y}{\vdash-X}$ 
9.59. En établissant un petit nombre de règles de ce type ( 9.4 ou 9.5 ) on peut construire le système 8 entier, sans axiomes et sans recours à la méthode donnée au $\S 3$.

HistoIRE: La distinction des lois et des règles semble remonter à Husserl. Les travaux les plus notables sur les règles sont ceux de Gentzen (1934) et Jaśkowski (1934). L'élaboration de cette idée est basée ici sur les travaux plus récents des métalogiciens (cf. §1).

LITTÉRATURE: Gentzen, Untersuchungen; Jaskowski, On the rules; Carnap, Foundations; Fẹs, Les méthodes récentes; Popper, New Foundations. 


\section{PRÉDICATS ET CLASSES}

$\S 10$. SyllogistiQue La syllogistique est un système (très simple, mais important dans la pratique) de la logique dite ,des termes", c. à d. dans laquelle apparaissent des variables qu'on ne peut substituer que par des noms, non pas par des propositions.

\subsection{Termes primitifs et règles.}

10.001. Termes primitifs: (a) tous les termes primitifs et définis du système 8 ( $\S 8.12-16)$; (b) variables , ,a", „, $b$ " et ,, $m$ " - auxquelles on ne peut substituer que des noms; (c) opérateurs dyadiques " $A$ " et " $I "$, dont les arguments sont les lettres , $a "$, , $b "$ et , $m "$.

Explication: „a” sera employé pour terme majeur, „, $b$ " pour terme mineur, „ $m$ " pour terme moyen. "A" et "I" (comme aussi "E" et "O" cf. 10.01) ont un sens semblable à celui qu'on leur attribue en logique traditionnelle. Remarquons que " $A$ " et "E" sont ici des opérateurs nominaux, ont donc un sens totalement différent de celui qui fut défini au § 3. Afin d'éviter toute confusion, les opérateurs propositionaux " $A$ " et "E" ne seront pas employés dans ce chapitre.

10.002. Propositions: (1) Toutes les propositions du système 8 , (2) groupes composés de ", $A ",, E^{\prime \prime},, I$ " ou , $O$ " et de deux lettres ,a", , , $b$ " ou , $m ",(3)$ propositions du système 8 dans lesquelles on a substitué des propositions à des variables.

\subsection{Règles: 8.31-32-33.}

10.004. Règle: Une variable ,a", , $b$ " ou , $m$ " peut être substituée par une variable , $a ",,, b "$ ou , $m "$.

Explication: A l'occasion de 8.31 et 10.004 on précise souvent que les variables ne doivent pas être substituées par des noms de classes vides (cf. 15.42). Il faut remarquer à cet égard: (1) que le problème n'a rien à voir avec la structure du système 10 et porte exclusivement sur son interprétation (cf. 7.53); les règles 8.31 et 10.004 ne permettent pas, en effet, de substituer les variables par des expressions autres que les propositions au sens de 10.002 ; (2) que le problème (dit "problème de la classe vide") touche à des questions philosophiques et est fort compliqué. Cf. la littérature.

\subsection{Définitions et axiomes.}

\section{Définitions}

10.01. "Eba" pour „NIba"

10.02. "Oba" pour „NAba" 
Axiomes assumés de la logique propositionelle:

10.03. Cpp

10.04. $\mathrm{CC} p N q \mathrm{C} q \mathrm{~Np}$

10.05. CCpqCNqNp

10.06. CCpqCCqrCpr

10.07. CNNpp

10.08. CCKpqrCKNrqNp

10.09. CCKpqrCCspCKsqr

10.10. CCKpqrCpCqr

10.11. CCKpqrCqCpr

10.12. CCKpqrCCsqCKpsr

10.13. CCKpqrCKpNrNq

10.14. CCKpqrCKqpr

Remarque: Tous ces axiomes sont des thèses démontrables en logique des propositions.

Axiomes spécifiques

10.15. Aaa

(,,tout a est a")

10.16. Iaa

(.,quelque a est $a^{\prime \prime}$ )

10.17. CKAmaAbmAba

(Barbara)

10.18. CKEmalbmOba

(Ferio)

\subsection{Carré logique et conversion.}

Dans les déductions qui suivent la première partie du chiffre (,,10." respectivement ,10.0") sera omise pour simplifier les versets probatifs. Nous commençons par démontrer les lois de la contradiction:

$$
3 \mathrm{p} / E b a \times 1 I I^{\circ}=20
$$

10.20. CEbaNIba

10.21. CNIbaEba

$$
3 \mathrm{p} / \text { NIba } 1 \mathrm{II}^{\circ}=21
$$

10.22. CIbaNEba

$$
4 \mathrm{p} / E b a, q / I b a=C 20-22
$$

$$
5 \mathrm{p} / \mathrm{NIba}, q \mid E b a=\mathrm{C} 21-(1)
$$

(1) CNEbaNNIba

$6 p / N E b a, q / N N I b a, r / I b a=C(1)-C 7 p / I b a-23$

10.23. CNEbalba

10.24. CObaNAba

$$
3 \mathrm{p} / \mathrm{Oba} \times 211^{\circ}=24
$$

$3 \mathrm{p} / \mathrm{Oba} \times 2 I^{\circ}=25$ 
10.25. CNAbaOba

10.26. CAbaNOba

$$
4 \mathrm{p} / \mathrm{Oba}, q \mid \mathrm{Aba}=\mathrm{C} 24-26
$$

$$
5 \mathrm{p} / \mathrm{Nba}, q / \mathrm{Oba}=\mathrm{C} 25-(1)
$$

(1) CNObaNNAba

$$
6 p / N O b a, q / N N A b a, r \mid A b a=C(1)-C 7 p / A b a-27
$$

10.27. CNObaAba

Afin de pouvoir prouver les autres lois du carré logique et celles de la conversion il est nécessaire de déduire d'abord le Datisi:

$8 \mathrm{p} / E b a, q \mid I m b, r / O m a=C 18 \mathrm{~b} / \mathrm{m}, \mathrm{m} / \mathrm{b}-(1)$

(1) CKNOmaImbNEba

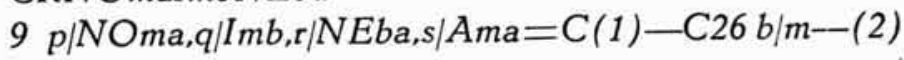

(2) CKAmaImbNEba

$6 \mathrm{p} / \mathrm{KAmaImb}, q \mid \mathrm{NEba}, \mathrm{r} / \mathrm{Iba}=\mathrm{C}(2)-\mathrm{C} 23-30$

10.30. CKAmalmbIba (Datisi)

$10 p / A b b, q / I b a, r / I a b=C 30 a / b, b / a, m / b-C 15 a / b-31$

10.31. CIbalab

$11 \mathrm{p} / \mathrm{Aba}, q|I b b, \tau| I b a=C 30 \mathrm{~m} / \mathrm{b}-\mathrm{C} 16 \mathrm{a} / \mathrm{b}-32$

10.32. CAbalba

$6 \mathrm{p} / \mathrm{Aba}, q \mid \mathrm{Iba}, \tau) \mid \mathrm{Ia}=\mathrm{C} 32-\mathrm{C} 31-33$

10.33. CAbalab

$$
5 p / I a b, q / I b a \times 1 \times 1 a / b, b / a=C 31 a / b, b / a-34
$$

10.34. CEbaEab

$$
5 p / A b a, q / I b a \times 1 \times 2=C 32-35
$$

10.35. CEbaOba

$$
6 p / E b a, q / E a b, r / O a b=C 34-C 35 a / b, b / a-36
$$

10.36. $\mathrm{CEbaOab}$

$$
5 p / A b a, q / I b a=C 32-37
$$

10.37. CNIbaNAba

$$
5 \mathrm{p} / E b a, q / \mathrm{Oba}=\mathrm{C} 35-38
$$

10.38. CNObaNEba

$$
6 \mathrm{p} / \mathrm{Aba}, q / N O b a, r / N E b a=C 26-C 38-39
$$

10.39. CAbaNEba

10.40. CEbaNAba

$$
6 \mathrm{p} / E b a, q|N I b a, r| N A b a=C 20-C 37-40
$$

10.41. CNIbaOba

$$
6 \mathrm{p} / \mathrm{NIba}, q \mid \mathrm{NAba}, \mathrm{r} / \mathrm{Oba}=\mathrm{C} 37-\mathrm{C} 25-41
$$

10.42. CNObalba

$$
6 p / N O b a, q \mid N E b a, r / I b a=C 38-C 23-42
$$


En dehors des lois de la conversion (10.31-33-34-36), des autres regardant l'obversion, la contraposition etc. ont été sont souvent étudiées. On pourait les déduire dans le système en ajoutant deux axiomes et quelques définitions; mais leur importance pratique et théorique étant minime, elles ne seront pas exposées ici.

10.5. Modes syllogistiques.

$6 \mathrm{p} / \mathrm{KAmaAbm,q} \mid \mathrm{Aba}, \mathrm{r} / \mathrm{Iba}=\mathrm{C} 17-\mathrm{C} 32-50$

10.50. CKAmaAbmIba (Barbari)

$12 \mathrm{p} / \mathrm{Ama}, q \mid \mathrm{Imb}, \mathrm{r} / \mathrm{Iba}, \mathrm{s} / \mathrm{Ibm}=\mathrm{C} 30-\mathrm{C} 31 \mathrm{a} / \mathrm{m}-51$

10.51. CKAmalbmIba (Darii)

$9 \mathrm{p} / \mathrm{Ema}, q / \mathrm{Ibm}, \mathrm{r} / \mathrm{Oba}, \mathrm{s} / \mathrm{Eam}=\mathrm{C} 18-\mathrm{C} 34 \mathrm{a} / \mathrm{m}, \mathrm{b} / \mathrm{a}-52$

10.52. CKEamIbmOba (Festino)

$13 \mathrm{p} / \mathrm{Ema}, \mathrm{q} / \mathrm{Iba}, \mathrm{r} / \mathrm{Obm} \times 1=\mathrm{C} 52 \mathrm{a} / \mathrm{m}, \mathrm{m} / \mathrm{a}-(1)$

(1) CKEmaNObmEba

$12 \mathrm{p} / E m a, q|N O b m, r| E b a, s \mid A b m=C(1)-C 26 \mathrm{a} / m-53$

10.53. CKEmaAbmEba (Celarent)

$6 \mathrm{p} / \mathrm{KEmaAbm,q/Eba,r} / \mathrm{Oba}=\mathrm{C} 53-\mathrm{C} 35-54$

10.54. CKEmaAbmOba (Celaront)

$13 \mathrm{p} / \mathrm{Aam}, q \mid A b a, r / A b m \times 2 a / m \times 2=C 17 \mathrm{a} / \mathrm{m}, \mathrm{m} / \mathrm{a}-55$

10.55. CKAamObmOba (Baroco)

$9 \mathrm{p} / E m a, q \mid A b m, r / E b a, s / E a m=C 53-C 34 a / m, b / a-56$

10.56. CKEamAbmEba (Cesare)

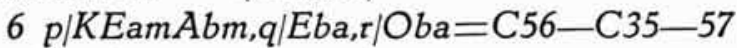

10.57. CKEamAbmOba (Cesaro)

$14 \mathrm{p} / E m a, q \mid A b m, r / E b a=C 53-(1)$

(1) CKAbmEmaEba

$12 p / A a m, q \mid E m b, r / E a b, s / E b m=C(1) a / b, b / a-C 34 a / m-(2)$

(2) CKAamEbmEab

$6 \mathrm{p} / K A a m E b m, q|E a b, r| E b a=C(2)-C 34 a / b, b / a-58$

10.58. CKAamEbmEba (Camestres)

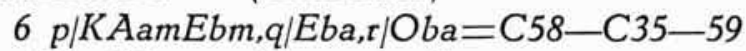

10.59. CKAamEbmOba (Camestrop)

$8 \mathrm{p} / A b a, q|A m b, r| A m a \times 2 b / m \times 2=C 17 b / m, m / b-60$ 10.60. CKOmaAmbOba (Bocardo)

(1) CKImaAmbIab

$14 \mathrm{p} / \mathrm{Amb}, \mathrm{q} / \mathrm{Ima}, \mathrm{r} / \mathrm{Ia}=\mathrm{C} 30 \mathrm{a} / \mathrm{b}, \mathrm{b} / \mathrm{a}-$ (1)

$6 p / K I m a A m b, q / I a b, r / I b a=C(1)-C 31 a / b, b / a-61$

10.61. CKImaAmbIba (Disamis)

$12 p / A m a, q / I m b, r / I b a, s / A m b=C 30-C 32 a / b, b / m-62$ 
10.62. CKAmaAmblba (Darapti)

$12 p / E m a, q / I b m, r / O b a, s / I m b=C 18-C 31 a / b, b / m-63$

10.63. CKEmaImbOba (Ferison)

$12 \mathrm{p} / \mathrm{Ema}, \mathrm{q} \mid \mathrm{Ibm}, \mathrm{r} / \mathrm{Oba}, \mathrm{s} / \mathrm{Amb}=\mathrm{C} 18-\mathrm{C} 33 \mathrm{a} / \mathrm{b}, \mathrm{b} / \mathrm{m}-64$

10.64. CKEmaAmbOba (Felapton)

$12 \mathrm{p} / \mathrm{Eam}, q \mid \mathrm{Ibm}, \tau / \mathrm{Oba}, \mathrm{s} / \mathrm{Imb}=\mathrm{C} 52-\mathrm{C} 31 \mathrm{a} / \mathrm{b}, \mathrm{b} / \mathrm{m}-65$

10.65. CKEamImbOba (Fresison)

$12 \mathrm{p} / \mathrm{Eam}, \mathrm{q} \mid \mathrm{Ibm}, \mathrm{r} / \mathrm{Oba}, \mathrm{s} / \mathrm{Amb}=\mathrm{C} 52-\mathrm{C} 33 \mathrm{a} / \mathrm{b}, \mathrm{b} / \mathrm{m}-66$

10.66. CKEamAmbOba - (Fesapo)

$9 \mathrm{p} / \mathrm{Ima}, q \mid A m b, r / I b a, s / I a m=C 61-C 31 \mathrm{a} / \mathrm{m}, \mathrm{b} / \mathrm{a}-67$

10.67. CKIamAmbIba (Dimaris)

$$
9 \mathrm{p} / \mathrm{Ima}, q \mid A m b, r / \mathrm{Iba}, \mathrm{s} / \mathrm{Aam}=\mathrm{C} 61-\mathrm{C} 33 \mathrm{a} / \mathrm{m}, \mathrm{b} / \mathrm{a}-68
$$

10.68. CKAamAmbIba (Bamalip)

$12 \mathrm{p} / A a m, q \mid E b m, r / E b a, s / E m b=C 58-C 34 \mathrm{a} / b, b / m-69$

10.69. CKAamEmbEba (Camenes)

$6 \mathrm{p} / \mathrm{KAamEmb}, \mathrm{q} \mid \mathrm{Eba}, \mathrm{r} / \mathrm{Oba}=\mathrm{C} 69-\mathrm{C} 35-70$

10.70. CKAamEmbOba (Camenop)

LITTÉRATURE: Le meilleur exposé non-mathématique est: J. N. Keynes, Studies. Axiomatisation: Łukasiewicz, Elementy; Bochenski, Nove Lezzioni, Syllogism. Autres méthodes: Ajdukiewicz, Zolożenia; Black, A new method; Curry, A mathematical treatement; Feys, Logistiek; Greenwood, Les fondements; Miller, The Structure; Moisil, Rech. sur le syllogisme.

\section{§ 11. PRÉdicats MONADIQUES}

Tandis que la syllogistique (cf. $\S 10$ ) analyse la proposition en sujet et prédicat, conçus tous deux comme arguments d'un opérateur dyadique " $A$ ", ,I", "E" ou „O”, la logique des prédicats monadiques conçoit le prédicat comme opérateur et le sujet comme son argument. La quantité est indiquée par une expression spéciale, dite "quantificateur".

\subsection{Définitions.}

11.11. „Constante nominale" pour: ,lettre , $a^{\prime}, b^{\prime}, c^{\prime}$ ou , $d^{\prime}$ ".

11.12. "Variable nominale" pour: ,lettre , $x^{\prime}, y^{\prime}, z^{\prime}$ ou,$t^{\prime}$ ".

11.13. "Opérateur nominal" pour: "lettre , $f^{\prime}, g^{\prime}, h^{\prime}, i^{\prime}$ ' ou , $j^{\prime}$ ".

11.14. "Proposition individuelle" pour: ,expression composée d'un opérateur nominal et de constantes nominales".

11.15. „Matrice" pour: „opérateur nominal suivi de variables nominales".

11.16. , $f x$ " ou , $f(x)$ " pour: , $f$ de $x "$.

Explication: , $f$ de $x^{\prime \prime}$ signifie que la propriété $f$ convient à $x$. 


\subsection{Quantificateurs.}

11.21. „Quantificateut universel” pour: „variable ou variables séparées par des virgules, entre parenthèses rondes (écriture péanorussellienne) ou précédées de,$\Pi^{\prime}$ (écriture Łukasiewicz), le tout placé avant une matrice".

$$
\begin{aligned}
& \text { Exemples: „(x)", , }(y) ", \ldots(x, y) ", \ldots(x, z, y) " \\
& \text { „Пx", ,Пy", „Пxy", „Пxzy" }
\end{aligned}
$$

11.22. „Quantificateur existentiel” pour: , ,E' suivi d'une ou de plusieurs variables séparées par des virgules, le tout entre parenthèses rondes (écriture péano-russellienne) ou,$\Sigma^{\prime \prime}$ suivi d'une ou plusieurs variables (écriture Łukasiewicz), le tout placé devant une matrice".

Exemples: „(Ex)", , $(E x)^{\prime \prime}, \ldots(E x, y) "$.

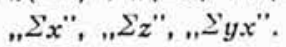

N.b.: dans les $P M$ le "E" contenu dans le quantificateur est renversé.

11.23. „Quantificateur” pour: „quantificateur universel ou quantificateur existentiel".

11.24. , $(x)^{\prime \prime}$ ou " $\Pi x "$ pour: ,,pour tout $x "$.

Exemple: , , $(x) f x^{\prime \prime}$ se lit: „pour tout $x:\left\{\right.$ de $x^{\prime \prime}$ p. ex.: ,pour tout $x$, fume de $x^{\prime \prime}$ c. à d.: „tout $x$ fume".

11.25. ., $(E x) "$ ou , $\Sigma x^{\prime \prime}$ pour: ,,il y a au moins un $x$ tel que:".

Exemple: , $(E x) f x "$ se lit: ,il y a au moins un $x$ tel que $f$ de $x^{\prime \prime}$ p. ex.: ,il y a au moins un $x$ tel que: fume de $x^{\prime \prime}$, c. à d.: ,il existe un être fumant".

\subsection{Variables libres et liées.}

11.31. „Variable libre ou ,réelle” pour: „variable contenue dans une matrice non précédée d'un quantificateur qui comprend une lettre isomorphe à elle-même".

Exemple: la variable , $x "$ dans: ,f $f x \supset g x^{\prime \prime}$.

11.32. „Variable liée" ou ,apparente” pour: „variable contenue dans une matrice qui est précédée d'un quantificateur contenant une lettre isomorphe à cette variable".

Exemple: ,,$x^{\prime \prime}$ est une variable liée dans:,$(x) \cdot\{x \supset g x "$, car la matrice en question est précédée du quantificateur,$(x)$ ".

11.33. Règle: Une variable liée ne peut être substituée.

11.34. „X est lié par le quantificateur $Y$ " pour: , $X$ est une variable 
qui fait partie d'une matrice précédée par $Y$, et $Y$ contient une lettre isomorphe à $X^{\prime \prime}$.

11.35. Règle: Aucune variable ne peut être liée par plus d'un quantificateur.

11.36. "Cloture universelle de $X$ " ou ,universalisation de $X$ " pour: ,expression isomorphe à $X$, précédée des quantificateurs universels liant toutes les variables de $X$ (où $X$ est une matrice)".

11.37. "Cloture existentielle de $X$ " ou ",particularisation de $X$ " pour: ,expression isomorphe à $X$, précédée des quantificateurs existentiels liant toutes les variables de $X$ (où $X$ est une matrice)".

11.38. "Clôture de $X$ " ou ,généralisation de $X$ " pour: ,universalisation où particularisation de $X^{\prime \prime}$.

Exemples:,$(x) f x "$ est une clôture universelle (une universalisation) de " $f x^{\prime \prime}$; . $(E x y) \cdot\{x \cdot g y "$ une clôre existentielle (particularisation) de ., $\{x \cdot g y "$. Les deux sont des généralisations.

Explication: une clôture n'est pas une matrice, mais une proposition: ses variables ne peuvent plus être substituées; elle a une valeur, tandis que la matrice n'en a pas. - Les lois 8 devraient toutes être précédées de quantificateurs; si ils sont omis c'est parce qu'en logique des propositions les quantificateurs sont toujours universels et on ne risque aucune équivoque. On peut cependant construire, même dans ce domaine, une théorie avec des quantificateurs existentiels.

11.39. „Implication formelle" pour: ,clôture universelle d'une expression composée d'une matrice, de, $\supset$ ' et d'une autre matrice, les variables de la première matrice étant isomorphes à celles de la deuxième".

Exemple: ,. $(x) \cdot f x \supset g x "$.

Explication. L'implication formelle (avec des opérateurs constants) correspond à peu près à la proposition universelle affirmative du langage courant: „Tout logicien est un fumeur de pipe" se transcrit: ,. $(x)$. logicien $(x) \supset$ fumeur de pipe $(x)^{\prime \prime}$. - Il y a donc lieu de distinguer trois implications: l'implication matérielle (3.5), l'implication formelle (11.39) et l'implication inférentielle (7.48).

HISTOIRE: L'idée d'employer constamment le quantificateur et l'invention du symbolisme est due à Frege (Begriffschrift, 1879). Ce fut le pas décisif vers la formation de la logique formelle dans son état actuel. La théorie reçut récemment de très considérables développements dans la logique dite „combinatoire", fondée par Schönfinkel et Curry (cf. § 25.2).

LITTÉRATURE: La théorie classique des prédicats et l'énumération des lois se trouvent dans tous les bons traités, en particulier: PM ${ }^{\star} 9-{ }^{\star} 10$.

\section{§ 12. LOIS DES PRÉDICATS MONADIQUES}

les théories logiques qui suivent.

Ce chapitre contient, sans démonstration, les lois les plus fondamentales de la logique des prédicats monadiques; ces lois forment la base de toutes 
Dans certaines lois de ce chapitre et des suivants nous utiliserons des groupes de points plus nombreux qu'il est nécessaire, afin de faciliter la compréhension.

\subsection{Principe méthodique.}

Toutes les lois des prédicats monadiques peuvent être déduites en partant des lois 8 et des deux définitions suivantes:

12.11. ", $(x)\{x "$ pour: , $\{a \cdot f b \cdot f c \cdot f d \cdot \ldots "$

12.12. , $(E x)\{x "$ pour: , $\{a \vee f b \vee f c \vee f d \ldots "$

le nombre des arguments étant supposé indéfini.

Explication: 12.11 supposé que „tout $x$ possède la propriété $f$ " signifie la même chose que ,a possède la propriété $E$, et $b$ la possède, et $c$ etc."; 12.12 dit que „quelque $x$ possède la propriété $l$ " signifie: „a possède la propriété $f$, ou $b$ la possède, ou $c$ etc.". Ces définitions rencontrent de très sérieuses difficultés logiques (car la notion du „etc" est fort compliquée et ne saurait être définie sans l'aide des expressions du type utilisé ici) mais sont commodes en pratique. Ce qui est plus, on peut déduire la grande majorité des lois des prédicats en partant de définitions plus restreintes encore:

,(x)fx" pour: ,fa. $f b^{\prime \prime}$

,(Ex) $f x "$ pour: , ,fa $\vee f b "$

En effet, toutes les propositions déduites de ces définitions à l'aide des règles 9 sont vraies, si longtemps qu'on n'introduit pas de postulats individuels.

\subsection{Négation des prédicats monadiques quantifiés.}
12.21. $(x) f x \equiv \sim(E x) \sim f x$ $E \Pi x\{x N \Sigma x N[x$
12.22. $\sim(x) f x \equiv(E x) \sim f x$ $E N \Pi x\{x \Sigma x N[x$
12.23. $(x) \sim\{x \equiv \sim(E x) f x$ $E \Pi x N\{x N \Sigma x\{x$
12.24. $\sim(x) \sim\{x \equiv(E x)\{x$ $E N \Pi x N\left\{x \sum x\{x\right.$

12.25. Règle: En niant tous les quantificateurs et les matrices et en substituant les quantificateurs universels par des quantificateurs existentiels, et réciproquement, on ne change pas la valeur d'une proposition.

\subsection{Lois fondamentales}
12.31. $(x) f x \supset f y$
12.32. $f y \supset(E x) f x$
$C \Pi x f x f y$
12.33. $(x)\{x \supset(E x)\lceil x$
Cfy $\Sigma x f x$
$C \Pi x\{x \Sigma x\{x$

Explication: 12.31 signifie: „si $f$ (universellement) de tout $x$, alors $t$ de $y$ "; cette loi se déduit de 12.11 en vertu de 6.27 .12 .32 signifie: „si $\ell$ de $y$, alors il y a (au moins un) $x$ tel que $t$ de $x "$; elle se déduit de 12.12 en vertu de 6.26 .12 .33 est la loi de subalternation bien connue, qui s'obtient en vertu de la loi du syllogisme (6.31 ss) en partant de 12.31 et 12.32 . 
12.34. Les lois $12.31-32$ ajoutées comme axiomes au système 8 suffisent (avec des règles et des définition nouvelles) pour établir le système axiomatique des prédicats.

\subsection{Règles de la déduction.}

12.41. Le quantificateur universel placé au commencement d'une proposition affirmée peut être omis si il se rapporte à l'ensemble des expressions qui le suivent dans cette proposition. (12.31).

12.411. Si la cloture universelle de la matrice $X$ est affirmée, l'expression formée par la substitution des variables de $X$ par des constantes, peut être affirmée. (12.31).

Exemple: soit l'affirmation "tout $x$ est mortel" c. à d. ., $(x)$ mortel $x$ "; en vertu de 11.31 la proposition „Pierre est mortel" peut être affirmée.

12.42. Si la matrice $X$ est affirmée, la clôture existentielle de $X$ peut être affirmée. (12.32).

12.421. Si la proposition individuelle $X$ est affirmée, la clôture existentielle de la matrice formée par la substitution des constantes de $X$ par des variables, peut être affirmée. (12.32).

Exemple: soit la proposition individuelle ,Pierre fume" c. à d. „fume (Pierre)"; en vertu de $11.32, \ldots(E x)$ fume $x "$, c. à d. la proposition ,il y a un $x$ qui fume" peut être affirmée.

12.43. Si la clôture universelle de $X$ est affirmée, la clôture existentielle de $X$ peut être affirmée. (12.33)

12.44. Si la matrice $X$ est affirmée, la clôture universelle de $X$ peut être affirmée.

Explication: 12.44 n'est pas fondée, comme le sont 12.41-42-43 sur une loi; elle peut être cependant justifiée soit par l'application de la méthode 12.1, soit par la considération suivante: ,,$f x^{\prime \prime}$ affirme que $t$ convient à un $x$ quelconque; donc, $\hat{E}$ convient à tout $x$; ce qui est exprimé par " $(x) f x "$.

\subsection{Lois analogues.}

12.51. „X est une expression analogue (12.5) à $Y$ " pour: , $X$ est une expression formée de $Y$ par la substitution de , $f x^{\prime}$ à , $p^{\prime},, g x^{\prime}$ à , $q^{\prime}$, ,$h x^{\prime}$ à $, t^{\prime},, i x^{\prime}$ à $, s^{\prime},, j x^{\prime}$ à,$t^{\prime}$ et précédée de,$(x)^{\prime}$ ou de , $\Pi x^{\prime}{ }^{\prime \prime}$.

12.52. Toute expression analogue (12.5) à une loi 8 est une loi.

12.53. $(x) \cdot\{x \equiv\{x$ $\Pi x E\{x\{x$

Principe d'identité pour les prédicats (cf. 5.11)

12.54. $(x) \cdot\{x \mid \sim\{x$ $\Pi x D\{x N\{x$ 
12.55. $(x): \sim \cdot\{x \cdot \sim\{x$

$\Pi x N K\{x N\{x$

Principe de non-contradiction pour les prédicats (cf. 6.11-12).

12.56. $(x) \cdot\{x \vee \sim\{x$

$\Pi x A[x N\{x$

Principe du tiers-exclu pour les prédicats (cf. 6.13).

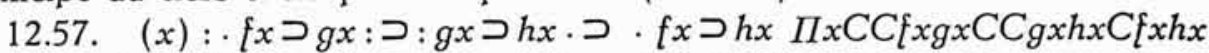

Principe du syllogisme pour les prédicats (cf. 6.32).

12.58. $(x): f x \supset g x \cdot f x \cdot \supset \cdot g x$

$\Pi x C K C f x g x f x g x$

Modus ponendo ponens pour les prédicats (cf. 6.42).

\subsection{Lois du mouvement des quantificateurs.}

12.61. $(x) \cdot f x \cdot g x \cdot \equiv \cdot(x)\{x \cdot(x) g x$

$E \Pi x K f x g x K \Pi x f x \Pi x g x$

Exemple: si tout homme est un mammifère et un bipède, tout homme est un mammifère et tout homme est un bipède; l'inverse est aussi vrai.

\subsection{2. $(E x) \cdot f x \cdot g x \cdot \supset \cdot(E x) f x \cdot(E x) g x \quad C \Sigma x K\{x g x K \Sigma x\{x \Sigma x g x$}

Exemple: s'il y a un homme qui est logicien et fumeur de pipe, il y a un homme qui est logicien et il y a un homme qui est fumeur de pipe; l'inverse n'est pas vrai.

12.63. $(E x) \cdot\left[x \vee g x . \equiv \cdot(E x)\left\{x \vee(E x) g x \quad E \Sigma x A\left\{x g x A \Sigma x\left\{x \sum x g x\right.\right.\right.\right.$ 12.64. $(x)\{x \cdot v \cdot(x) g x: \supset:(x) \cdot\{x \vee g x \quad C A \Pi x\{x \Pi x g x \Pi x A\{x g x$

Exemple: si toutes les locomotives sont grandes ou toutes les locomotives sont petites, toutes les locomotives sont grandes ou petites. L'inverse n'est pas vrai.

12.65. $(x) \cdot f x \supset g x: \supset:(x) f x \cdot \supset \cdot(x) g x$

$C \Pi x C\{x g x C \Pi x\{x \Pi x g x$

12.66. $(x) \cdot f x \equiv g x: \supset:(x) f x \cdot \equiv \cdot(x) g x$

$C \Pi x E\{x g x E \Pi x\{x \Pi x g x$

L'inverse n'est pas vrai.

12.71. $(x) \cdot f x \vee p: \equiv:(x) f x \cdot \vee \cdot p$

12.72. $(E x) \cdot\{x \vee p: \equiv:(E x)\{x \cdot \vee \cdot p$

$E \Pi x A\{x p A \Pi x[x p$

12.73. $(x) \cdot\{x \supset p: \equiv:(E x)\{x \cdot \supset \cdot p$

12.74. $(E x) \cdot\{x \supset p: \equiv:(x)[x \cdot \supset \cdot p$ $E \Sigma x A[x p A \Sigma x\{x p$ $E \Pi x C\{x p C \Sigma x\lceil x p$ $E \Sigma x C\{x p C \Pi x\{x p$

Nous avons par contre:

12.75. $(x) \cdot p \supset f x: \equiv: p \cdot \supset \cdot(x) f x$

12.76. $(E x) \cdot p \supset f x: \equiv: p \cdot \supset \cdot(E x) f x$

Explication: ces dernières lois qui semblent paradoxales cessent de l'être quand on considère $12.21,12,22$ et 5.311 .

12.8. Lois syllogistiques.

12.81. (x) $f x \supset g x:(x) \cdot h x \supset f x: \supset:(x) \cdot h x \supset g x$

CKח $\operatorname{Cf} x g x \Pi x C h x f x \Pi x C h x g x$

(Cf. 10.17) 
12.82. $(x) \cdot\{x \supset g x:(E x)\{x: \supset:(E x) g x$

CKח $\Pi \mathrm{C}\{x g x \Sigma x\{x \Sigma x g x$

12.83. $(x) \cdot\{x \supset g x:(E x) \sim g x: \supset:(E x) \sim f x$

CKП $\mathrm{x} C\{x g x \Sigma x N g x \Sigma x N\{x$

12.84. $(x) \cdot\{x \vee g x:(E x) \sim\{x: \supset:(E x) g x$

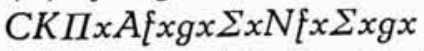

12.85. $(x) \cdot\{x \mid g x:(E x)\{x: \supset \cdot(E x) \sim g x$

$C K \Pi x D f x g x \Sigma x\{x \Sigma x N g x$

12.86. $(x) \cdot\{x \equiv g x:(E x)\{x: \supset:(E x) g x$

$C K \Pi x E\{x g x: \Sigma x\{x \Sigma x g x$

12.87. $(x) \cdot\{x \equiv \sim g x:(E x) f x: \supset:(E x) \sim g x$ $C K \Pi x E f x N g x \Sigma x f x \Sigma x N g x$

12.9. Lois avec constantes.

12.91. $(x) \cdot\{x \supset g x:\{a: \supset: g a$ CKПxCfxgxfaga

Explication: cette loi est un autre forme de Barbara (10.17) à côté de 12.81. La différence entre 12.81 et 12.91 est considérable.

12.92. $(x) \cdot\{x \supset g x: \sim g a: \supset: \sim\{a$ $\mathrm{CK} \Pi x \mathrm{C}\{x g x \mathrm{NgaNfa}$

Explication: 12.92 est une autre forme de Baroco (10.55); cf. 10.83.

12.93. $(x) \cdot f x \vee g x: \sim f a: \supset: g a$

CKחxAfxgxNfaga

(Cf. 12.84)

12.94. $(x) \cdot f x \mid g x: f a: \supset: \sim g a$

CKMxD $\{x g x f a N g a$

12.95. $(x) \cdot f x \equiv g x:\{a: \supset: g a$

CKMxE[xgxfaga

12.96. $(x) \cdot\{x \equiv \sim g x:\{a: \supset: \sim g a$

CKMxE\{xNgxfaNga

12.97. La théorie exposée dans ce chapitre porte le nom de „calcul des prédicats $d u$ premier ordre" ou ,calcul inférieur". Il existe, à côté de lui un calcul supérieur, dans lequel on considère les prédicats des prédicats et où les prédicats eux-mêmes sont quantifiés. Mais ce calcul, quoi qu'il soit indispensable pour l'analyse, n'a pas encore été développé formellement.

HISTOIRE ET LITTÉRATURE: v. § 11.

$\S$ 13. PRÉDICATS DYADIQUES $\begin{aligned} & \text { Dans la vie quotidienne et surtout dans les } \\ & \text { sciences, on emploie souvent des prédicats }\end{aligned}$ dyadiques (p. ex. „Isidore fume la pipe"), et, ce qui est plus important, avec les 
deux arguments quantifiés, comme p. ex. dans la proposition ,il y a des hommes qui aiment tous les êtres vivants". La théorie de ces prédicats s'obtient facilement à partir des données du $\S 12$.

\subsection{Définitions.}

13.11. ", $f(x, y)$ " pour: , $f$ de $x$ et $y "$.

13.12. ", $(x, y) f(x, y)$ " pour: , $(x) \cdot(y) \mathcal{f}(x, y)$ " "Пxyfxy" pour: "П $\Pi x \Pi y\{x y "$

13.13. , $(E x, y) \mathcal{E}(x, y)$ " pour: ,. $(E x) \cdot(E y) \mathcal{E}(x, y) "$ ,$\Sigma x y\{x y$ " pour: , $\Sigma x \Sigma y\{x y "$

13.14. " $(x)(E y)\{(x, y)$ " pour: ,. $(x) \cdot(E y)\{(x, y)$ "

13.15. " $(E x)(y) f(x, y) "$ pour: ,. $(E x) \cdot(y) \mathcal{F}(x, y) "$

\subsection{Lois du mouvement des quantificateurs.}
13.21. $(x, y)\{(x, y) \cdot \equiv \cdot(y, x)\{(x, y)$
13.22. $(E x, y) f(x, y) \cdot \equiv \cdot(E y, x) f(x, y)$

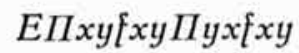
$E \Sigma x y[x y \Sigma y x[x y$

13.23. Règle. Si les quantificateurs d'une proposition liant les zarguments du même opérateur nominal sont tous universels ou tous existentiels, on peut changer leur ordre sans changer la valeur de la „proposition.

13.24. $(E x)(y)\{(x, y) \cdot \supset \cdot(y)(E x) f(x, y) \quad C \Sigma x \Pi y\{x y \Pi y \Sigma x\{x y$

Explication: cette loi n'est qu'une implication, non pas une équivalence, car son inverse:

$$
(x)(E y) f(x, y) \text {. . }(E y)(x) f(x, y)
$$

est fausse, comme on peut le voir dans l'exemple suivant. Soit , $f(x, y)$ " une abréviation de „x ressemble à $y "$; alors „ $(x)(E y) f(x, y) "$ se lit: „pour tout $x$ il $\mathrm{y}$ a au moins un $y$ tel que $x$ ressemble à $y$ " c. à d.: , chaque chose a quelque chose qui lui ressemble"; mais " $(E y)(x) f(x, y)$ " se lit: ,il existe au moins un $y$ tel que, pour tout $x, x$ ressemble à $y^{\prime \prime} \mathrm{c}$. à d.: ,il $\mathrm{y}$ a au moins une chose qui ressemble à toutes les choses". La première proposition semble être vraie, tandis que la deuxième est manifestement fausse.

\subsection{Lois analogues.}

13.31. "X est une expression analogue (13.3) à $Y$ " pour: , $X$ est une expression formée par la substitution, dans $Y$, de tous les ', $x^{\prime}$ par ., $(x, y)^{\prime}$ dans les arguments et par , $x, y$ ' dans les quantificateurs".

13.32. Toute expression analogue (13.3) à une loi du $\S 12$, est une loi.

13.34. Règle: En construisant une définition semblable à 13.31 pour les opérateurs triadiques et supérieurs, on peut former une règle du même genre qui permet d'établir des lois analogues pour ces quan:tificateurs. 
HISTOIRE: La logique des prédicats dyadiques apparait chez Frege et Peano. C'est une des acquisitions les plus importantes de la logique mathématique.

LITTÉRATURE: PM *11 et les autres traités.

\section{$\S$ 14. IDENTITÉ ET DESCRIPTION Dans le présent chapitre nous unissons} deux théories assez différentes. Celle de l'identité sert de préambule à la logique des classes et joue un rôle considérable dans les développements ultérieurs de la logique; elle traite de la notion,$x$ est la même chose que $y^{\prime \prime}$. - La théorie de la description est une sorte de grammaire logique de l'article singulier "le". Elle permet de formuler et de traiter axiomatiquement les expressions du type ,le $x$ tel que...". Son importance dans toute application de la logique est aussi très grande.

\subsection{Identité.}

14.11. , ,x $x=y$ " pour: , $x$ est identique à $y "$.

Explication: l'identité peut être définie comme suit:

$$
\text { " } x=y \text { " pour: " }(f) \cdot f x \supset f y^{\prime \prime}
$$

mais cette définition, basée sur le principe des indiscernables de Leibniz et dite "thèse d'extensionalité", entraine de graves difficultés dans l'application de la logique aux autres domaines. Pour cette raison il est préférable d'introduire l'identité comme terme primitif (non-défini).

14.12. , $x \neq y "$ pour: , $\sim \cdot x=y "$.

Explication: 14.12 définit la diversité.

14.13. $(x) \cdot x=x$

Explication: 14.13 est une autre forme du principe d'identité (cf. 5.11 et 12.53).

14.14. $(x, y): x=y \cdot \supset \cdot y=x$

14.15. $(x, y, z): x=y \cdot y=z \cdot \supset \cdot x=z$

Explication: ces trois lois formulent les caractères principaux de l'identité: celle-ci est réflexive (14.13), symétrique (14.14) et transitive (14.15). Cf. 22.

14.16. , „xIy" pour: , ,x $=y "$

14.17. ",xJy" pour: , $x \neq y "$

14.18. $(x, y): x=y \cdot \supset \cdot(f) \cdot f x \supset f y$

Explication: si $x$ et $y$ sont identiques, $y$ possède tous les prédicats que possède $x$.

\subsection{Descriptions.}

14.21. „Description" pour: „matrice à une variable, précédée de $r^{\prime}$ et de l'isomorphe de cette variable entre parenthèses". 14.22. „( $(x)(f x)$ " pour: ,le $x$ tel que $\{x "$. Description.

Exemples: „l'auteur de Quo Vadis", „le carré de 9", „le premier roi de Hongrie”, "l'automobile de Jean". 
14.23. ,E! $(\imath x)(f x) "$ pour: , ,(Eb) $(x): f x . \equiv \cdot x=b: f b^{\prime \prime}$

Explication: „E! $(f x)(f x)^{\prime}$ signifie d'après notre définition que la chose décrite par , $(1 x)\left([x)^{\prime \prime}\right.$ existe et qu'elle est unique; elle existe, car nous avons ., $(E b)$ "; elle est unique, car, d'après la définition, tout $x$ qui possède la propriété $f$ est identique à ce $b$. Décrire par le mot "le" une classe qui contient plus d'un élément n'a pas de sens; p. ex. l'expression ,le général anglais", donnée sans autre précision, est privée de sens, car il y a plus d'un général anglais.

\subsection{4. $g[(1 x)(f x)] \cdot \supset \cdot E !(1 x)(\{x)$}

Explication: l'affirmation que la chose décrite possède une propriété implique son existence.

Exemple: „l'auteur de la Divine Comédie était italien" implique que cet auteur existait; ,l'automobile de Jean est une Vauxhall" implique qu'il y a une automobile de Jean.

Histolre: La théorie de la description, connue de Frege et de Peano, fut surtout élaborée par Russell. Elle implique des problèmes philosophiques fort difficiles qui ne sont pas encore totalement élucidés. - Créée par Leibniz, la théorie de l'identité fut, par Peano, achevée jusque dans les détails.

LITTÉRATURE: $\S 14.1: P M{ }^{\star} 13$; pour les difficultés inhérentes à la définition Leibnizienne: PM I, p. 659 ss; Ajdukiewicz, Anwendbarkeit. — §14.2: PM *14; Russell, On Denoting; Moore, Russell's Theory; point de vue différent: HilbertBernays, Grundlagen; Quine, Mathematical Logic.

$\S 15$. Classes La théorie des prédicats considère la compréhension des termes (opérateurs); c'est, au contraire, leur extension qu'examine la théorie des classes. Celle-ci est donc parfaitement analogue à celle-là. Nous donnons ici la doctrine péano-russellienne; il convient toutefois de signaler qu'en plus de cette théorie il en existe une autre, récente, due à Leśniewski ("ontologie"), qui ne reconnait pas de classe vide et se base sur un seul terme primitif „est". Le présent chapitre donne les définitions; le $§ 16$ exposera quelques lois importantes.

\subsection{Définitions fondamentales.}

15.11. , , $\hat{x}\{f x\}$ " pour: ,les $x$-es tels que: $f x^{\prime \prime}$.

Exemples: „les $x$-es tels que: $x$ fume la pipe” c. à d. „les fumeurs de pipe”; "les $x$-es tels que: $x$ habite Londres" c. à d. "les habitants de Londres".

Explication: 15.11 définit la classe par une fonction propositionelle; cette opération porte le nom "d'abstraction": la classe des fumeurs de pipes est une abstraction de la fonction , $x$ fume la pipe".

15.111. , , $\lambda x f x$ " pour: , $\hat{x}\{f x\} "$

Explication: L'expression ", $\lambda$ " qui porte le nom $d$ "opérateur lambda" est souvent substituée, ces deniers temps surtout, à l'x avec accent circonflexe des $P M$. 
15.12. "Cls" pour: , ,h $\{(E f) \cdot a=\hat{x}(\{x)\} "$

Explication: c'est la définition de la classe de toutes les classes: elle est composée de tous les $\alpha$ tels que $a=\hat{x}(f x)$ pour un $t$ quelconque c. à d. selon 15.11 , des classes.

15.13. , ,y $\varepsilon \hat{x}(f x) "$ pour: , $\{y "$.

Explication: dire: „y est un élément de la classe de ces $x$ pour lesquels vaut , $\left\{x^{\prime}\right.$ " revient à dire ,ffy".

Exemple: chaque Suisse est un élément de la classe des Suisses, chaque homme qui fume la pipe en est un de celle des fumeurs, chaque montagne des Alpes un élément de la classe dite „Alpes" etc.

15.14. ,,$x \sim \varepsilon \alpha "$ pour: , $\sim \cdot x \varepsilon \alpha^{\prime \prime}$

15.15. , ,x, $y \varepsilon \alpha^{\prime \prime}$ pour: , $x \varepsilon \alpha \cdot y \varepsilon \alpha "$.

\subsection{Relations entre les classes.}

15.21. ,- $-\alpha$ " pour: , , $\hat{x}(x \sim \varepsilon \alpha)$ ". Classe complémentaire de $\alpha$.

Explication: La classe complémentaire de $a$ comprend comme éléments toutes les choses qui ne sont pas des éléments de $\alpha$. Exemple: la classe complémentaire de la classe des éléphants est la classe des non-éléphants. Il est évident que le monde est plein de non-éléphants.

15.22. , , $\alpha \cup \beta$ " pour: , $\hat{x}\left(x_{\varepsilon} \alpha \cdot \mathrm{v} \cdot x \varepsilon \beta\right)$ ". Somme logique des classes. 15.23. ., $\alpha \cap \beta$ " pour: , $\hat{x}\{x \varepsilon \alpha \cdot x \varepsilon \beta\} "$. Produit des classes.

15.24.,$\alpha \| \beta$ " pour: , $\hat{x}\{x \varepsilon \alpha \cdot \mid \cdot x \varepsilon \beta\}$ ". Disjonction des classes.

Exemple: soit $\alpha$ la classe des fumeurs de pipe et $\beta$ celle des logiciens. Dans ce cas $\alpha \cup \beta$ est la classe de tous ceux qui sont soit des fumeurs de pipe, soit des logiciens; $\alpha \cap \beta$ est la classe des logiciens qui fument la pipe; $\alpha \| \beta$, celle de ceux qui ne sont pas à la fois logiciens et fumeurs de pipe.

15.25. , , $\alpha \subset \beta^{\prime \prime}$ pour: , , $(x): x \varepsilon \alpha \cdot \supset \cdot x \varepsilon \beta "$. Inclusion des classes. 15.26.,$\alpha=\beta$ " pour: , , $(x): x \varepsilon \alpha$. $\equiv \cdot x \varepsilon \beta "$. Egalité des classes.

Exemples: la classe des fumeurs de pipe est incluse dans la classe des fumeurs; celle des citoyens français de 21 ans et plus est égale à la classe des hommes qui ont le droit de vote en France. A noter que , $\alpha \subset \beta^{\prime \prime}$ et , $\alpha=\beta^{\prime \prime}$ sont des propositions, tandis que , $\alpha \cup \beta$ " et , $\alpha \cap \beta$ " des noms de classes.

15.27. Règle des points: un groupe de points, placé près d'un opérateur de vérité, a un rang supérieur au groupe de points placé près d'un des opérateurs définis en $15.21-26$. 


\subsection{Représentation graphique.}

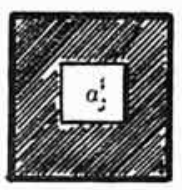

$-a$

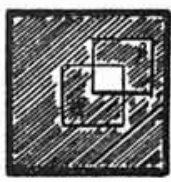

$a \| \beta$

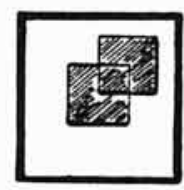

$a \cup \beta$

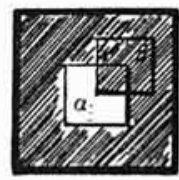

$a \subset \beta$

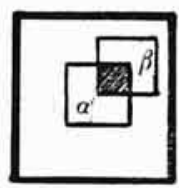

$a \cap \beta$

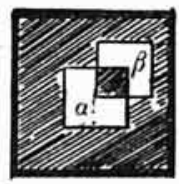

$a=\beta$

\subsection{Existence.}

15.41. „," pour: ,,$\hat{x}(x=x) "$. Classe universelle.

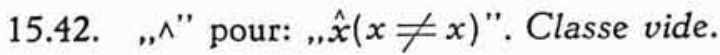

Explication: La classe universelle est la classe de tous les $x$ qui sont identiques à eux-mêmes c. à d. de tous les $x$ en général. La classe vide est la classe des $x$ qui ne sont pas identiques à eux-mêmes, c. à d. des objets qui n'existent pas.

Exemples: la classe des rois Suisses, des femmes de Copernic, des pères d'Adam, des automobiles d'un homme qui r'a pas de voiture, est la classe vide.

15.43. , , ,

Explication:,$\exists ! \alpha^{\prime \prime}$ signifie que la classe $\alpha$ n'est pas une classe vide, c. à d. qu'il y a des éléments dans $\alpha$. Il faut distinguer l'existence des éléments d'une classe et l'existence de la classe elle-même: même dans le cas où $\sim \exists$ ! $\alpha$, c. à d. oủ $\alpha=\wedge$, la classe $\alpha$ existe, tout en étant vide.

\subsection{Les sens du mot, „est".}

15.51. Le mot français „est” (et les mots semblables dans les autres langues européennes) comporte deux groupes de sens très différents: sens existentiels et sens copulatifs.

15.52. Il y a, entre autres, deux sens existentiels du mot ,est": (1) existence d'un objet décrit (,E!", 14.23), (2) non-vacuité d'une classe (,,B!", 15.43); cette dernière est définie à l'aide du quantificateur existentiel $\left(.,(E x)^{\prime \prime}, 11.22 ; 11.25\right)$. 
15.53. Il y a, entre autres, trois sens copulatifs du mot „est": (1) appartenance d'un élement à une classe $\left(, \varepsilon^{\prime \prime}, 15.13\right)$; cette notion est définie à l'aide d'une matrice (, $\left\{x^{\prime \prime}, 11.15 \mathrm{~s}.\right)$; (2) inclusion d'une classe dans une autre (,С", 15.25); (3) identité (,=", 14.11).

\subsection{Classes unitaires et binaires.}

15.61. , $[x] "$ pour: , , $\hat{y}(y=x)$ ". Classe unitaire.

Explication: la classe $[x]$ est la classe qui a pour élément unique $x$; ex.: la classe unitaire des lunes terrestres. Elle se distingue malgré cela de son élément, car elle possède des propriétés que l'élément ne possède pas: p. ex. elle contient un élément.

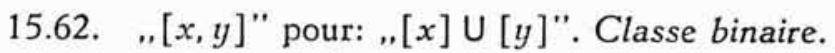

15.63. "1" pour: ,, $\hat{a}\{(E x) \cdot a=[x]\} "$. Unité cardinale.

Explication: l'unité cardinale est la classe de toutes les classes unitaires. En effet, quand je dis que j'ai un crayon, je qualifie non pas le crayon, mais la classe de mes crayons; ceci est particulièrement clair quand on passe aux nombres supérieurs: on ne peut attribuer un nombre qu'à une classe (Frege).

\subsection{4. "2" pour: , $\hat{\alpha}\{(E x, y): \alpha=[x, y] \cdot x \neq y\} "$.}

Explication: 2 est la classe de toutes les classes binaires, dont les éléments ne sont pas identiques entre eux.

Histoire: Boole est le véritable créateur de la logique des classes. Son ,algèbre logique" fut la première partie de la logique mathématique complètement élaborée. Il employait des signes (., $\times "$, "," etc.) et des opérations semblables à celles des mathématiques. Frege et après lui Peano ont défini la classe en partant des fonctions propositionnelles.

LitTÉRATURE: PM ${ }^{\star} 20,{ }^{*} 22,{ }^{\star} 24$; pour l'algèbre de la logique: Lewis, Survey. Élaboration moderne: Moisil, Recherches sur l'algèbre; Système de Leśniewski: Uber die Grundlagen.

\section{$\S 16$. Calcul des classes}

\subsection{Lois analogues.}

16.11. "X est une expression analogue (16.11) de $Y$ " pour: , $X$ est une expression formée par la substitution de , $\left[x^{\prime}\right.$ par , $x \varepsilon \alpha^{\prime},, g x^{\prime}$ par ,$x \varepsilon \beta^{\prime}$, , hx' par,$x \varepsilon \gamma^{\prime}$ dans $Y$, dans laquelle chaque groupe de points a été substitué par un groupe plus grand d'une unité".

Exemple: $,(x): x \varepsilon a . \equiv . x \varepsilon \alpha^{\prime \prime}$ est une expression analogue (16.11) de ". (x) $\cdot f x \equiv f x^{\prime \prime}(12.53)$. 
16.12. Toute expression analogue (16.11) a une loi du $\S 12$ ou obtenue en vertu des règles du $\S 12.5$ est une loi.

16.2. Lois principales.

$$
\begin{aligned}
& \text { 16.211. } \alpha \cup \beta \cdot=\cdot \beta \cup \alpha \quad(5.22) \\
& \text { 16.212. } \alpha \cap \beta \cdot=\cdot \beta \cap \alpha \quad(5.52) \\
& \text { 16.221. } \alpha \cdot \cup \cdot \beta \cup \gamma:=: \alpha \cup \beta \cdot \cup \cdot \gamma \quad \text { (5.23) } \\
& \text { 16.222. } \alpha \cdot \cap \cdot \beta \cap \gamma:=: \alpha \cap \beta \cdot \cap \gamma \quad(5.53) \\
& \text { 16.231. } \alpha \cup \alpha \cdot=\alpha \quad(5.15) \\
& \text { 16.232. } \alpha \cap \alpha \cdot=\alpha \quad(5.16) \\
& \text { 16.241. } \alpha=\alpha \quad(5.11) \\
& \text { 16.242. } \alpha \subset \alpha \quad(5.11 \text { cf. 5.73) } \\
& \text { 16.243. }--\alpha=\alpha \quad(5.12) \\
& \text { 16.25. } \alpha \subset \beta \equiv-\beta \subset-\alpha \quad(5.32) \\
& \text { 16.26. } \alpha \subset \beta: \supset: \beta \subset \gamma \cdot \supset \cdot \alpha \subset \gamma \quad(6.32) \\
& \text { 16.27. } \alpha \subset \beta \cdot \equiv \cdot \alpha \cap \beta=\cdot \alpha \quad(5.314) \\
& \text { 16.28. } \alpha \subset \beta \cdot \equiv \cdot \alpha \cup \beta=\cdot \beta \quad \text { (5.315) } \\
& \text { 16.29. } \alpha \subset \beta \cdot x \varepsilon \alpha \cdot \supset x \varepsilon \beta \quad(12.91)
\end{aligned}
$$

16.3. Lois de la classe universelle et vide.

$$
\begin{aligned}
& \text { 16.311. } \wedge=-\vee \\
& \text { 16.312. } \wedge \neq \vee \\
& \text { 16.313. } \vee=-\wedge \\
& \text { 16.321. }(x) \cdot x \varepsilon \vee \\
& \text { 16.322. }(x) \cdot x \sim \varepsilon \wedge \\
& \text { 16.323. }(\alpha) \cdot \alpha \subset \vee \\
& \text { 16.324. } \wedge \subset \alpha \\
& \text { 16.331. }(x) \cdot x \varepsilon \alpha \cdot \equiv \cdot \alpha=\vee \\
& \text { 16.332. }(x) \cdot x \sim \varepsilon \alpha \cdot \equiv \cdot \alpha=\wedge \\
& \text { 16.341. } \alpha=\vee \cdot \equiv \cdot-\alpha=\wedge \\
& \text { 16.342. } \alpha \cup-\alpha=\vee \\
& \text { 16.343. } \alpha \cap-\alpha=\wedge \\
& \text { 16.344. } \alpha \cup \wedge=\alpha \\
& \text { 16.345. } \alpha \cap \wedge=\wedge \\
& \text { 16.346. } \alpha \cup \vee=\vee \\
& \text { 16.347. } \alpha \cap \vee=\alpha \\
& \text { 16.351. } \alpha \subset \beta . \equiv \cdot-\alpha \cup \beta \cdot=\cdot \vee \\
& \text { 16.352. } \alpha \subset \beta \cdot \equiv \cdot \alpha \cap-\beta \cdot=\cdot \wedge \\
& \text { 16.353. }-\alpha \subset \beta \cdot \equiv \cdot \alpha \cup \beta \cdot=\cdot \vee
\end{aligned}
$$


16.354. $\alpha \subset-\beta . \equiv \cdot \alpha \cap \beta \cdot=\cdot \wedge$

16.361. $\alpha \cup \beta \cdot=\cdot \wedge \cdot \equiv \cdot \alpha=\wedge \cdot \beta=\wedge$

16.362. $\alpha \cap \beta \cdot=\cdot \wedge \cdot \equiv \cdot \alpha=\wedge \cdot \vee \cdot \beta=\wedge$

16.363. $\alpha \cap \beta \cdot=\cdot \wedge \cdot \equiv \cdot \alpha \cdot=\cdot \alpha \cap-\beta$

16.364. $\alpha \cap \beta \cdot=\cdot \wedge: \equiv:(x, y): x \varepsilon \alpha \cdot y \varepsilon \beta \cdot \supset \cdot x \neq y$

16.371. $\alpha:=: \alpha \cap \beta \cdot \cup \cdot \alpha \cap \sim \beta$

16.372. $\beta \subset \alpha: \supset: \alpha:=\beta \cup \cdot \alpha \cap-\beta$

\subsection{Lois de l'existence.}

16.411. $\sim$ ヨ! $\alpha \cdot \equiv \cdot \alpha \cdot=\wedge$

16.412. $\exists ! \alpha \cdot \equiv \cdot \alpha \neq \wedge$

16.421. $\mathrm{B!} \mathrm{V}$

16.422. $\sim \exists ! \wedge$

16.431. $\exists !(\alpha \cup \beta): \equiv: \exists ! \alpha \cdot v \cdot \exists ! \beta$

16.432. $\exists !(\alpha \cap \beta): \equiv: \exists ! \alpha \cdot \exists ! \beta$

16.433. $\alpha \subset \beta: \supset: \exists ! \alpha \cdot \supset \cdot \exists ! \beta$

$L$ 'inverse n'est pas vrai.

16.44. $\sim \alpha \subset \beta: \equiv: \exists !(\alpha \cap-\beta)$

16.451. $\alpha \cap \beta \cdot=\cdot \wedge: \supset: \exists ! \alpha \cdot \supset \cdot \alpha \neq \wedge$

16.452. $\exists ! \alpha \cdot \alpha=\beta \cdot \supset$. $\exists !(\alpha \cap \beta)$

16.453. $\alpha \subset \beta \cdot \alpha \neq \beta \cdot \equiv \cdot \exists !(\alpha \cap-\beta)$

16.461. $\sim$ घ! $\beta \cdot \supset \cdot \alpha \cup \beta \cdot=\cdot \alpha$

16.462. $\sim \exists ! \beta \cdot \supset \cdot \alpha \cap \beta \cdot=\cdot \wedge$

HISTOIRE ET LITTÉRATURE: voir $§ 15$.

§ 17. ANTINOMIES ET THÉORIE Ce chapitre présente un exposé rudimenDES TYPES

taire et non-formalisé des antinomies (dites aussi „paradoxes") qui apparaissent dans les systèmes logiques, et des règles qui servent à les éliminér; un groupe de ces règles porte le nom de la ",théorie des types".

\subsection{Antinomies.}

17.11. „Antinomie" pour: ,produit logique d'une proposition et de son isomorphe niée, ou d'une expression équivalente".

Exemples: , $p . \sim p ", \ldots(x) f x .(E x) \sim f x "$ sont des antinomies.

17.12. Dans tout système logique suffisamment formalisé, dans 
lequel on n'observe pas des précautions spéciales, un nombre indéfini d'antinomies peut être déduit.

17.13. Les antinomies se divisent en antinomies métalogiques et antinomies logiques.

17.14. „Antinomie logique” pour „,antinomie qui résulte même si les suppositions sont clairement distinguées".

17.15. "Antinomie métalogique" pour ,antinomie qui résulte de la confusion des suppositions (2.1)".

Exemples: 17.15: antinomie du menteur (cf. 17.2); 17.14: antinomie de la classe des classes (17.7).

\subsection{Antinomie de la classe des classes.}

17.21. „Antinomie de la classe des classes (russellienne)" pour: ,,antinomie qui résulte de l'introduction dans un système des expressions du type , $\alpha \varepsilon \alpha^{\prime}$."

17.22. Dans chaque système logique formalisé qui contient les lois et les règles exposées aux $\S \S$ précédents l'introduction de , $\alpha \varepsilon \alpha$ " permet de déduire une antinomie.

Justification: si „a $\alpha a^{\prime \prime}$ est une expression, on peut définire (15.11) une classe $\beta$ telle que, pour tout $\alpha$

$$
\text { (1) } \alpha \varepsilon \beta . \equiv \cdot \sim, \alpha \varepsilon \alpha
$$

En substituant , $\beta$ " pour ,,$\alpha$ " on obtient

et de là

$$
\text { (2) } \beta \varepsilon \beta . \equiv \cdot \sim \beta \varepsilon \beta
$$

(3) $\beta \varepsilon \beta, \sim \beta \varepsilon \beta$

ce qui est une antinomie (17.11).

\subsection{L'expression „, $\alpha \varepsilon a^{\prime \prime}$ n'a pas de sens.}

Justification: si elle avait un sens, (1) serait vrai; or (1) n'est pas vrai; donc elle n'a pas de sens. Elle semble être une proposition, mais n'en est pas une. C'est un ensemble de signes qui ne signifient rien.

\section{7,3. Théorie des types.}

17.31. "Théorie des types" pour ,ensemble de règles qui, en divisant les objets ou les expressions de la logique en classes numérotées, (types) permet d'éviter les antinomies logiques".

17.32. "Théorie des types ontologiques" pour "théorie des types qui divise les objets en types". 
Explication: une théorie des types ontologiques a comme ler type l'ensemble des individus, comme $2 \mathrm{e}$ celui des classes des individus, comme $3 \mathrm{e}$ celui des classes des classes des individus etc.

17.33. „Théorie des types syntactiques" pour ,théorie des types qui divise les expressions en types."

Excmple: 17.4 .

\subsection{Règles des types syntactiques.}

17.41. „Types" pour: „,classes d'expressions mutuellement exclusives; autre nom des catégories sémantiques (1.22)".

Explication: toutes les expressions sont divisces en classes numérotés, dites "type 1", "type 2 " ... ,type $n$ ".

17.42. Règle: Toutes les expressions isomorphes du même système apartiennent au même type.

17.43. Règle: Si $F$ est l'opérateur de $X$ et $X$ apartient au type $n$, $F$ apartient au type $n+1$.

17.44. Règle: $\mathrm{Si} X$ est suivi de „, $\varepsilon$ " suivi de $Y$, et $X$ appartient au type $n, Y$ appartient au type $n+1$.

\subsection{Méthode de Quine.}

17.51. Pour vérifier si la règle 17.44 est appliquée, on procède comme suit: (a) Substituer toutes les variables isomorphes arbitrairement choisies par „O", (b) si une variable est suivie de „ $\varepsilon$ " suivi luimême d'un chiffre, substituer à cette variable un chiffre plus petit d'une unité (positif, ,.O" ou négatif), (c) répéter l'opération jusqu'au moment où toutes les variables seront substituées par des chiffres; au besoin recommencer avec une autre variable; (d) si alors le chiffre qui suit chaque ,,$"$ " est toujours d'une unité plus grand que celui qui le précède, l'expression est conforme à la règle des types; si non, elle ne J'est pas.

17.52. Pour vérifier si la règle 17.43 est appliquée, substituer

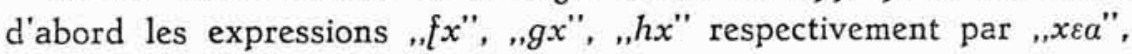
, $x \varepsilon \beta^{\prime \prime},,, x \varepsilon \gamma^{\prime \prime}$, ensuite appliquer 17.51.

\subsection{Principe d'analogie.}

17.61. L'application de la règle des types oblige à distinguer différents types d'opérateurs constants $, \varepsilon^{\prime \prime},,=",, \neq=$ etc. et des expressions , $v "$ et ", $\wedge$ ".

Explication: la règle des types s'applique aussi aux opérateurs dyadiques, tels que,$="$. Par conséquent, si $"="$ dans,$x=x^{\prime \prime}$ appartient au type $n$, et si 
d'autre part nous avons,$x \varepsilon \alpha^{\prime \prime}$, on ne peut pas, conformément à la règle des types, écrire,$\alpha=\alpha^{\prime \prime}$, car " $\alpha "$, étant d'un type supérieur à $x$, le , $="$ qui unit deux , $a$ " devra être aussi d'un supérieur au premier,$="$, ce qui est contraire à 17.42 .

17.62. Pour éviter la multiplication des expressions et des lois pour chaque type, la règle 17.42 n'est pas appliquée aux opérateurs énumérés en 17.61 .

17.63. Principe de l'analogie ou équivocité systématique. Les opérateurs , $, \varepsilon ",,=",, \ldots=$ etc. et les expressions ,,$"$ et , $\wedge "$ sont systématiquement équivoques quant à leur type.

Explication: Les expressions de cette forme ont un sens différent d'après le type, mais leurs propriétés formelles restent les mêmes. P. ex. lois 14.13-15 restent valides quand les noms (variables) des individus qu'elles contiennent sont remplacés par des noms de classes.

\subsection{Antinomie du menteur}

17.71. "Antinomie du menteur" pour: ,,antinomie qui résulte de l'introduction dans le système des expressions du type "X est faux".

17.72. Dans chaque système de logique formalisé qui contient des lois et les règles exposées aux chapitres précédents, l'introduction de „X est faux" permets de déduire l'antinomie du menteur, si des précautions spéciales ne sont pas observées.

Justification. On forme la proposition „c est faux" et prend „c" comme abbréviation typographique de cette proposition. On a donc:

(1) c est faux $=c$

D'autre part, d'après la définition courante de la vérité:

(2) $X$ est vrai. $\equiv . X$

En substituant , cc est faux" pour ", $X$ " dans (2) on obtient

(3) c est faux est vrai . 三. c est faux.

En substituant (en vertu de l'identité (1)) ,c" pour „c est faux" on obtient de (3):

(4) c est vrai. $\equiv$. c est faux

D'où, puisque $c$ est faux. $\equiv . \sim$. c est vrai, on obtient l'antinomie.

\subsection{Solution des antinomies métalogiques.}

17.81. Règle: Afin d'éviter les antinomies métalogiques il est nécessaire d'observer strictement les règles de la supposition (2.13 ou 2.14).

17.82. Si la règle 2.13 est appliquée, l'antinomie du menteur n'apparait pas.

Just:fication: dans ce cas, au lieu de (1) et (2) on obtient, respectivement 
(1') $c$ est faux $=c$

(2') , $X^{\prime \prime}$ est vrai $\equiv X$

mais l'on ne peut plus procéder ultérieurement, puisque " $X "$ au commencement de $\left(2^{\prime}\right)$ est le nom de la lettre ix et ne saurait être substitué.

Une solution pleinement satisfaisante de l'antinomie du menteur demande, cependant, une élaboration préalable de la définition de la vérité. Quand on ne dispose pas d'une telle définition, il faut toujours éviter de faire usage des mots „vrai" et "faux".

HiSTOIRE: En 1908 Russell et Zermelo donnèrent simultanément deux solutions différentes du problème. La théorie „simple” de Russell, reprise dans les $P M$, fut développée en une théorie "ramifiée" ( $P M$, le édition). Puis ( $P M, 2$ e édition) une tendance à la simplifier se manifesta qui semble croître; certains, comme Ushenko, pensent même qu'on pourrait se passer d'une théorie générale des types. - La distinction entre les paradoxes logiques et métalogiques est de Ramsay.

LitTÉRATURE: Russell, Introduction; PM (Intr. à la 2e éd.); Zermelo, Untersuchungen; Chwistek, Antynomie; Ramsey, The Foundations; Tarski, Wahrheitsbegriff; Quine, Types; Math. Logic; Church, A formulation; Fraenkel et BarHillel, Le problème. Systèmes sans th. des types: Fitch, A system; Ackermann, Ein System. - Discussion: Ushenko, Problems. - Histoire: Rüstow, Der Lügner; Salamucha, Pojawienie.

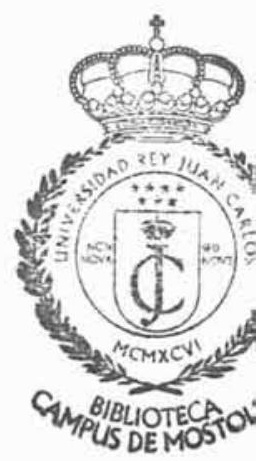


RELATIONS

§ 18. RELATIONS La théorie des relations correspond à la théorie des prédicats dyadiques $(\S 13)$, comme celle des classes $(\S 15)$ à la théorie des prédicats monadiques $(\S 11)$. C'est la partie la plus neuve et la plus importante de la logique moderne. Elle fut développée pour fonder les mathématiques, mais son champ d'application dépasse cette science et embrasse l'ensemble du savoir. Malgré qu'elle occupe la majeure partie des traités actuels, elle est encore relativement bien peu développée.

\subsection{Définitions.}

18.11. ", $\hat{x} \hat{y}\{f(x, y)\} "$ pour: ,"les $x$ et les $y$ tels que $f(x, y)$ ".

Explication: cf. 15.11; les couples ainsi définis sont nommés „relations”. La "relation" est donc prise en extension.

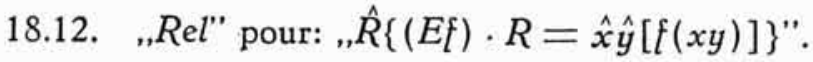

Explication: cf. 15.12. Rel est la classe des relations, c. à d. des couples définis en 18.11 .

18.13.,$u\{\hat{x} \hat{y}[f(x, y)]\} v^{\prime \prime}$ pour: , $\{(u, v) "$.

18.14. "uRv" pour: , $u\{\hat{x} \hat{y}[f(x, y)]\} v^{\prime \prime}$.

Explication: $18.13-14$ servent à introduire la nouvelle écriture „xRy".

18.15. „Antécédent de $R$ " pour: ,objet qui a la relation $R$ à quelque chose".

18.16. „Conséquent de $R$ " pour: ,,objet auquel quelque chose a la relation $R^{\prime \prime}$.

18.17. "Terme de $R$ " pour: ,,antécédent ou conséquent de $R$ ".

\subsection{Relations entre relations.}

18.21. "亡R" pour , $\hat{x} \hat{y}(\sim x R y) "$.

Explication: $\doteq R$ est la relation complémentaire de $R$, (cf. 15.21) c. à d. la classe de tous les couples non-liés par la relation $R$. Exemple: la relation complémentaire de "frère" est l'ensemble des couples qui sont amis, voisins, plus grands que, supérieurs de, semblables à, etc. mais qui ne sont pas des frères. 
18.22. "RUं $S$ " pour" , , $\hat{x} \hat{y}(x R y \vee x S y)$ "

18.23. " $R \dot{\mathrm{n}} S$ " pour: , , $\hat{x} \hat{y}(x R y \cdot x S y) "$

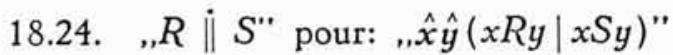

18.25. , $R \subset \cdot S$ " pour: , $(x, y) \cdot x R y \supset x S y "$

18.26.,$R \doteq S "$ pour: ,. $(x, y) \cdot x R y \equiv x S y "$

18.27. " $\ddot{\mathrm{v}}$ " pour: , $\hat{x} \hat{y}(x=x \cdot y=y) "$

18.28. ,,$\dot{\wedge}$ " pour: , $\hat{x} \hat{y}(x \neq x \cdot y \neq y)$

18.29. , ,安!R" pour: „(Exy)xRy"

On emploi les mêmes noms que pour les classes: „somme de relations”, „relation vide" etc.

\subsection{Lois analogues.}

18.31. ,X est une expression analogue (18.3) à $Y$ " pour: , $X$ est une expression formée par la substitution de

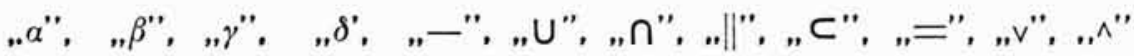
respectivement par:

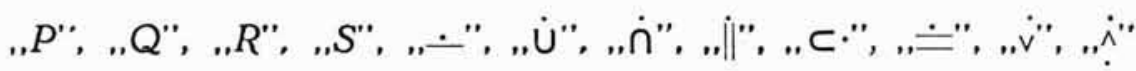
dans $Y$.

18.32. Toute expression analogue (18.3) à une loi du calcul des classes ( $\$ 16$, les lois formées en vertu de $16.11 \mathrm{~s}$. inclues) est une loi.

HISTOIRE: L'idée de définir la relation comme classe de couples est de Peirce; elle fut reprise et considérablement développée par Frege et Peano. La forme actuelle est due aux $P M$. Récemment, Wiener et Kuratowski fondèrent la théorie sur une nouvelle base.

LitTÉRATURE: PM ${ }^{\star} 21,{ }^{\star} 23,{ }^{\star} 25$ et les autres traités; pour les développements nouveaux: Wiener, Simplification; Kuratowski: Notion d'ordre; Tarski, On the Calculus; Quine, Math. Logic § 36 . Un exposé élémentaire très clair chez Carnap Abriss, reproduit par Boll, Logique.

$\S$ 19. Descriptions Relatives; Converse

Les descriptions ont une importance particulière quand il s'agit de prédicats dyadiques (relations): les expressions les plus courantes p. ex. en théologie, en droit et en mathématiques sont, en effet, des descriptions relatives. Onc connait plusieurs genres différents de ces descriptions. 
- A la fin du paragraphe sera donnée une autre théorie importante, celle de la converse d'une relation.

\subsection{Descriptions individuelles et plurielles.}

19.11. "R' $y$ " pour: ,, $(1 x)(x R y) "$.

Explication: cette expression porte le nom de „,description relative individuelle", car elle décrit un seul individu qui a une relation donnée à un seul autre (cf. 14.22). Exemple: si "R" signifie ,auteur de" et , $a$ ", "l'Iliade”, "R' $a$ " signifiera: "l'auteur de l'Iliade". Cette expression serait privée de sens si la théorie de Wolff était vraie, c. àd. si l'Iliade avait plusieurs auteurs.

19.12. " $\vec{R}^{\prime} y$ " pour: ,, $\hat{x}(x R y) "$

19.13. " $\stackrel{\leftarrow}{R^{\prime}} x "$ pour: ,. $\hat{y}(x R y) "$

Explication: ces deux expressions sont dites „descriptions relatives plurielles”, car elles signifient la classe des objets qui ont la relation $R$ à un individu donné $\left(, \vec{R}^{\prime} y^{\prime \prime}\right)$ ou auquel un individu donné a la relation $R\left(\stackrel{\leftarrow R^{\prime}}{x^{\prime \prime}}\right)$.

Exemple: si „R" signifie , ,auteur de”, et „a“, ,la Bible”, , $\overrightarrow{R^{\prime}} a^{\prime \prime}$ sera ,la classe des auteurs de la Bible”; si „, $a$ " signifie „Homère”, „ $\leftarrow R^{\prime} a "$ signifiera: „les oeuvres (la classe des oeuvres) d'Homère".
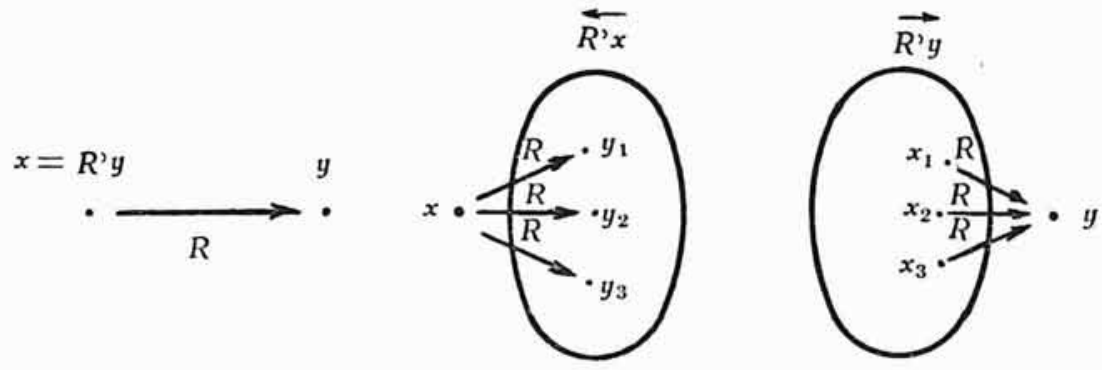

19.14. ",sg'R" pour: , $\vec{R}$ ".

19.15. .,gs' $R$ " pour: , $\stackrel{\leftarrow}{R .}$

Explication: ,sg' $R$ " (de: „sagitta”) et „gs' $R$ ” (de „sagitta" inverse) servent à remplacer,$\vec{R}^{\prime \prime}$ et,$\stackrel{\leftarrow}{R^{\prime \prime}}$ quand il s'agit de formules plus longues, p. ex. de sommes, produits de relations etc.

\subsection{Description bi-plurielle.}

19.21. ",R' ' $\beta$ " pour: , , $\hat{x}\left\{\left\{\left(E_{y}\right) \cdot y \varepsilon \beta \cdot x R y\right\} "\right.$. 
Explication: $R$ ' $\beta$ est la classe des individus aui ont la relation $R$ à un élément quelconque de la classe $\beta$.

Exemple: si "R" est „auteur de" et $\beta$ est la classe des écrits en vers, $R$ ' ' $\beta$ sera la classe des auteurs des écrits en vers ou, comme plusieurs le pensent (à tort), des poètes.

Représentation graphique:

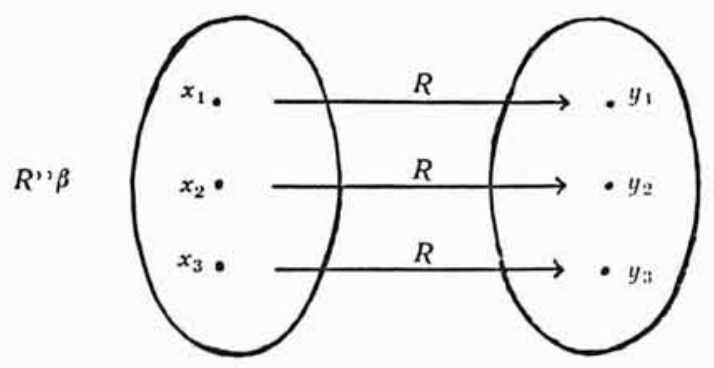

19.22. $(\alpha, \beta, R): \alpha \subset \beta . \supset \cdot R{ }^{\prime \prime} \alpha \subset R \prime \prime \beta$

Exemple: si les chevaux sont des animaux, les têtes des chevaux sont des têtes d'animaux.

\subsection{Converse.}

19.31. , , $x \breve{R} y "$ pour: , $y R x "$.

Exemple: si „R" est ,l'auteur de", , $\breve{R " ~ s e r a ~ „ l ' o e u v r e ~ d e " . ~} \mathrm{Si}$ „R" est „à droite de", , $\breve{R} "$ sera , à gauche de".

19.32. „Cnv'R" pour: „ц̌R”.

Explication: Cnv est une relation qui tient entre $R$ et $\breve{R}$; la description "Cnv'R" sert à remplacer " $\breve{R} "$ quand „R" est substitué par une expression plus longue. Représentation graphique:

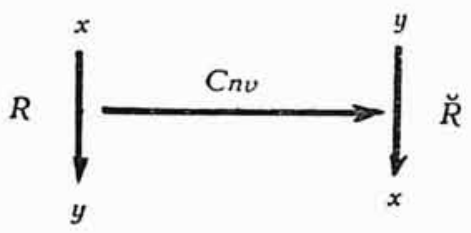

19.4. Lois de la converse.

19.41. $R \doteq S . \equiv . \breve{R} \doteq \breve{S}$

19.42. $C n v^{\prime} C n v^{\prime} R \doteq R$

19.43. E!Cnv'R 
19.44. $C n v^{\prime}(R \cup \dot{U} S) \cdot C^{\prime} n v^{\prime} R \cup \dot{U} C n v^{\prime} S$

19.45. $C n v^{\prime}(R \cap \dot{S}) \cdot=\cdot C n v^{\prime} R \cap \dot{C} n v^{\prime} S$

19.46. $C n v^{\prime} \doteq R \doteq \doteq C n v^{\prime} R$

19.47. $R \doteq \breve{S} . \equiv \cdot S \doteq \breve{R}$

19.48. $R \subset \cdot \breve{S} . \equiv . S \subset \cdot \breve{R}$

Historre: La théorie des descriptions relatives fut déjà traitée par de Morgan, qui donna l'exemple 19.22; celle de l'inverse fut élaborée par les mathématiciens adonnés à la théorie des ensembles. Elle fut déjà examinée par Cayley en 1854 . Frege et Peano, fondateurs de la logique contemporaine, et les auteurs des PM lui donnèrent sa forme actuelle.

LitTérature: PM *30- 32 et tous les traités.

\$20. DOMAINES ET Champs; UNIVOCITÉ RELATIVE

En plus des expressions exposées au $§ 19$. il $\mathrm{y}$ a encore des notions semblables, mais plus générales: en effet, on emploie souvent non seulement des expressions du type ,la mère de", "le voisin de", mais aussi du type plus général: „les mères", „les voisins". La théorie de ces expressions est suivie dans ce chapitre de quelques informations sur les relations limitées d'un côté ou de deux à une classe unitaire.

\subsection{Domaines et champs.}

20.11. "D'R" pour: , $\hat{x}\{(E y) x R y\} "$.

Explication: $C^{\prime} R$ est le champ de $R$, c. à d. la somme logique du domaine et du codomaine de $R$.

20.12. " „G'R" pour: , $\hat{y}\{(E x) x R y\}^{\prime \prime}$.

Explication: $Q^{\prime} R$ est le co-domaine de $R$, c. à d. l'ensemble des objets auxquels d'autres objets quelconques ont la relation $R$.

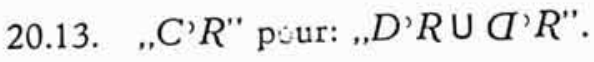

Explication: $C^{\prime} R$ est le champ de $R$, c. à d. la somme logique du domaine et du codomaine de $R$.

Exemples: si , $R$ " signifie "supérieur militaire de", „D' $R "$ signifie la classe de tous ceux qui sont des supérieurs militaires, c. à d. de tous les officiers et sous-officiers (de toutes les armées); " $Q^{\prime} R$ " la classe de tous ceux qui ont des supérieurs militaires, c. à d. de tous les soldats, sauf les commandants en chef; enfin "C' $R$ " la classe des uns et des autres. $\mathrm{Si}, R^{\prime \prime}$ signifie „parent de", "D' $D^{\prime \prime}$ signifiera la classe de tous les hommes et femmes qui ont des enfants, ", $Q^{\prime} R^{\prime \prime}$ celle de tous ceux qui ont des parents (c. à d. de tous les êtres humains exceptés Adam et Eve).

La différence entre $D^{\prime} R$ et $\vec{R}^{\prime} y$ comme aussi entre $D^{\prime} R$ et $R^{\prime \prime} \alpha$ consiste en 
ceci que $\vec{R}^{\prime} y$ est la classe des objets qui ont la relation $R$ à un individu déterminé $y$, et $R$ ' ' $\alpha$ celle des objets qui ont cette relation aux éléments d'une classe déterminée $\alpha-$ tandis que $D^{\prime} R$ est la classe de tous les objets ayant la relation $R$ à un objet quelconque.

\subsection{Lois des domaines et des champs.}

20.21. $(x, y): x R y \cdot \supset \cdot x \varepsilon D^{\prime} R \cdot y \varepsilon Q^{\prime} R$

20.22. $\quad(y) \cdot \vec{R}^{\prime} y \subset D^{\prime} R$

20.23. $(x) \cdot \stackrel{\leftarrow}{R^{\prime}} x \subset Q^{\prime} R$

20.24. $D^{\prime} R=Q^{\prime} \ddot{R}$

20.25. $C^{\prime} R=C^{\prime} \not{R}$

20.26. $C^{\prime} R=C^{\prime}(R \dot{U} \check{R})$

20.27. $D^{\prime} R \subset Q^{\prime} R \cdot \equiv \cdot G^{\prime} R=C^{\prime} R$

Explication: si le domaine de $R$ est inclus dans le co-domaine de $R$, ce dernier est égal au champ de $R$. Dans ce cas la série formée par $R$ n'a pas de commencement; car quel que soit le terme de $R$, il est toujours un élément de $Q^{\prime} R$, c. à d. il a un antécédent.

\subsection{8. $Q^{\prime} R \subset D^{\prime} R \cdot \equiv \cdot D^{\prime} R=C^{\prime} R$}

Explication: la série n'a pas de fin ici, car, quel que soit le terme de $R$, il est toujours un élément de $D^{\prime} R$, c. à d. il a un conséquent.

\subsection{Relations aux domaines limités.}

20.31. ", $\alpha 1 R$ " pour: , , $\hat{x} \hat{y}(x \varepsilon \alpha \cdot x R y) "$

20.32. "R+ $\beta$ " pour: , $\hat{x} \hat{y}(y \varepsilon \beta \cdot x R y) "$

20.33. " $\alpha \uparrow R+\beta "$ pour: , $\hat{x} \hat{y}(x \varepsilon \alpha \cdot y \varepsilon \beta \cdot x R y) "$

20.34. " $R \uparrow \alpha "$ pour: , $\alpha \uparrow R \vdash \alpha "$

Explication: $20.31-34$ introduisent la notion de relation à domaines et champs limités. Ainsi $\alpha_{1} R$ est la relation $R$ limitée dans son domaine à la classe $\alpha, R \uparrow \beta$ la même relation limitée dans son co-domaine à la classe $\beta, \alpha \uparrow R+\beta$ la relation $R$ limitée dans son domaine à la classe $\alpha$ et dans son co-domaine à la classe $\beta$; enfin $R\lceil\alpha$ est la relation $R$ dont le champ est limité à la classe $\alpha$.

Exemple: si "R" est ,auteur de" et " $\alpha$ " est ,italien”, $\alpha \uparrow R$ sera la relation d'auteur limitée dans son domaine aux italiens; dans ce cas, $D^{\prime}\left(\alpha_{1} R\right)$ sera la classe des auteurs italiens et $Q^{\prime}(R \uparrow a)$ celles des oeuvres italiennes. 
20.35. , $\alpha \uparrow \beta "$ pour: , , $\hat{x} \hat{y}(x \varepsilon \alpha \cdot y \varepsilon \beta)$

20.36. $\alpha \uparrow \beta=\alpha 1 \dot{v} \uparrow \beta$

Explication: $\alpha \uparrow \beta$ est la relation qui existe entre $x$ et $y$ par le fait même que $x$ est un élément de $\alpha$ et $y$ un élement de $\beta$; c'est donc le sens qu'obtient „ $\alpha 1 R \uparrow \beta$. si " $R$ " est substitué par , , ${ }^{\prime \prime}$. Cette notion joue un rôle considérable dans la théorie des séries.

20.37. , ,x $\downarrow \downarrow y$ " pour: , , $[x] \uparrow[y]^{\prime \prime}$

Explication: 20.37 donne la définition du couple ordinal.

\subsection{Univocité relative.}

20.41. " $1 \rightarrow$ cls" pour: , $\hat{R}\{(x, y, z): x R z \cdot y R z \cdot \supset \cdot x=y\} "$

20.42. „cls $\rightarrow 1$ " pour:, , $\{((x, y, z): x R y \cdot x R z \cdot \supset \cdot y=z\} "$

20.43. , $1 \rightarrow 1$ " pour: , $(1 \rightarrow c l s) \cap(c l s \rightarrow 1) "$

Explication: 20.41 définit la relation univoque, c. à d. limitée dans son domaine aux classes unitaires; 20.42 , la relation réciproque, limitée de la même manière dans son co-domaine; enfin 20.43 définit la relation bi-univoque dont le domaine et le co-domaine sont limités à des classes unitaires. 20.41 dit, en effet, que chaque fois que $x R z$ et $y R z$, il $\mathrm{y}$ a identité entre $x$ et $y$, donc qu'il ne peut $\mathrm{y}$ avoir qu'un seul antécédent de $R$ - et 20.42 dit le même du conséquent.

Exemples: la relation „père" est univoque, car le même père peut avoir plusieurs enfants, tandis qu'un enfant ne peut avoir qu'un seul père. La relation "mari de" serait univoque et la relation "femme de" réciproque, selon le Coran; mais pour les chrétiens, les deux relations sont bi-univoques.

20.44. $\operatorname{Cnv}^{\prime}(1 \rightarrow c l s)=(c l s \rightarrow 1)$

LitTérature: $\S 20.1-2: \mathrm{PM} * 33 ; \S 20.3: \mathrm{PM}$ *35; $20.4: \mathrm{PM}$ *71; Carnap, Abriss.

§ 21. ENCHAINEMENT RELATIF; L'enchainement relatif est une des notions SÉRIES les plus importantes pour toutes les sciences qui, comme les mathẻmatiques et la théologie, utilisent le concept de série. Le présent chapitre en donne les notions fondamentales et quelques applications élémentaires à la doctrine des séries. Cette doctrine forme, à elle seule, un traité très étendu.

\subsection{Produit relatif.}

21.11. , $R \mid S$ " pour: , $\hat{x} \hat{z}\left\{\left(E_{y}\right) \cdot x R y \cdot y S z\right\}$ "

Explication: $R \mid S$ est le produit relatif de $R$ et de $S$, c. à dire la relation qui existe entre $x$ et $z$ s'il existe un $y$ tel que $x R y$ et $y R z$. 
Exemples: le produit relatif de frère et de père est le frère du père, $\mathrm{c}$. à $\mathrm{d}$. l'oncle; le produit relatif de carré et de moitié est le carré de la moitié.

21.12. " $R^{2}$ " pour: , $R \mid R$ "

21.13. ",R ${ }^{3 "}$ pour: , $R^{2} \mid R^{\prime \prime}$

21.14. " $R^{n "}$ pour: ", $R^{n-1} \mid R^{\prime \prime}$

21.15. " $R^{\circ}$ " pour: , $I+C ' R "$

Explication: les expressions $21.12-15$ portent le nom de „puissances relatives" (,carré relatif", ,cube relatif" etc.). $R^{\circ}$ est l'identité (cf. 14.16) limitée au champ de $R$, c. à d. la relation d'identité que chaque élément de $C^{\prime} R$ a à soi-même; cette notion joue dans les séries un rôle analogue à celui du zéro en mathématiques.

Exemples: si "R" signifie „père de", "R $R^{2}$ " signifie „grand père paternel de". L'adage "les amis de mes amis sont mes amis" se traduit par " $R^{2} \subset R$, , هi " $R$ " signifie „ami de".

\subsection{Relation ancestrale.}

\subsection{1. "her" pour: , $\hat{a}\left\{(\exists \breve{R})\left(R^{\prime \prime} \alpha \subset \alpha\right)\right\}$ "}

Explication: une classe est dite ,héréditaire" à l'égard de la relation $R$ (her est la classe des classes héréditaires), quand les conséquents de $R$ par rapport aux éléments de $\alpha$ sont des éléments de $\alpha$.

Exemple: la classe des Hongrois est héréditaire à l'égard de la relation de père, car si $x$ est le père de $y$ et $x$ appartient à la classe des Hongrois, c. à $\mathrm{d}$. s'il est un Hongrois, $y$ est aussi un Hongrois.

21.22. ",R. " pour: ,, $\hat{x} \hat{y}\left\{x \varepsilon C^{\prime} R: \cdot(\alpha): \check{R}{ }^{\prime}{ }^{\prime} \alpha \subset \alpha \cdot x \varepsilon \alpha \cdot \supset \cdot y \varepsilon \alpha\right\} "$

Explication: 21.22 est une définition ingénieuse de la notion vague ", $R^{\circ} \cup \dot{U} R^{2} \cup \dot{ } R^{3}$ etc.", donc de la relation qui existe là où est donnée une puissance quelconque de $R$. Cette relation est dite „relation ancestrale".

Exemples: si "R" signifie „père de", „R " signifie „ancêtre paternel”; si „R" signifie „situé immédiatement à gauche en ligne droite de", „R * signifie "situé à gauche (à distance quelconque) en ligne droite de"; si " $R$ " signifie "supérieur immédiat", "R." signifie „supérieur (immédiat ou quelconque)".

21.23. ",R po" pour: $\hat{x} \hat{y}\left\{(\alpha): \breve{R^{\prime}{ }^{\prime}} \alpha \subset \alpha \cdot \stackrel{\leftarrow}{R}{ }^{\prime} x \varepsilon \alpha \cdot \supset \cdot y \varepsilon \alpha\right\} "$

Explication: $R_{p o}$ se distingue de $R_{*}$ par le fait qu'il exclut $R^{\circ}$. C'est donc l'équivalent de „RUं $R^{2} \dot{U} R^{3}$ etc.".

\subsection{Premiers et derniers termes.}

21.31. ",B" pour: ,, $\hat{x} \hat{R}\left\{x \varepsilon \cdot D^{\prime} R \cap-G^{\prime} R\right\}^{\prime}$ 
Explication: "B" (du mot anglais „beginning", „commencement") est la relation entre le premier terme $x$ de la série formée par $R$ et $R$ : en effet 21.31 dit que $x$ appartient au domaine mais pas au co-domaine de $R$. La classe des premiers termes de $R$ sera donc $s g^{\prime} B^{\prime} R$, celle des derniers $s g^{\prime} B^{\prime} C n v^{\prime} R$.

21.32.

$$
\text { "Min } \left.{ }_{R} \text { " pour: , } \hat{x} \hat{a}\left\{x \varepsilon \cdot a \cap C ' R \cap-\breve{R}{ }^{\prime}\right) \alpha\right\} \text { " }
$$

\subsection{3. ", $\operatorname{Max}_{R}$ " pour: "Min $\operatorname{Min}_{\breve{R}}$}

Explication: $\operatorname{Min}_{R}$ est $B$ mais limité à une classe; c'est le minimum de cette classe par rapport à $R$. $\operatorname{Max}_{R}$ est le maximum.

\subsection{Isomorphie des relations.}

\subsection{1. ",R†S" pour: , $R|S| \breve{R "}$}

Explication: la relation $R+S$ existe entre $x$ et $t$ quand on a $(\exists y, z) x R y, y S z$. $z \breve{R} t$ c. à d.

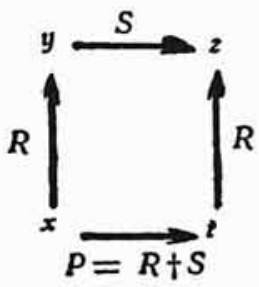

$R \dagger S$ est $l$ image de $S$ sur la base de $R$.

21.42. "Psmor $S^{\prime \prime}$ pour: ",रि $\left\{R \varepsilon 1 \rightarrow 1 \cdot C^{\prime} S=Q^{\prime} R \cdot P=R+S\right\}^{\prime \prime}$

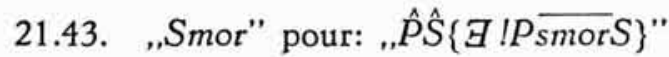

Explication: la relation $P$ est dite ,isomorphe" (, ,smor“, du latin „similis ordine") à $S$, quand il existe au moins une relation univoque $R$ telle que $P=R \dagger S$. (Ne pas confondre l'isomorphie des relations avec celle des termes cf. 1.15).

Exemple: La relation qui existe entre les pères de deux camarades d'école est isomorphe à celle qui existe entre ces garçons, si ils sont des fils uniques.

LITTÉRATURE: § 21.1: PM *34; § 21.2: PM *90, *91; § 21.3: PM *93; 21.4 : $\mathrm{PM}{ }^{*} 150,{ }^{\star} 151$. Carnap, Abriss.

\section{§22. PROPRIÉTÉS DES RELATIONS}

Dans ce chapitre on donne les définitions élẻmentaires de certaines propriétés communes à des grands groupes de relations telles que la réflexivité, transitivité, connexité etc. Cette théorie à une grande importance en logique supérieure et dans les mathématiques, tout en étant capable d'applications nombreuses dans d'autres domaines. 


\subsection{Réflexivité.}

22.11. "refl" pour: ", $\hat{R}\left(R^{\circ} \subset \cdot R\right)$ "

22.12. "irr" pour: ", $\hat{R}\left(R^{\circ} \subset \cdot \dot{-}\right)$ )"

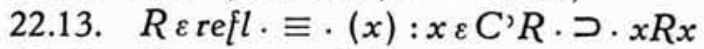

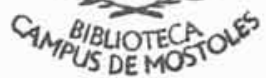

22.14. RE itr. $\equiv .(x) \sim x R x$

Explication: refl est la classe des relations réflexives c. à d. telles que si un $x$ appartient à leurs champs, ces relations tiennent entre $x$ et $x$. Par contre irt est la classe des relations irréflexives.

Exemples: l'identité et l'amour (selon Aristote) sont des relations réflexives, car tout être est identique à soi même et s'aime soi même (d'après ce philosophe). Par contre les relations d'être le père de, plus grand que, voisin de etc. sont irréflexives. - A noter qu'il y a des relation qui ne sont ni réflexives ni irréflexives, p. ex. celle de cuire la nourriture pour.

\subsection{Symétrie.}

22.21. "sym" pour: ", $\hat{R}(\breve{R} \doteq R)$ "

22.22. ",as" pour: ",R( $(\breve{R} \doteq-R)$ ”

22.23. $R$ ह sym. $\equiv \cdot(x) \cdot x R y \equiv y R x$

22.24. $R \varepsilon$ as. $\equiv \cdot(x) \cdot x R y \equiv \sim y R x$

Explication: sym est la classe des relations symétriques, as celle des relations asymétriques. Comme pour la réflexivité, il y a des relations qui ne sont ni symétriques, ni asymétriques.

Exemples: la relation d'être collègue de est symétrique, comme celle d'être voisin de; mais les relations d'être plus grand que, plus petit que, père de, fille de etc. sont asymétriques.

\subsection{Transitivité.}

22.31. "trans" pour: „, $\hat{R}\left(R^{2} \subset \cdot R\right)$ "

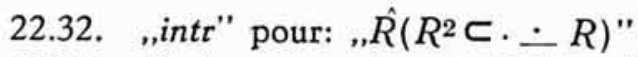

22.33. Retrans: $\equiv:(x, y):(E z) \cdot x R z \cdot z R y \cdot \supset \cdot x R z$

22.34. Re intr: $\equiv:(x, y, z): x R y \cdot y R z \cdot \supset . \sim x R z$

Explication: trans est la classe des relations transitives c. à d. celles qui .passent" d'un terme à l'autre; intr est la classe des relations intransitives. Même remarque que pour les relations réflexives et symétriques.

Exemples: la relation d'être à droite en ligne droite de, plus grand que, plus petit que, égal à, identique à, $\supset, \equiv,=, c$, est transitive; la relation d'être père de, fils de, mari de, femme de, carré de, est intransitive.

22.35. trans $\cap \mathrm{sym} . \subset$. $r e f l$

22.36. as $\subset$ irt 


\subsection{Similitude.}

\subsection{1. ",sim" pour: „sym $\cap$ refl”}

Explication: $\operatorname{sim}$ (du latin „similis") est la classe des relations de presqueidentité, comme „presque égal”, „de presque de la même couleur", etc. Toutes, elles ont les propriétés de symétrie et de réflexivité.

\subsection{2. „,eeq" pour: „trans $\cap$ sym".}

Explication: aeq (de ,aequalis") est la classe des relations d'égalité (de la même forme, couleur, grandeur etc. que...); ces relations sont toutes transitives et symétriques.

\subsection{3. aeq $\subset$ refl}

22.44. aeq $\subset \operatorname{sim}$

\subsection{Connexité.}

22.51. „,connex" pour: „,R $\left(J+C^{\prime} R \cdot \subset \cdot R \dot{U} \check{R}\right)$ ”.

Explication: une relation $R$ est dite „connexe” quand, entre deux objets différents quelconques qui appartiennent au champ de cette relation, existe toujours la relation $R$ ou $\breve{R}$.

\subsection{2. „set" pour: ,itr $\cap$ trans $\cap$ connex”.}

Explication: une relation est dite ,sériale", quand elle est irréflexive, transitive et connexe. C'est une relation de la plus haute importance pour les mathématiques et les autres sciences.

\subsection{3. ser $\subset$ irt}

\subsection{4. ser $\subset$ as}

22.55. Cnv'" ser $\subset$ ser

Littérature: PM *201, *202, *204; Carnap, Abriss.

$\S$ 23. RELATIONS à PLUS La théorie des relations binaires (à deux termes), DE DEUX TERMES quoique très importante, ne suffit nullement même dans les analyses les plus élémentaires des sciences non-mathématiques. Malheureusement, elle est la seule partie de la logique des relations qui fut élaborée. Nous donnons ici quelques définitions fondamentales d'une théorie généralisée.

\subsection{Définitions fondamentales.}

Dans les définitions qui suivent,$n$ " est une variable à substituer par des nombres entiers positifs. 
23.11. ", $\hat{x}_{1}, \ldots \hat{x}_{n}\left\{f\left(x_{1}, \ldots x_{n}\right)\right\} "$ pour ,les $\hat{x}_{1}, \ldots \hat{x}_{n}$ tels que $f\left(x_{1}, \ldots x_{n}\right) "$. Cf. 18.11 .

23.12. "Rel" pour ,,$\hat{R}\left\{(E f) \cdot R=\hat{x}_{1}, \ldots \hat{x}_{n}\left[\left\{\left(x_{1}, \ldots x_{n}\right]\right\} . " \mathrm{Cf}\right.\right.$. 18.12 .

23.13. ", $x_{1} R\left(x_{2}, \ldots x_{n}\right)$ " pour , $R\left(x_{1}, \ldots x_{n}\right) "$.

23.14. „Terme de $R$ " pour ,objet entrant d'une manière quelconque en relation $R$ avec des autres termes".

Explication: si $R$ a plus de 2 termes, on ne peut plus parler d'antécédents ni de conséquents de $R$ (cf. 18.15-16), mais seulement du n-tième terme de $R$. Le même est vrai pour le codomaine etc.

23.15. Les relations entre les relations à plus de 2 termes sont analogues à celles entre les relations binaires (cf. 18.2).

Exemple: pour une relation ternaire $R$ nous avons " $-R^{\prime \prime}$ pour

$$
\text { , } \hat{x}_{1}, \hat{x}_{2}, \hat{x}_{3}\left\{\sim R\left(x_{1}, x_{2}, x_{3}\right)\right\} " ;
$$

pour deux relations ternaires $R$ et $S$ nous aurons "RU் $S$ " pour

$$
\text { , } \hat{x}_{1}, \hat{x}_{2}, \hat{x}_{3}\left\{R\left(x_{1}, x_{2}, x_{3}\right) \vee S\left(x_{1}, x_{2}, x_{3}\right)\right\} " .
$$

Le sens des opérateurs est ici, évidemment, différent de celui qui fut définit au $\S 18.2$, mais le principe de l'analogie (17.6) peut être appliqué sans inconvénients.

23.16. La règle 18.32. s'applique aux relations à plus de 2 termes.

\subsection{Descriptions relatives.}

23.21. „, $R_{1}{ }^{\prime}\left(x_{2}, \ldots x_{n}\right) "$ pour ,.( $\left(x_{1}\right)\left(R\left(x_{1}, \ldots x_{n}\right)\right) "$. Cf. 19.11 .

23.22. ",Rk' $\left(x_{1}, \ldots x_{k-1}, x_{k+1}, \ldots x_{n}\right)$ " pour ,,( $\left(1 x_{k}\right)\left(R\left(x_{1} \ldots x_{k} \ldots\right.\right.$ (... $\left.\left.x_{n}\right)\right)$.

23.23.,$\vec{R}_{1}^{\prime}\left(x_{2}, \ldots x_{n}\right)^{\prime \prime}$ pour , , $\hat{x}_{1}\left\{R\left(x_{1}, \ldots x_{n}\right)\right\}^{\prime \prime}$. Cf. 19.12 .

23.24.,$\vec{R}_{k}{ }^{\prime}\left(x_{1}, \ldots x_{k-1}, x_{k+1}, \ldots x_{n}\right)^{\prime \prime}$ pour,$\hat{x}_{k}\left\{R\left(x_{1}, \ldots x_{k}, \ldots\right.\right.$ ... $\left.\left.x_{n}\right)\right\} "$.

23.25. "sgk' $R$ " pour , $\vec{R}_{k} "$. CF. 19.14 .

La théorie des descriptions multiplurielles (qui correspondent aux descriptions bi-plurielles, 19.2) est fort compliquée. Un cas limite simple est:

23.26. „R' $\left(\alpha_{2}, \ldots \alpha_{n}\right) "$ pour,$\left.\hat{x}_{1}\right\}\left(E x_{2}, \ldots x_{n}\right) \cdot x_{2} \varepsilon \alpha \cdot x_{3} \varepsilon \alpha_{3} \ldots$ $\left.\ldots x_{n} \varepsilon a_{n} \cdot R\left(x_{1}, \ldots x_{n}\right)\right\}$. Cf. 19.21 .

\subsection{Converses.}


23.31. Chaque relation à $n$ termes a $n !-1$ converses.

Explication: $n !=1.2 \ldots n$; ainsi pour $n=3, n !=1 \cdot 2.3=6$; une relation ternaire a donc $6-1=5$ converses, notamment celles qui tiennent entre les arguments suivants: (1) $1,3,2$, (2) $2,1,3$, (3) $2,3,1$, (4) $3,1,2$, (5) $3,2,1$.

23.32. " $R^{(a \ldots k \ldots u) " ~ o u ̀ ~, ~, a, ~} k, u$ " sont des variables à substituer par des nombres compris entre 1 et $n$, pour $, \hat{x}_{a}, \ldots \hat{x}_{k}, \ldots \hat{x}_{u}^{\prime}\left\{R\left(x_{1}, \ldots\right.\right.$ $\left.\left.\ldots x_{n}\right)\right\}^{\prime \prime}$.

Exemple: ",R(231)" pour $, \hat{x}_{2}, \hat{x}_{3}, \hat{x}_{1}\left\{R\left(x_{1}, x_{2}, x_{3}\right)\right\}$.

23.4. Domaines et champs.

23.41. "D, $D_{1}^{\prime} R$ " pour , $\hat{x}_{1}\left\{\left(E x_{2}, \ldots x_{n}\right) R\left(x_{1}, \ldots x_{n}\right)\right\} "$. Cf. 20.11.

23.42. "D, $D_{k}^{\prime} R^{\prime \prime}$ pour , $\hat{x}_{k}\left\{\left(E x_{1}, \ldots x_{k-1}, x_{k+1}, \ldots x_{n}\right) R\left(x_{1}, \ldots x_{k}, \ldots\right.\right.$ $\left.\left.\ldots x_{n}\right)\right\}^{\prime \prime}$.

23.43. Si $R$ a $n$ termes, , $C^{\prime} R^{\prime \prime}$ pour , $D_{1}^{\prime} R \cup D_{2}^{\prime} R \cup \ldots D_{n}{ }^{\prime} R^{\prime \prime}$.

23.44. "Rトk $\alpha$ " pour , $\hat{x}_{1}, \ldots \hat{x}_{n}\left\{x_{k \varepsilon \alpha} \cdot R\left(x_{1}, \ldots x_{n}\right)\right\} "$.

23.45. "R\a" pour , $, \hat{x}_{1}, \ldots \hat{x}_{n}\left\{x_{1}, \ldots x_{n} \varepsilon \alpha \cdot R\left(x_{1}, \ldots x_{n}\right) "\right.$.

\subsection{Relations partielles.}

23.51. Chaque relation à $n$ termes contient $\left(\begin{array}{l}n \\ m\end{array}\right)$ relations partielles à $m$ termes.

Explication: $\left(\begin{array}{c}n \\ m\end{array}\right)=\frac{n \cdot(n-1) \cdot(n-2 \ldots \ldots(n-(m-1))}{m !}$ C'est le même théorème qui sert à calculer les coefficients du binôme de Newton. Pour $n$ et $m$ de 1 à 10 on obtient le tableau suivant:

23.52. Nombre de relations partielles:

\begin{tabular}{|c|c|r|r|r|r|r|r|r|r|}
\hline$n=$ & $m=2$ & 3 & 4 & 5 & 6 & 7 & 8 & 9 & 10 \\
\hline \hline 2 & 1 & & & & & & & & \\
3 & 3 & 1 & & & & & & & \\
4 & 6 & 4 & 1 & & & & & & \\
5 & 10 & 10 & 5 & 1 & & & & & \\
6 & 15 & 20 & 15 & 6 & 1 & & & & \\
7 & 21 & 35 & 35 & 21 & 7 & 1 & & & \\
8 & 28 & 56 & 70 & 56 & 28 & 8 & 1 & & \\
9 & 36 & 84 & 126 & 126 & 84 & 36 & 9 & 1 & \\
10 & 45 & 120 & 210 & 252 & 120 & 120 & 45 & 10 & 1 \\
\hline
\end{tabular}

72 
Exemple: une relation à 4 termes $R(x, y, z, t)$ contient 6 relations partielles binaires (entre $x-y, x-z, x-t, y-z, y-t$ et $z-t$ ), 4 relations ternaires (entre $x-y-z, x-y-t, x-z-t$, et $y-z-t)$ et une relation quaternaire $(R)$. Toutes ces relations ont, en plus, leurs converses.

23.53. , , $\left(R_{n}^{m}\right)$ " pour ,l'n-tiéme relation partielle à $m$ termes contenue dans $R^{\prime \prime}$.

Exemple: , $\left(R_{2}^{3}\right)$ " pour ,la deuxième relation ternaire contenue dans $R$.

LitTÉRAture: Carnap, Abriss. 


\section{V \\ DIVERS}

$\S 24$. Forme CANONIQUe Outre la méthode exposée au $\S 4$, il en existe encore une autre, dite ,méthode de la forme canonique" ou "normale". Son exposé ne pouvait être donné avant la construction de la théorie des règles (§9); aussi a-t-il été renvoyé au présent chapitre. C'est d'une manière sommaire et privée de rigueur que nous présentons ici cette théorie.

24.11. „Forme canonique" pour: „produit logique dont chaque argument est une somme logique de variables ou de négations de variables".

Exemple: „( $(p \vee \sim q) \cdot(p \vee q) \cdot(\sim p \vee r)$ " est une forme canonique.

24.12. Toute proposition du système 8 peut être transformée en forme canonique équivalente dont chaque argument contient des variables isomorphes à toutes les variables de la proposition. Cette transformation s'accomplit en vertu des règles qui correspondent (par le procédé du §9) aux lois associatives et distributives de la somme et du produit (5.23-4,5.53-4), au principe de la double négation (5.12), aux lois de de Morgan $(5.27,5.57)$, et aux lois 5.311 et 5.612.

Explication: en pratique, ceci veut dire qu'on doit „multiplier" avec ..v" et „..", comme en algèbre, substituer „ $\sim p "$ par , $p ", \ldots \sim . p \vee q "$ par,$\sim p . \sim q "$, $" \sim . p q "$ par $, \sim p \vee \sim q ",{ }, p \supset q "$ par,$\sim p \vee q "$ et,$p \equiv q "$ par , $p \supset q . q \supset p "$, en répétant ces opérations aussi longtemps qu'il est nécessaire pour obtenir la forme canonique. - Il est conseillé d'employer l'écriture péano-russéllienne avec des parenthèses, à cause de la ressemblance de cette écriture à celle de l'algèbre, ce qui facilite la "multiplication".

Exemple: soit à transformer la proposition:,$(p \supset q) \supset(\sim q \supset \sim p)$ ". En appliquant 5.311 nous obtenons d'abord .. $(\sim p \vee q) \supset(q \vee \sim p) "$, puis, par une seconde application de la même règle: $\sim \sim(\sim p \vee q) \vee(q \vee \sim p) "$. La loi de de Morgan donne: ,, $(p . \sim q) \vee(q \vee \sim p)$ ". De là, en "multipliant", on obtient: " $(p \vee q \vee \sim p)(\sim q \vee q \vee \sim p) "$. Cette dernière formule est la forme canonique.

24.13. Règle: la forme canonique est une loi alors et alors seulement quand chaque argument du produit contient au moins une variable avec son isomorphe précédée d'une négation.

Explication: en vertu de la règle fondée sur $6.13, \ldots p \vee \sim p$ " est toujours vrai; d'autre part, en vertu de 6.26, si un argument quelconque de l'alternative est vrai, l'ensemble l'est aussi. Enfin le produit de propositions vraies est vrai lui-même (cf. 4.23). — La règle 24.3 permet donc d'évaluer une proposition. 
LitTÉRATURE: Hilbert-Ackermann, Grundzüge; Quine, Logic of quantification (application aux expressions quantifiẻes).

\section{$\S 25$. LOGIQUES POLYVALENTES ET COMBINATOIRES; MÉTA- LOGIQUE FORMALISÉE}

Ce chapitre contient de brèves indications sur trois domaines où les recherches logiques se sont récemment engagées. Ces domaines n'ont du reste rien de commun.

Les logiques polyvalentes sont encore assez discutées; les combinatoires le sont moins, cela, semble-t-il, parce qu'elles sont peu connues; la métalogique formalisée est, par contre, une discipline déjà bien établie.

\subsection{Logiques multivalentes.}

En admettant deux valeurs seulement (3.11), on forme une logique bivalente; si les valeurs admises sont au nombre de trois $\left(, 1 ",,, \frac{1}{2},,, 0^{\prime \prime}\right.$, ou , 1", ,2", ,"3"), on obtient une logique trivalente; en généralisant, $n$ valeurs admises (où , $n$ " est substitué par un nombre entier positif) donnent une logique à $n$ valeurs. Le nombre d'opérateurs de vérité $n$-adiques dans une logique à $m$ valeurs est $m^{m^{n}}$ on a donc pour une logique à

\begin{tabular}{rrcl}
2 & 3 & 4 & valeurs \\
\hline 4 & 27 & 256 & opérateurs monadiques et \\
16 & 19,683 & $4,294,967,296$ & opérateurs dyadiques
\end{tabular}

Il est donc possible de définir dans des logiques à plus de deux valeurs beaucoup d'opérateurs intraduisibles en termes de la logique bivalente, comme par ex. les opérateurs de la modalité. D'autre part certaines lois de la logique bivalente ne sont pas des lois de la logique trivalente ou à nombre supérieur de valeurs. Par exemple, le principe du tiers exclu n'est pas valable en logique trivalente, car, en substituant $p / \frac{1}{2}$ et $q / \frac{1}{2}$ dans cette loi, on obtient,$\frac{1}{2} \vee \frac{1}{2} "$, ce qui, d'après la définition de

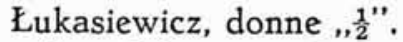

Les définitions des opérateurs principaux sont les suivants, d'après Łukasiewicz:

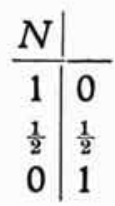

\begin{tabular}{c|ccc}
$A$ & 1 & $\frac{1}{2}$ & 0 \\
\hline 1 & 1 & 1 & 1 \\
$\frac{1}{2}$ & 1 & $\frac{1}{2}$ & $\frac{1}{2}$ \\
0 & 1 & $\frac{1}{2}$ & 0
\end{tabular}

\begin{tabular}{c|ccc}
$C$ & 1 & $\frac{1}{2}$ & 0 \\
\hline 1 & 1 & $\frac{1}{2}$ & 0 \\
$\frac{1}{2}$ & 1 & 1 & $\frac{1}{2}$ \\
0 & 1 & 1 & 1
\end{tabular}

\begin{tabular}{c|ccc}
$K$ & 1 & $\frac{1}{2}$ & 0 \\
\hline 1 & 1 & $\frac{1}{2}$ & 0 \\
$\frac{1}{2}$ & $\frac{1}{2}$ & $\frac{1}{2}$ & 0 \\
0 & 0 & 0 & 0
\end{tabular}

mais d'autres définitions sont possibles.

\subsection{Logique combinatoire.}

La logique combinatoire est la théorie des opérateurs dits „,om- 
binateurs" qui indiquent une opération formelle à effectuer sur n'importe quel type d'expression. Les principaux combinateurs sonts les suivants: le combinateur ",$B$ " qui porte le nom de ,compositeur élémentaire", transforme une expression composée de 3 termes, en groupant le 2 e et le $3 e$ dans une parenthèse. „CC”, dit „,permutateur élémentaire”, transforme une expression composée de 3 termes, en intervertissant l'ordre du $2 e$ et du $3 e$ terme. "I", appelé „Identificateur élémentaire”, transforme un terme en lui-même. „ $W$ ", rendu par „,répétiteur élémentaire", transforme une expression composée de deux termes, en doublant le deuxième de ces termes.

L'application de ces combinateurs ne se fait pas directement aux expressions de la logique mathématique classique, mais à des formules dans lesquelles apparait l'opérateur lambda $\left(, \lambda^{\prime \prime}\right)$. Cet opérateur joue un rôle semblable à celui des variables à accent circonflexe $\left(, \hat{x}^{\prime \prime}\right)$, (15.111), mais généralisé. L'expression , , $\alpha[M]$ " représente p. ex. l'opération qui, appliquée à , $\alpha "$, la transforme en l'expression ",M".

Les combinateurs peuvent être combinés entre eux, donnant par là de très grandes possibilités de simplification et de généralisation de la logique.

\subsection{Métalogique formalisée.}

La métalogique, dite aussi „sémiotique” (cf. 2.16) comporte trois parties: (1) la syntaxe logique (théorie des relations entre les symboles), (2) la sémantique (théorie des relations entre les symboles et ce qu'ils signifient), (3) la pragmatique (théorie des relations entre les symboles et ceux qui les emploient). Les deux premières peuvent être, à leur tour, formulées en termes conventionnels et formalisées (7.51). Ce procédé permet d'élaborer très exactement les notions logiques, d'axiomatiser la métalogique, d'étudier d'une manière rigoureuse le caractère complet et non-contradictoire des systèmes (7.6), l'indépendance des axiomes (7.7), de définir avec précision les termes métalogiques constamment utilisés en logique, tels que „système”, ",déduction", ,terme", „,variable”, ,expression" etc. Les systèmes sont alors traités comme classes.

Le procédé technique consiste généralement à donner aux termes du système examiné une double traduction, comme on l'a fait au $\S 9$. A chaque terme est coordonné un signe métalogique; le fait que le terme $Y$ suit le terme $X$ est exprimé par un symbole spécial.

La métalogique s'est avérée très féconde en conclusions philosophi- 
ques, notamment quant à la définition de termes tels que "vérité", "signification" etc.

Histolre: Les logiques multivalentes sont une découverte de Eukasiewicz (1920) et Post (1921). Elles furent surtout cultivées en Pologne, où Wajsberg donna (1931) un premier système axiomatique. On a beaucoup discuté plus récemment sur la relation entre ces logiques et le calcul de probabilité, comme aussi sur les implications philosophiques. - La logique combinatoire, qui est la branche la plus récente de notre science, fut inaugurée par Schönfinkel en 1924 et développée par Curry et Kleene; Church donna en 1936 une synthẻse perfectionnée des travaux antérieurs. - La métalogique (dite aussi ,sémantique") a ses précurseurs dans la théorie de la supposition et dans les traités „de modis significandi" médiévaux, mais comme discipline exacte elle est très récente. La "métamathématique" de Hilbert (1905), les spéculations sur le langage du cercle de Vienne (1929 ss.) et l'axiomatisation rigoureuse des systèmes polonais ont été les trois sources de la nouvelle discipline Son développement est dû surtout aux travaux de Carnap, Gödel, Leśniewski et Tarski.

LitTÉRATURE: § 25.1: Feys, Les logiques (avec bibliographie); § 25.2; Feys, La technique; $\S 25.3$ : Carnap, Foundations; Introduction; Tarski, Wahrheitsbegriff; bon résumé dans Quine, Mathematical Logic.

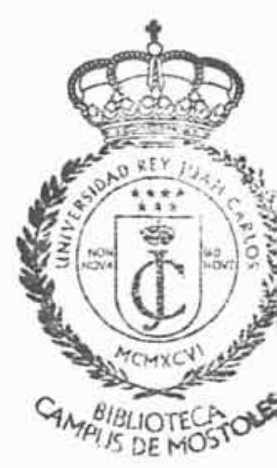




\section{BIBLIOGRAPHIE}

Abréviations: „JSL" pour: "The Journal of symbolic Logic".
"PM" pour: "Principia mathematica" (cf. Whitehead)

ACKermann, W., Ein System der Typenfreien Logik, I, Leipzig 1941. Cf. Hilbert.

AjDUkıEwicz, K., Zalożenia logiki tradycyjnej (= Les présupposées de la logique traditionelle), Przegl. Filozoficzny 29, 1926/7.

Die Syntaktische Konnexität, Studia Philosophica (Lwów) I, 1935.
Ueber die Anwendbarkeit der reinen Logik auf philosophische Probleme, Actes du VIIe Congr. Int. de Philos., Prague 1936.

Aristoteles, Organum, éd. Th. Waitz, Leipzig 1844-1846.

Becker, A., Die Aristotelische Theorie der Möglichkeitsschlüsse, Berlin 1933.

Behmann, H., $\mathrm{Zu}$ den Widersprüchen der Logik und der Mengenlehre, Jahresberichte d. Math. Ver., 40, 1931.

Bennett, A. A. and BAylis, CH. A., Formal Logic, New York 1939.

BERKEley, E. C., Conditions affecting the application of symbolic Logic, JSL 7, 1942.

Bernays, E. C., A System of axiomatic Set Theory. JSL 2, 1937; 6, 1941; 7, 1942 ;

8, 1943.

Cf. Hilbert.

BETH, E. W., Inleiding tot de wijsbegeerte der wiskunde, Antwerp, Brussels, Nijmegen, Utrecht 1940; 2e éd. 1948.

- Summulae Logicales, Groningen 1942.

- Geschiedenis der Logica, Den Haag 1944.

Mathematische Logik und Grundlegung der exakten Wissenschaiten (I. M. Bochenski (ed.): Bibl. Einführungen i. d. Studium der Philos., N. 3), 1948.

BLACK, M., The Nature of Mathematics, New York 1934.

- A new Method of Presentation of the Theory of the Syllogism, Journal of Philosophy, 1945.

BOCHENSKI, I. M., Notes historiques sur les propositions modales, Revue des Sciences Philos. et Théol. 26, 1937.

- De consequentiis Scholasticorum earumque origine, Angelicum 15, 1938.

- Nove Lezioni di Logica Simbolica, Roma 1938.

On the Categorical Syllogism, Dominican Studies 1, 1948.

Boll, M., Manuel de logique scientifique, Paris 1948.

Boole, G., An Investigation of the Laws of thought, London 1854 (réimpr. dans: Colected Logical Works, Chicago and London 1916.

The mathematical Analysis of Logic, Cambridge 1847 (réimpr. ibid. et Oxford 1948).

CARnAP, R., Abriss der Logistik, Wien 1929.

The Logical Syntax of Language, London and New York 1937.

- Foundations of Logic and Mathematics, Intern. Encyclopedia of unified Science, I, 3, Chicago 1939.

Introduction to Semantics, Cambridge Mass. 1942, 3 ed. 1948. 
Formalization of Logic, Cambridge Mass. 1943.

Church, A., A Bibliography of Symbolic Logic, JSL 1, 1936.

A Formulation of the simple Theory of Types, JSL 5, 1940.

Chwistek, L., Antynomie logiki formalnej, Przegl. Filozoficzny, 24, 1921.

The Théory of constructive Types, Ann. de la Soc. Polonaise des máthémat. 2, 1924.

New Foundations of formal Metamathematics, JSL 3, 1938.

Cooley, J. C., A Primer of Formal Logic, New York 1942.

Couturat, L., La logique de Leibniz d'après des documents inédits, Paris 1901.

CURRY, H. B., Grundlagen der kombinatorischen Logik, Amer. Journ. of Math. 52, 1930.

- On the Use of Dots as Brackets in Logical Expressions, JSL 2, 1937.

DiEUdonNE, J., Les méthodes axiomatiques modernes et les fondements des mathématiques, Rev. Scientifique 77, 1939.

DüRR, K., Aussagenlogik im Mittelalter, Erkenntnis 7, 1938.

FEYS, R., La transcription logistique du raisonnement, Revue Néoscolastique de Philosophie, 26-27, 1924-1925.

- Le raisonnement en termes de faits dans la logique russellienne, ib. 29-30, 1927-8.

- Les logiques nouvelles de la modalité, ib. 40-41, 1937-8.

- Directions nouvelles de la logistique aux Etats-Unis, ib. 44, 1946.

Logistiek, I, Antwerpen-Nijmegen 1944.

Les méthodes récentes de déduction naturelle, Rev. Philos. de Louvain, 44, 1946.

Fitch, F. B., A System of Formal Logic without an analogue to the Curry $W$ Operator, JSL I, 1936.

FRAENKEL, A. et BAR-HILleL, J., Le problème des antinomies et ses développements récents, Revue de métaphysique et de morale 46, 1939.

FREGE, G., Begriffschrift, Halle 1879.

— Grundlagen der Arithmetik, Breslau, 1884, 2ème éd. 1934.

Grundgesetze der Arithmetik, Jena, I, 1893, II, 1903.

Gentzen, G., Untersuchungen über das logische Schliessen, Math. Zeitschr. 39, 1934.

GöDEL, K., Uuber formal unentscheidbare Sätze der Principia Mathematica und verwandter Systeme I, Monatshefte Math. Phys. 38, 1930.

Gonseth, F., Les fondements des mathématiques, Paris 1926.

- Qu'est-ce que la logique? Paris 1937.

(ed) Les entretiens de Zürich sur les fondements et la méthode des sciences mathématiques, Zürich, 1941.

Greenwood, Th., Les fondements de la Logique symbolique (2 fasc.), Paris, 1938.

HENLE, P., The Status of Emergence, Journal of Philos. 39, 1942.

Heyting, A., Die formalen Regeln der intuitionistischen Logik, Stzb. d. Preuss.

Ak. d. Wiss., Phys.-Math. Kl. 1930.
Mathematische Grundlagenforschung. Intuitionismus. Beweistheorie, Berlın 1934.

Hilbert, D. und AckermanN, W., Grundzüge der theoretischen Logik, Berlin, 1928, 2ème éd. 1938.

Hilbert, D. und Bernays, P., Grundlagen der Mathematik, Berlin, I, 1934, II, 1939; 2e éd. Michigan 1944. 
Huntington, E. V., Sets of independent Postulates for the Algebra of Logic, Trans. Amer. Math. Soc., 5, 1904.

HUSSERL, E., Logische Untersuchungen, Halle, I, 1900, II, 1901.

JAśKOWSKI, S., On the rules of suppositions in Formal Logic, Warsaw 1934.

Joergensen, J., A treatise of Formal Logic (3 vol.), London 1931.

JoRDAN, Z., The Development of Mathematical Logic and of Logical Positivism in Poland., London 1945.

Keynes, J. N., Studies and Exercices in Formal Logic, London 1894, 4e éd. 1906.

KleENE, S. C., A theory of positive Integers in Formal Logic, Amer. Journal of Mathem., 57, 1935.

KURATOWSKI, K., Sur la notion de l'ordre dans la théorie des ensembles, Fundamenta Mathematicae 2, 1921.

LESNIEWSKI, ST., Lleber die Grundlagen der Ontologie, C. r. de la Soc. des Sc. et des Lettres de Varsovie, 61. III, 1930.

- Grundzüge eines neuen Systems der Grunlagen der Mathematik, § 12, Warschau 1938.

- Einleitende Bemerkingen zur Fortsetzung meiner Mitteilung u. d. T. „Grundzüge......", Warschau, 1938.

LEWIS, C. I., A survey of Symbolic Logic, Berkeley 1918.

Lewis, C. I. and LANGFord, C. H., Symbolic Logic, New York 1936.

ŁUKASIEWICZ, J., O logice trójwartościowej (= Sur la logique trivalente), Ruch Filozoficzny, 5, 1920.

- Logika dwuwartosciowa (= Logique bivalente), Przeglad Filozoficzny, 23, 1931.

- Elementy logiki matematycznej, Warszawa 1929.

Philosophische Bemerkungen zu mehwertigen Systemen des Aussagenkalkuels, C. r. Soc. d. Sciences et des Lettres Varsovie Cl. III, 23, 1930.

- Zur Geschichte der Aussagenlogik, Erkenntnis 5, 1935/6.

Die Logik und das Grundlagenproblem, dans: F. GoNSETH, Entretiens, 1941.

ŁUKASIEIVICZ, J. und TARSKI, A., Untersuchungen ueber den Aussagenkalkuel, C. r. Soc. d. Sciences et des Lettres Varsovie, Cl. III, 23, 1930.

Menger, K., Moral, Wille und Weltgestalltung. Grundlegung der Logik der Sitten, Wien 1934.

Miller, J. W., The Structure of Aristotelian Logic, London 1938.

MoISIL, G. C., Recherches sur l'algèbre de la logique, Ann. scientifiques de l'Université de Jassy 22, 1936.

- Recherches sur le syllogisme, ibid. 25, 1939.

MOORE, G. E., Russell's „Theory of Descriptions", dans: The Philosophy of Bertrand Russell, ed. P. A. SCHILPP, Evanston et Chicago 1944.

Morgan, A. DE, Formal Logic, London 1847; 2e éd. London 1926.

- On the Syllogism No IV, and on the Logic of Relations, Trans. Cambr. Philos. Soc. 10, 1864.

NiCOD, J., A Reduction in the number of primitive propositions of Logic, Proc. Cambr. Philos. Soc., 19, 1917/20.

Oppenheim, F. E., Outline of a logical Analysis of Law, Philosophy of Science 11, 1944. 
PeANo, G., Formulaire mathématique, I, Turin, 1895; II, 1, Turin, 1897; II, 2, Turin, 1898; II, 3, Turin, 1889; III, Paris, 1901; IV, Turin, 19002-3.

Formulario matematico, V, Torino 1905-8.

PEIRCE, C. S., Collected Papers, éd. C. Hartshorn and P. Weiss, Cambridge Mass. 1931-6 (6 vol.).

POPPeR, K. R., New Foundations for Logic, Mind 56, 1947.

PosT, E. L., Introduction to a general theory of propositions, Amer. Journ. of Mathem. 43, 1921.

Quine, W. v. O., A System of Logistic, Cambridge, 1934.

- On the Theory of Types, JSL 3, 1938.

- Mathematical Logic, New York 1940, 2 éd. 1947.

- On the Logic of Quantification, JSL 10, 1945.

Ramsey, F. P., The Foundations of Mathematics, Proc. Lond. Mathem. Soc. 25, 1926.

Russell, B. A. W., The Principles of Mathematics, I., Cambridge 1903; 2e éd. 1938.

- On denoting, Mind 14, 1905.

Cf. WhiteHEAD.

Introduction à la philosophie mathématique, tr. de G. Moreau, Paris 1928.

RÜstow, A., Der Lügner. Theorie, Geschichte und Auflösung, (Diss. Erlangen), Leipzig 1910.

SALAMUCHA, J., Pojawienie się zagadnien antynomialnych na gruncie logiki średniowiecznej (= Apparition des problèmes des paradoxes dans la logique médièvale), Przeglad Filozoficzny 40, 1937.

SCHÖNFINKEl, M., Ueber die Bausteine der mathematischen Logik, Math. Annalen 42, 1925.

Scholz, H., Geschichte der Logik, Berlin 1931.

- Metaphysik als strenge Wissenschaft, Köln 1941.

SChroeder, E., Vorlesungen ueber die Algebra der Logik, Leipzig, I, 1890, II, 1, 1891; II, 2, 1905; III, 1895.

SChroeter, K., Ein allgemeiner Kalkuelbegriff, Leipzig 1941.

- Axiomatisierung der Fregschen Aussagenkalküle, Leipzig 1943.

Serrus, CH., Traité de Logique, Paris 1945.

SoBocınSKı, B., Aksjomatyzacja implikacyjno-konjunkcyjnej teorii dedukcji, Przegl. Filozoficzny, 38, 1935.

- Aksjomatyzacja pewnych wielowartościowych systemów teorii deduckji ( = Axiomatisation de certains systèmes polyvalents de la théorie de la déduction), Roczniki Zrzesz. asyst. Uniw. J. P., Warszawa 1936.

SтAмm, E., Zastosowanie algebry logiki do teorii szyfröw (= Application de l'algébre de la logique à la théorie des chriffres), Rozpr. Pol. Tow. Matem. I, 1921.

Stebing, S., A modern Introduction to Logic, New York 1931.

A modern elementary Logic, London 1943.

TARSKI, A., Fundamentale Begriffe der Methodologie der deduktiven Wissenschaften, Monatshefte f. Mathem. u. Physik 37, 1930.

- Der Wahrheitsbegriff in den formalisierten Sprachen, Studia Philosophica (Lwów) I, 1935.

Grundzüge des Systemenkalkuels, I, Fundamenta Mathematicae, 25-26, 1935-1936. 
On the Calculus of Relations, JSL 6, 1941.

Introduction to Logic and to Methodology of deductive Sciences, New York 1941.

ThомAs, I., Logic and Theology, Dominican Studies 1, 1948.

Turing, A. M., The use of Dots as Brackets in Church's System, JSL 7, 1942.

Ushenko, A. P., The Problems of Logic, London 1941.

WAJSBERG, M., Aksjomatyzacja trójwartściowego rachunku zdan் (= axiomatization du calcul trivalent des propositions), C. r. Soc. Sciences et Lettres de Varsovie 24, 1931.

Whitehead, A. N. and Russell, B., Principia Mathematica, Cambridge, I, 1910; II, 1912; III, 1913; 2e éd.: I, 1925, II-III, 1927.

Wiener, N., A Simplification of the Logic of Relations, Proc. Cambr. Philos. Soc. 17, 1912/14.

Woodger, J. H., The axiomatic Method in Biology, Cambridge 1937 (avec appendice par TARSKI, A.).

The Formulation of a psychological Theory, Erkenntnis 7, 1937.

The Technique of Theory Construction, Intern Encyclop. of Unified Science II, 5, Chicago 1939.

ZERMELO, E., Untersuchungen ueber die Grundlagen der Mengenlehre, Mathem. Annalen 65, 1908. 


\begin{tabular}{|c|c|c|c|c|c|}
\hline 2.3 & ()$,[],\{\}$ & 15.13 & $\varepsilon$ & 19.31 & $\check{R}$ \\
\hline 2.4 & $\cdot:,::,::$ & 15.14 & $\sim \varepsilon$ & 19.32 & Cnv \\
\hline 3.11 & 1,0 & 15.21 & - & 20.11 & $D^{\prime} R$ \\
\hline 3.12 & $=$ & 15.22 & U & 20.12 & $Q^{\prime} R$ \\
\hline 3.23 & $\sim, N$ & 15.23 & ก & 20.13 & $C^{\prime} R$ \\
\hline 3.41 & v, $A$ & 15.24 & $\|$ & 20.31 & $a \uparrow R$ \\
\hline 3.51 & $\supset, C$ & 15.25 & c. & 20.32 & $R+\beta$ \\
\hline 3.61 & I, D & 15.26 & $=$ & 20.33 & $\alpha 1 R \uparrow \beta$ \\
\hline 3.71 & $\cdot K$ & 15.41 & v & 20.34 & $R\lceil\alpha$ \\
\hline 3.81 & $\equiv, E$ & 15.42 & $\wedge$ & 20.35 & $\uparrow$ \\
\hline 3.93. & $\bar{p}, \rightarrow, \mathcal{E}, \sim$ & 15.43 & G! & 20.37 & $\downarrow$ \\
\hline 8.111 & $p, q, r, s$ & 15.61 & {$[x]$} & 20.41 & $1 \rightarrow \mathrm{Cls}$ \\
\hline 9.51 & $+, \times, \vdash, \frac{X}{Y}$ & 15.62 & {$[x, y]$} & 20.42 & $\mathrm{Cls} \rightarrow 1$ \\
\hline $10.001 b$ & $b a, b, m^{Y}$ & 15.63 & 1 & 20.43 & $1 \rightarrow 1$ \\
\hline $10.001 c$ & $\begin{array}{l}D a, b, m \\
C A, I\end{array}$ & 15.64 & 2 & 21.11 & $R \mid S$ \\
\hline 10.01 & $E$ & 18.11 & $\hat{x} \hat{y}\{f(x, y)\}$ & 21.12 & $R^{2}$ \\
\hline 10.02 & O & 18.12 & Rel & 21.13 & $R^{3}$ \\
\hline 11.11 & $a, b, c, d$ & 18.14 & $x R y$ & 21.14 & $R^{n}$ \\
\hline 11.12 & $x, y, z, t$ & 18.21 & $\dot{-}$ & 21.15 & $R^{\circ}$ \\
\hline 11.13 & $f, g, h, i, j$ & 18.22 & U் & 21.21 & her \\
\hline 11.16 & $f x$ & 18.23 & ก் & 21.22 & $R_{*}$ \\
\hline 11.21 & $(x), \Pi x$ & 18.24 & $\|$ & 21.23 & $R_{p o}$ \\
\hline 11.22 & $(E x), \Sigma x$ & 18.25 & c. & 21.31 & $B$ \\
\hline 13.11 & $f(x, y)$ & 18.26 & $\stackrel{\circ}{=}$ & 21.32 & $\operatorname{Min}_{R}$ \\
\hline 13.12 & $(x, y), \Pi x y$ & 18.27 & $\overline{\bar{v}}$ & 21.33 & $\operatorname{Max}_{R}$ \\
\hline 13.13. & $(E x, y), \Sigma x y$ & 18.28 & $\dot{i}$ & 21.41 & $R+\stackrel{R}{S}$ \\
\hline 14.11 & $=$ & & & 21.42 & $\overline{s m o t}$ \\
\hline 14.12 & $\neq$ & 18.29 & 白! & 21.43 & Smor \\
\hline 14.16 & I & 19.11 & $R^{\prime} y$ & 22.11 & $r e f$ \\
\hline 14.17 & $J$ & 19.12 & $\vec{R}^{\prime} y$ & 22.12 & itr \\
\hline 14.22 & $(7 x)(f x)$ & 19.12 & & 22.21 & sym \\
\hline 14.23 & $E !$ & 19.13 & $R^{\prime} x$ & 22.22 & as \\
\hline 15.11 & $\hat{x}$ & 19.14 & $s g^{\prime} R$ & 22.31 & trans \\
\hline 15.111 & $\lambda$ & 19.15 & $g s^{\prime} R$ & 22.32 & intr \\
\hline 15.12 & $C l s, \alpha, \beta, \gamma$ & 19.21 & $R^{\prime \prime} \beta$ & 22.41 & sim \\
\hline
\end{tabular}




$\begin{array}{ll}22.42 & \text { aeq } \\ 22.51 & \text { connex } \\ 22.52 & \text { ser } \\ 23.12 & \text { Rel } l_{n} \\ 23.22 & R_{k}\end{array}$

$23.24 \vec{R}_{k}$,

$23.25 \mathrm{sg}_{k}{ }^{\prime} R$

$23.32 R^{(a \ldots \ldots \ldots u)}$

$23.42 D_{k}^{\prime} R$

$23.44 R \uparrow_{k} \alpha$
$23.45 R\lceil\alpha$

$23.53\left(R_{n}^{m}\right)$

$25.1 \quad A, C, K, N$

$25.2 B, C, I, W$

$25.2 \lambda$ 


\section{TABLE DES TERMES TECHNIQUES FRANÇAIS}

Absurde (lois de la réduction à $1^{\prime}$ ) $6.23-5 ; 6.68$

Addition (loi) 6.63

A fortiori (loi) 6.27

Alternative $3.4 ; 9.22$

Analogie (principe d') 17.6

Analogues (lois) $12.5 ; 13.3 ; 16.1 ; 18.3 ; 23.16$

Ancestrale (relation) 21.2

Antécédent relatif 18.15

Argument 1.35

Associative (loi) $5.23 ; 5.53 ; 5.63$

Assymétrie 22.22; 22.24

Axiome 7.43; indépendance 7.73

Calcul des classes 16

Carré logique (lois du) 10.2

Catégorie sémantique 1.22

Champs 20.13; 23.43; (lois) 20.2

Classes 16; calcul des 16; binaire 15.62; complémentaire 16.21; relations entre 15.2; unitaire 15.61 ; universelle 15.41 ; vide 15.42

Clôture 11.36-8

Co-domaine 20.12; (lois) 20.2

Composition (lois) 6.61-3

Commutation (loi) 5.33

Conjonction 3.7; 9.26

Connexité 22.5

Conséquent relatif 18.16

Consistance 7.6

Constante 1.13; nominale 11.11

Contraposition (lois) 5.32-322; 5.37-8

Converse 19.3-4; 23.3

Conversion (lois) 10.2

Corners 2.13

Déduire 7.42

Déduction (règles) 7.45; 8.3; (systèmes de règles) 9

Définition 1.5, 7.22; (règle de la) $7.25 ; 8.12$

Dérivation (règle) 8.33

Description 14.2; relative 19, 23.2; individuelle 19.11, 23.21-2; plurielle 19.12-15. 23.23-25; bi-plurielle 19.2; multiplurielle 23.26

Dilemme (lois) 6.64-71

Disjonction 3.6; 9.24; des classes 15.24

Distributives (lois) $5.24 ; 5.54$

Domaine 20.11, 23.41-2; lois 20.2

Ecriture (règles) 2

Egalité (des classes) 15.26; (des relations) 18.26 


\section{Enchaînement relatif 21}

Equivalence 3.8; 9.25

Equivalences 5

Equivocité 17.63

Evaluation 4; 4.12; (technique) 4.2

Existence 14.23, 15.43, 18.29; (lois) 16.4

Exportation (lois) $5.34-5$

Expression 1.11-2; 7.3; élémentaire 4.13

Extensionnalité (thèse) 14.11

Facteur nouveau (loi) 6.26

Fonction propositionnelle 1.32

Formalisme 7.5

Formation (règles) $7.31-2$

Gonseth (schèmes) 3.9

Hilbert-Ackermann (système) 8

Identité (principe) $5.11 ; 12.53 ; 14.13$; (théorie) 14.1

Image 21.41

Implication: formelle 11.39; inférentielle 7.48; matérielle 3.5

Inclusion 15.25; des relations 18.25

Indiscernables (principe) 14.11

Inversion (règle) 5.72

Isomorphes: termes 1.15 ; relations 21.4

Isomorphie 21.4

Lambda (opérateur) 15.111; 18.111; 25.2

Loi logique $4.11 ; 7.41$

Łukasiewicz: écriture 2.21-2; méthode 8; syllogistique 10

Matrice 11.15

Modes syllogistiques 10.5

Modus: ponendo ponens $6.41-423$, ponendo tollens $6.53-543$; tollendo ponens $6.51-524$; tollendo tollens $6.43-443$

Métalogique 2.16

Métathéorie 2.15

de Morgan (lois): 1re 5.27; 2e 5.57; 3e 5.213; 4e 5.511

Mouvements des quantificateurs (lois) $12.6 ; 13.2$

Multiplication logique 3.7; (lois) $6.61-2$

Négation 3,2; 9.21; (principe de la double n.) 5.12; des prédicats quantifiés 12.2

Non- 9.21

Non-contradiction (principe) $6.11 ; 12.55$

Opérateur 1.34; de vérité 3 ; dyadiques 3.3 ; n-adiques 1.45 ; nominal $1.44 ; 11.13$; propositionnel 1.43

Paradoxales (lois) 6.21-2

Paradoxe de la classe des classes 17.2

Parenthèses 2.3

Péano-russellienne (écriture) 2.23

Permutation (loi) 5.22; 5.52; 5.62

Points $2.4 ; 15.27$

Prédicats monadiques 11; (lois) 12; dyadiques 13

Principes premiers 6.1 
Produit logique: des propositions 3.7; des classes 15.23; des relations 18.23; relatif 21.1 Proposition 1.31; individuelle 11.14

Puissances relatives $21.12-15$

Quantificateur 11.2; universel 11.21; existentiel 11.22

Quine (méthode) 17.5

Réduction à l'absurde (lois) 6.23-24; 6.68

Règles (système) 9; de traduction 9.3

Relation 18 ss.; ancestrale 21.22; assymétrique 22.22; bi-univoque 20.43; complémentaire 18.21; d'égalité 22.42; connexe 22.51; intransitive 22.32; irréflexive 22.14 ; isomorphe 21.4; partielle 23.5; propriétés 22; réciproque 20.42 ; relations entre relations 18.2 ; 23.15 ; réflexive 22.11 ; semblable 22.41 ; sériale 22.52 ; transitive 22.31; universelle 18.27 ; univoque 20.41 ; vide 18.28 .

Réflexivité 22.1

Similitude 22.4

Simplification (lois) 5.25-6; 5.55-6

Somme des propositions 3.4 ; des classes 15.22 ; des relations $18.22,23.15$

Substitution 1.2; de l'implication 5.73

Supposition 2.1

Syllogisme (lois) 6.3; aristotélicien 10; conditionnel 6.4; copulatif 6.53-543; disjonctif $6.51-524$

Syllogistique aristotélicienne 10

Système axiomatique $7 ; 7.11$; complet et indépendant 7.7 ; formalisé 7.51 ; non-contradictoire 7.61

Tautologie (lois) 5.15-16

Terme 7.21; dérivé 7.24 ; primitif 7.23 ; premier et dernier et 21.3 ; relatif 18.17

Thèse 7.24

Tiers exclu (principe) $6.13 ; 8.53-4 ; 12.56 ; 16.342$

Traditionnelle, terminologie 3.9

Transformation (règle) 5.7

Transitivité 22.3

Types (théorie) 17; (règle) 17.2

Unité cardinale 15.63

Univocité relative 20.4

Valeur 3.11; des expressions élémentaires 4.23

Variable 1.14; libre 11.31; liée 11.32; nominale 1.42; 11.12; propositionnelle 1.41

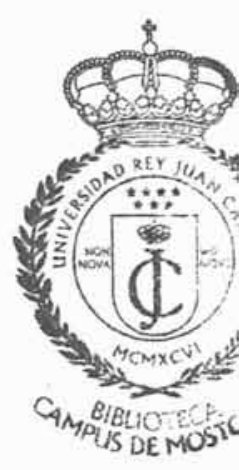




\section{TABLE DES MATIERES}

Préface

\section{GÉNÉRALITÉS}

\$ 0 . Introduction Page 0.1 . Notion et histoire. -0.2 Logique et mathématiques. -0.3 . Applications.

$\S 1$ Expressions et opérations fondamentales

1.1. Expression, constante, variable. - 1.2. Substitution, catégorie sémantique. - 1.3. Proposition, nom, opérateur. - 1.4. Division des variables et des opérateurs. - 1.5. Définition.

$\S 2$. Règles d'écriture

2.1. Suppositions. -2 2. Disposition des opérateurs. -2.3 . Parenthèses. - 2.4. Points.

\section{PROPOSITIONS}

§ 3. Opérateurs de vérité

3.1. Valeurs. - 3.2. Négation. - 3.3. Opérateurs dyadiques. -

3.4. Alternative ou somme logique. - 3.5. Implication matérielle. 3.6. Disjonction. - 3.7. Conjonction ou produit logique. - 3.8. Equivalence. - 3.9. Représentation graphique et terminologie.

§ 4. Evaluation

4.1. Définitions. - 4.2. Technique de l'évaluation.

§ 5. Equivalences

5.1. Lois avec toutes les variables isomorphes. -5.2 . Lois de la somme. - 5.5. Lois de l'implication. - 5.4. Lois de la disjonction. - 5.5. Lois du produit. - 5.6. Lois de l'équivalence. - 5.7. Règles de transformation.

$\S 6$. Premiers principes et implications . . . . . . . . . . 6.1. „Premiers principes”. - 6.2. Lois caractéristiques de l'implication. - 6.3. Loi du syllogisme. - 6.4. Modes du syllogisme conditionnel. 6.5. Modes du syllogisme disjonctif et copulatif. -6.6 . Lois de la composition et dilemmes.

§ 7. Système axiomatique

7.1. Définitions. - 7.2. Termes et définitions. - 7.3. Expressions et règles de formation. - 7.4. Lois et déduction. - 7.5. Formalisme. 7.6. Consistance. - 7.7. Système complet et indépendant. - 7.8. Règles.

§ 8. Système de la logique des propositions . . . . . . . . . . 30 8.1. Termes primitifs, règles et définitions. -8.2 . Axiomes. -8.3 . Déduction.

§ 9. Système des règles de la déduction

9.1. Définitions. - 9.2. Noms des expressions 8. - 9.3. Règles de traduction. - 9.4. Exemples des règles 9. - 9.5. Ecriture schématique et méthode de Gentzen. 


\section{PRÉDicats ET Classes}

Page

10.0. Termes primitifs et règles - . . . . . . . 37

10.2. Carré logique et con restes. - 10.1. Définitions et axiomes. -

§11. Prédicats monadiques conversion. - 10.5. Modes syllogistiques.

11.1. Définitions, -112 . . . . . . . . . . 41

§12. Lois des prédicats monatificateurs. - 11.3. Variables libres et liées.

12.1. Principe méthodique, -12.2 . Négation des prédicats monadiques quantifiés. - 12.3. Lois fondamentales. - 12.4. Règles de la déduction. -

12.5. Lois analogues. - 12.6. Lois du mouvement des quantificateurs. -

12.8. Lois syllogistiques. -12.9 . Lois does constantes.

§13. Prédicats dyadiques

13.1. Définitions. -13.2 . Lois du mouvement des quantificateurs. -
13.3. Lois analogues.

\$14. Identité et description

14.1. Identité. - 14.2. Descriptions.

\$15. Classes

15.1. Définitions fondamentales. - 15.2. Relations entre les classes. -

15.3. Représentation graphique. - 15.4. Existence. -15.5 . Lessens du mot „est". - 15.6. Classes unitaires et binaires.

§16. Calcul des classes

16.1. Lois analogues. -16.2 . Lois principales, -16.3 . Lois de la classe universelle et vide. -16.4 . Lois de l'existence.

§17. Théorie des types . . . . . . . . . .

17.1. Antinomies. - 17.2. Paradoxe de la classe des classes. - 17.3.

Règles des types. - 17.4. Méthodes de Quine. - 17.5. Principe d'analogie.

\section{Relations}

§18. Relations

18.1. Définitions. -18.2 . Relations entre relations. -18.3 . Lois analogues.

$\S 19$. Descriptions relatives. Inverse 19.1. Descriptions individuelles et plurielles. - 19.2. Descritpion bi-plurielle.

- 19.3. Inverse. - 19.4. Lois de l'inverse.

$\S 20$. Domaines et champs. Univocité relative

20.1. Domaines et champs. - 20.2. Lois des domaines et des champs. -

20.3. Relations aux domaines limités. - 20.4. Univocité relative.

§21. Enchainement relatif. Séries . . . . . . . . . . . .

21.1. Produits relatifs. -21.2 . Relation ancestrale. -21.3 . Premiers et derniers termes. - 21.4. Isomorphie des relations.

§22. Propriétés des relations . . . . . . . . . . . . .

22.1. Réflexivité. - 22.2. Symétrie. - 22.3. Transitivité. - 22.4 . Similitude. - 22.5. Connexité.

§23. Relations à plus de deux termes

23.1. Définitions fondamentales. -23.2 . Descriptions relatives. -23.3 . Converses. - 23.4. Domaines et champs. -23.5 . Relations partielles. 


\section{DiVers}

Page

§24. Forme canonique . . . . . . . . . . . . . . . . . 74

$\S 25$. Logiques multivalentes et combinatoires; métalogique formalisée . . 75 25.1. Logiques multivalentes. - 25.2. Logique combinatoire. -25.3 . Métalogique formalisée

Bibliographie . . . . . . . . . . . . . . . . 78

Table des expressions logiques . . . . . . . . . . . . . . . . . . 83

Table des termes techniques français . . . . . . . . . . . . 85

Table des matières . . . . . . . . . . . . . . . 88

N. obst.: N. Luyten, M.-D. Philippe.

Impr. p.: V. Kuiper. - Imprimatur: L. Waeber

13. XII . 1948. 


\title{
COLLECTION „SYNTHESE”
}

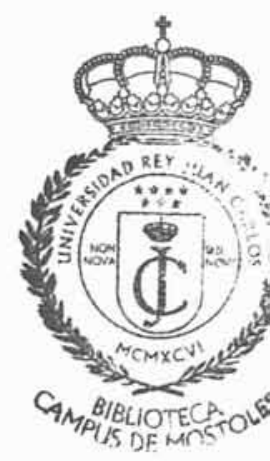

\section{Les fondements psycho-linguistiques des mathématiques}

\author{
par GERRIT MANNOURY \\ professeur émérite de l'Université d'Amsterdam
}

Un volume in $-8^{\circ}$, broché, Fl. holl. 4.90 , Fr. s. 6.60

Le Comité général de SYNTHESE et la Rédaction de DIALECTICA ont tenu à s'associer à l'hommage rendu à M. Mannoury à l'occasion de ses quatre-vingts ans, en faisant éditer une traduction française de l'étude que le savant hollandais a publiée en 1934 sur Die Signifischen Grundlagen der Mathematik.

La première partie de cet opuscule est une étude de la méthode psycholinguistique et des instruments intellectuels qu'elle met en oeuvre. Dans la seconde, l'auteur applique cette méthode aux sciences mathématiques et physique, dont il analyse certaines notions essentielles, comme la causalité, la probabilité, la vérité et la contradiction et montre qu'il est possible de déduire les mathématiques formalisées de systèmes d'expression plus généraux.

„L'étude que M. Mannoury a consacrée aux mathématiques constitue non seulement une analyse vivante et pleine d'esprit des notions fondamentales de cette science, mais aussi un acte de courage et de probité intellectuels, par ce refus constant de céder aux attraits des mythes qui entourent les sciences mathématiques."

A. Preissmann, dans Dialectica. 
